\title{
A Cell-Centered Lagrangian Scheme for Two-Dimensional Compressible Flow Problems
}

\author{
Rémi Abgrall ${ }^{1}$, Jérôme Breil ${ }^{2}$, Pierre-Henri Maire ${ }^{2}$, Jean Ovadia ${ }^{3}$ \\ (1) Institut Universitaire de France et Mathématiques Appliquées de Bordeaux, \\ Université Bordeaux I, 33405 Talence Cedex, France \\ (2) UMR CELIA CEA-CNRS-Université Bordeaux I, 33405 Talence Cedex, France \\ (3) CEA CESTA, 33114 Le Barp Cedex, France
}

July 25, 2006

\begin{abstract}
We present a new Lagrangian cell-centered scheme for two-dimensional compressible flows. The primary variables in this new scheme are cell-centered, i.e., density, momentum and total energy are defined by their mean values in the cells. The vertex velocities and the numerical fluxes through the cell interfaces are not computed independently contrary to standard approaches but are evaluated in a consistent manner due to an original solver located at the nodes. The main new features of the algorithm is the introduction of four pressures on each edge, two for each node on each side of the edge. This extra degree of freedom, allows us to construct a nodal solver which fulfills two properties. First, the conservation of momentum and total energy is ensured. Second, a semi-discrete entropy inequality is provided. In the case of a one-dimensional flow, the solver reduces to the classical Godunov acoustic solver: it can be considered as its two-dimensional generalization. Many numerical tests are presented. They are representative test cases for compressible flows and demonstrate the robustness and the accuracy of this new solver.
\end{abstract}

\section{Contents}

1 Introduction

2 Derivation of the Euler equations in the Lagrangian formalism 3

3 Spatial approximation $\quad \mathbf{6}$

3.1 Notations, assumptions and problem statement . . . . . . . . . . . . . . . . . 6

3.2 Approximation of the volume equation . . . . . . . . . . . . . . . . . 8

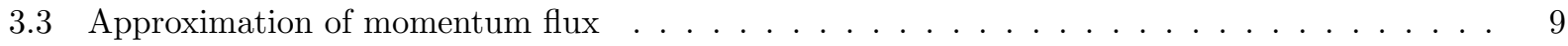

3.4 Approximation of the energy flux . . . . . . . . . . . . . . . . . . 10

3.5 Summary . . . . . . . . . . . . . . . . . . . . . . . 11

4 Construction of a solver at the vertices $\quad 11$

4.1 Notations around a vertex . . . . . . . . . . . . . . . . . . . . . . 11

4.2 Conservation relations . . . . . . . . . . . . . . . . . . . . . . . 12

4.3 Entropy inequality. . . . . . . . . . . . . . . . . . . . . . . . . 13

4.4 Evaluation of the velocity and pressures at the vertices. . . . . . . . . . . . . . . . . 14

4.5 Interpretation of the solver in two simple cases . . . . . . . . . . . . . . . . 16

4.5.1 One-dimensional flow with planar symmetry . . . . . . . . . . . . . . . 16

4.5.2 One-dimensional flow with cylindrical symmetry . . . . . . . . . . . . . . 17 
4.6 Boundary conditions . . . . . . . . . . . . . . . . . . . . . . . 18



4.6 .2 Case of a prescribed normal velocity . . . . . . . . . . . . . . . . . . . . . . . . . . . . . . . . . . . . . .

4.7 Some remarks . . . . . . . . . . . . . . . . . . . . . . 20

5 Time discretization $\quad \mathbf{2 2}$

5.1 Mesh motion and variation of volume . . . . . . . . . . . . . . . . . . . . . . 22

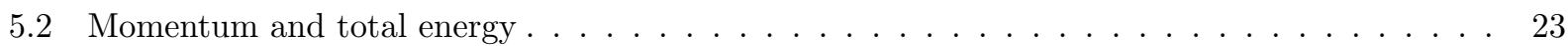

5.3 Time step limitation . . . . . . . . . . . . . . . . . . . . . . . . 23

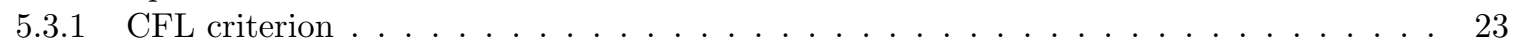

5.3.2 Criterion on the variation of volume . . . . . . . . . . . . . . . . . . . 24

5.4 Description of the algorithm . . . . . . . . . . . . . . . . . . . . . 24

6 Numerical results $\quad \mathbf{2 5}$

6.1 Multi-material Sod's shock tube problem . . . . . . . . . . . . . . . . . . 25

6.2 Noh's test case . . . . . . . . . . . . . . . . . . . . . . . . . . . 28

6.3 Kidder's isentropic compression . . . . . . . . . . . . . . . . . . . . . . . . . . . . . . . . . . . . . . . . .



6.5 Sedov's test case . . . . . . . . . . . . . . . . . . . . . . . . 36

6.6 Perturbation of the isentropic Kidder's compression . . . . . . . . . . . . . . . . . . . 39

6.6.1 Mode $n=4, \xi_{1}=0, \xi_{2}=1, a_{0}=10^{-6} \ldots \ldots \ldots \ldots \ldots$. . . . . . . . . . . . . . . . . . . . . . .

6.6.2 Mode $n=8, \xi_{1}=0, \xi_{2}=1, a_{0}=10^{-6} \ldots \ldots \ldots \ldots \ldots$. . . . . . . . . . . . . . . . . . . . . . . . .

6.6.3 Mode $n=8, \xi_{1}=2.565293, \xi_{2}=1, a_{0}=10^{-6} \ldots \ldots \ldots \ldots$. . . . . . . . . 44

6.7 ICF-like test case . . . . . . . . . . . . . . . . . . . . . . . 45

\section{Conclusion and perspectives}

\section{Introduction}

The physical model that is considered throughout this paper is based on the equations of fluid dynamics written in Lagrangian form. This form is well adapted to the simulation of multi-material compressible fluid flows, such as those encountered in the domain of Inertial Confinement Fusion (ICF), see [19] and [25]. Our aim is to propose a new Lagrangian cell-centered scheme for two-dimensional gas dynamics equations. Before describing our method, let us briefly give an historical overview on the Lagrangian schemes.

Lagrangian schemes are characterized by a mesh that follows the fluid flow. By this mean, these methods deal with interfaces in a natural manner. The main numerical difficulty lies in the node motion discretization, especially for multidimensional situations. The most natural way to overcome this difficulty is to use a staggered discretization, where the momentum is defined at the nodes and the other variables (density, pressure and specific internal energy) are cell-centered. This type of scheme was first introduced by Von Neumann and Richtmyer in [27] for one-dimensional flows. The bidimensional extension was proposed by Wilkins in [29]. It is based on an internal energy formulation. The entropy production inherent to shock waves is ensured by an artificial viscosity. In its initial version, this scheme was not conservative and it admitted numerical spurious modes. However, in spite of these drawbacks, this scheme has been widely used in the domain of multi-material flow simulations during last forty years. Moreover, since a decade, many improvements have been done in order to solve the previous problems. In their paper [7], Caramana and Shashkov show that with an appropriate discretization of the subzonal forces resulting from subzonal pressures, hourglass motion and spurious vorticity can be eliminated. By using the method of support operators proposed in [6], they constructed a staggered scheme which ensures the conservation of total energy. Moreover, the discretization of artificial viscosity has been considerably improved. First, by introducing formulations for multidimensional shock wave computations in [5] and then by using a discretization based on a mimetic finite difference scheme in [4]. With all these improvements, the staggered Lagrangian scheme 
is an accurate and robust method, which can produce impressive results, even on unstructured polygonal grids, see for instance [21].

An alternative to the staggered discretization is to use a conservative cell-centered discretization. This method for Lagrangian gas dynamics in one dimension, has been introduced by Godunov, see [12] and [24]. The multidimensional extension of this method has been performed during the eighties, [2] and [11]. This multidimensional scheme is a cell-centered finite volume scheme on moving structured or unstructured meshes. It is constructed by integrating directly the system of conservation laws on each moving cell. The primary variables, density, momentum and total energy are defined in the cells. The flux across the boundary of the cell is computed by solving exactly or approximately a one-dimensional Riemann problem in the direction normal to the boundary. The main problem with this type of method lies in the fact that the node velocity needed to move the mesh cannot be directly calculated. In [2], the node velocity is computed via a special least squares procedure. It consists in minimizing the error between the normal velocity coming from the Riemann solver and the normal projection of the vertex velocity. It turns out that it leads to an artificial grid motion, which requires a very expensive treatment [10]. Moreover, with this approach the flux calculation is not consistent with the node motion.

Recently, new cell-centered methods have been proposed in [15] and [1]. These new approaches use a fully Lagrangian form of the gas dynamics equations, that is, the gradient and divergence operators are expressed in the Lagrangian coordinates. This type of discretization needs to compute the Jacobian matrix associated to the map between Lagrangian and Eulerian spaces. However, these methods are purely Lagrangian and cannot be interpreted as moving mesh methods. This drawback has motivated an other approach proposed by Després and Mazeran. In [9], they made a theoretical analysis of the Lagrangian gas dynamics equations written in a fully Lagrangian form and they derived a new conservative and entropy consistent two-dimensional Lagrangian scheme of the finite volume type. It is a moving grid scheme based on a nodal solver. The node velocity is computed in a coherent manner with the face fluxes. The numerical results shown in [9] are quite impressive, in particular, those related to the difficult Saltzmann's test case. However, it appears that in the case of one-dimensional flows, this scheme leads a to nodal velocity, which depends on the cell aspect ratio. This drawback has motivated our study to develop a new cell-centered scheme that retains the good feature of Després-Mazeran scheme but resolves the aspect ratio problem. Here, we propose a new Lagrangian cell-centered scheme in which the vertex velocities and the numerical fluxes through the cell interfaces are not computed independently as usual but in a consistent manner with an original solver located at the nodes. The main new feature of the algorithm is the introduction of four pressures on each edge, two for each node on each side of the edge. This is the main difference from [9]. We show, in the limit of a one-dimensional flow computed by our two-dimensional solver, or for flows in a cylindrical geometry, that the scheme recovers the classical Godunov approximate Riemann solver. Moreover, our scheme locally conserves momentum, total energy and it satisfies a local entropy inequality. The boundary conditions are taken into account in a natural way. This scheme is first order in time and space.

The paper is organized as follows. First we recall the gas dynamics equations written in the Lagrangian form. In the second section we derive space approximations based on face and node fluxes. Then we build a nodal solver using conservation arguments and a discrete entropy inequality. In the fourth section we give the time discretization. Last, we validate our new scheme with several test cases. They are representative test cases for compressible fluid flows and demonstrate the robustness and the accuracy of this new solver.

\section{Derivation of the Euler equations in the Lagrangian formalism}

The aim of this section is to recall briefly how the gas dynamics equations are obtained in the Lagrangian formalism. For such a derivation, we follow the approach developed in [8].

Let $\mathcal{D}$ be a region of the two-dimensional space $\mathbb{R}^{2}$, filled with an inviscid fluid and equipped with an orthonormal frame $(O, X, Y)$. The Lagrangian formalism consists in following the time evolution of fluid particles along their trajectories. Consider a fluid particle which is moving through the point $M$ at time $t>0$ and whose initial position is point $m$. The coordinates of point $M$ are denoted by $(X, Y)$, they are 
named Eulerian coordinates. The coordinates of point $m$ are denoted by $(x, y)$, they are named Lagrangian coordinates. The Eulerian coordinates are obtained from the trajectory equations

$$
\begin{aligned}
& \frac{d X}{d t}=u(X, Y, t), \quad X(x, y, 0)=x, \\
& \frac{d Y}{d t}=v(X, Y, t), \quad Y(x, y, 0)=y,
\end{aligned}
$$

where $(u, v)$ are the coordinates of the fluid velocity $\boldsymbol{V}$. If the velocity field is smooth enough, the system (1) admits a unique solution $(X(x, y, t), Y(x, y, t))$. This enables to define the map

$$
\mathcal{M}_{t}:(x, y) \mapsto(X, Y),
$$

where $(X, Y)$ is the unique solution of (1). With fixed $t$, this map advances each fluid particle from its position at time $t=0$ to its position at time $t$. If $\omega$ denotes a region in $\mathcal{D}$, then $\mathcal{M}_{t}(\omega)=\Omega$ is the volume $\omega$ moving with the fluid. We assume that for each $t>0, \mathcal{M}_{t}$ is invertible. Let us introduce the Jacobian of this map

$$
J(x, y, t)=\left|\begin{array}{ll}
\frac{\partial X}{\partial x} & \frac{\partial X}{\partial y} \\
\frac{\partial Y}{\partial x} & \frac{\partial Y}{\partial y}
\end{array}\right| .
$$

We notice that $J(x, y, 0)=1$ and since $\mathcal{M}_{t}$ is invertible we have for each $t>0, J(x, y, t)>0$. Time differentiation of (3) gives the classical result

$$
\frac{d J}{d t}-J \nabla \cdot \boldsymbol{V}=0
$$

where $\nabla \cdot$ denotes the divergence operator, namely $\nabla \cdot \boldsymbol{V}=\frac{\partial u}{\partial X}+\frac{\partial v}{\partial Y}$.

Let us consider a flow variable $\varphi$ depending on Eulerian coordinates: $\varphi \equiv \varphi(X, Y, t)$. Time differentiation of $\varphi$ gives

using (1) one obtains

$$
\frac{d \varphi}{d t}=\frac{\partial \varphi}{\partial t}+\frac{d X}{d t} \frac{\partial \varphi}{\partial X}+\frac{d Y}{d t} \frac{\partial \varphi}{\partial Y}
$$

$$
\frac{d \varphi}{d t}=\frac{\partial \varphi}{\partial t}+\nabla \varphi \cdot \boldsymbol{V}
$$

where $\boldsymbol{\nabla}$ is the gradient operator, namely $\boldsymbol{\nabla} \varphi=\left(\frac{\partial \varphi}{\partial X}, \frac{\partial \varphi}{\partial Y}\right)^{t}$. The time derivative of $\varphi$ depending on Eulerian coordinates is named the material derivative, it represents the variation of $\varphi$ along a fluid trajectory. Finally, combining (4) and (5) it is straightforward to write

$$
\frac{d}{d t}(\varphi J)=J\left[\frac{\partial \varphi}{\partial t}+\nabla \cdot(\varphi \boldsymbol{V})\right] .
$$

With this last equations we are able to transform gas dynamics equations written in Eulerian formalism. These equations for an inviscid compressible fluid (see [18]) are

$$
\begin{aligned}
& \frac{\partial \rho}{\partial t}+\nabla \cdot(\rho \boldsymbol{V})=0, \\
& \frac{\partial \rho}{\partial t}(\rho \boldsymbol{V})+\nabla \cdot(\rho \boldsymbol{V} \otimes \boldsymbol{V})+\nabla P=\mathbf{0}, \\
& \frac{\partial \rho}{\partial t}(\rho E)+\nabla \cdot(\rho E \boldsymbol{V})+\nabla \cdot(P \boldsymbol{V})=0 .
\end{aligned}
$$


In (7), $\rho$ denotes the density, $P$ is the pressure and $E$ is the specific total energy. We denote by $\varepsilon=E-\frac{1}{2}\|\boldsymbol{V}\|^{2}$ the specific internal energy. The thermodynamic closure of $(7)$ is given by the equation of state, $P \equiv P(\rho, \varepsilon)$.

Now, using (6) for the conservative variables $\varphi=1, \rho, \rho \boldsymbol{V}, \rho E$ and after substitution in (7), one obtains the gas dynamics system in Lagrangian formalism:

$$
\begin{aligned}
& \frac{d J}{d t}-J \nabla \cdot \boldsymbol{V}=0, \\
& \frac{d}{d t}(\rho J)=0, \\
& \frac{d}{d t}(\rho J \boldsymbol{V})+J \nabla P=\mathbf{0}, \\
& \frac{d}{d t}(\rho J E)+J \nabla \cdot(P \boldsymbol{V})=0 .
\end{aligned}
$$

We notice that these equations are only in a semi-Lagrangian formalism since the gradient and divergence operates on variables which depends on Eulerian coordinates. In order to write these equations in a full Lagrangian way, one has to express the gradient and divergence operators with Lagrangian coordinates using the map $\mathcal{M}_{t}$. For such an approach, the reader should referred to [15], [20] and [9].

In order to perform the space discretization in the next section, we give the integral form of system (8). This form is obtained by integration of (8) on the Lagrangian domain $\omega$. Knowing that $\mathcal{M}_{t}(\omega)=\Omega$ and $J \mathrm{~d} \omega=\mathrm{d} \Omega$, using Green formula one gets

$$
\begin{aligned}
& \frac{d}{d t} \int_{\Omega} \mathrm{d} \Omega-\int_{\partial \Omega} \boldsymbol{V} \cdot \boldsymbol{N} \mathrm{d} l=0 \\
& \frac{d}{d t} \int_{\Omega} \rho \mathrm{d} \Omega=0 \\
& \frac{d}{d t} \int_{\Omega(t)} \rho \boldsymbol{V} \mathrm{d} \Omega+\int_{\partial \Omega} P \boldsymbol{N} \mathrm{d} l=\mathbf{0} \\
& \frac{d}{d t} \int_{\Omega(t)} \rho E \mathrm{~d} \Omega+\int_{\partial \Omega} P \boldsymbol{V} \cdot \boldsymbol{N} \mathrm{d} l=0,
\end{aligned}
$$

where $\partial \Omega$ is the boundary of $\Omega, \boldsymbol{N}$ is the unit outward normal to $\partial \Omega$ and $\mathrm{d} l$ is the length element on $\partial \Omega$. The equations (9) are well known and have been used in this form in many papers, see for example [14]. Let $m_{\Omega}$ denotes the mass of fluid enclosed in $\Omega$, equation (9)-(ii) simply states mass conservation, hence for each time one gets $m_{\Omega}=m_{\omega}$. Let us introduce the area of domain $\Omega$ :

$$
\mathcal{A}_{\Omega}=\int_{\Omega} \mathrm{d} \Omega
$$

We define the density $\rho_{\Omega}=\frac{m_{\omega}}{\mathcal{A}_{\Omega}}$ and the specific volume $\tau_{\Omega}=\frac{1}{\rho_{\Omega}}$. We also define the mass averaged velocity and total energy

$$
\begin{aligned}
& \boldsymbol{V}_{\boldsymbol{\Omega}}=\frac{1}{m_{\omega}} \int_{\Omega} \rho \boldsymbol{V} \mathrm{d} \Omega, \\
& E_{\Omega}=\frac{1}{m_{\omega}} \int_{\Omega} \rho E \mathrm{~d} \Omega .
\end{aligned}
$$

With these notations and mass conservation, one gets the final integral form which will be useful for space 
discretization:

$$
\begin{aligned}
& m_{\omega} \frac{d}{d t} \tau_{\Omega}-\int_{\partial \Omega} \boldsymbol{V} \cdot \boldsymbol{N} \mathrm{d} l=0, \\
& m_{\omega} \frac{d}{d t} \boldsymbol{V}_{\boldsymbol{\Omega}}+\int_{\partial \Omega} P \boldsymbol{N} \mathrm{d} l=\mathbf{0}, \\
& m_{\omega} \frac{d}{d t} E_{\Omega}+\int_{\partial \Omega} P \boldsymbol{V} \cdot \boldsymbol{N} \mathrm{d} l=0,
\end{aligned}
$$

where $m_{\omega}$ is the mass of fluid enclosed initially in $\omega$.

\section{Spatial approximation}

Using classical finite volume approach, we derive in this section the spatial approximation of the gas dynamics equations written in Lagrangian formalism. Let us introduce some notations.

\subsection{Notations, assumptions and problem statement}

Let $\left\{\omega_{i}, i=1 \ldots I\right\}$ be a collection of non overlapping polygons whose reunion covers $\omega$, the sub-domain of $\mathbb{R}^{2}$ that is initially filled by the fluid. We set $\Omega_{i}=\mathcal{M}_{t}\left(\omega_{i}\right)$, where $\mathcal{M}_{t}$ is the map defined by $(2)$. $\Omega_{i}$ is also a polygonal cell ${ }^{1}$ whose vertices are denoted by $M_{r}, r=1 \ldots R(i)$. We denote by $R(i)$ the number of vertices (or faces) of the cell $\Omega_{i}$. The vertices are indexed counter clockwise, see Figure 1 . The numbering is also periodic, i.e. $M_{R(i)+1}=M_{1}$ and $M_{0}=M_{R(i)}$. The frame $(O, X, Y)$ is equipped with an orthonormal basis $\left(\boldsymbol{e}_{\boldsymbol{X}}, \boldsymbol{e}_{\boldsymbol{Y}}\right)$ which is completed by the vector $\boldsymbol{e}_{\boldsymbol{Z}}=\boldsymbol{e}_{\boldsymbol{X}} \times \boldsymbol{e}_{\boldsymbol{Y}}$. We denote the Eulerian coordinates of the vertex $M_{r}$ by $\left(X_{r}, Y_{r}\right)$. Note that $M_{r}$ and its coordinates are time dependent. Considering any edge $\left[M_{r}, M_{r+1}\right]$ of $\Omega_{i}$, we denote its length by $L_{r, r+1}, \boldsymbol{T}_{\boldsymbol{r}, \boldsymbol{r}+\mathbf{1}}$ represents its unit tangent vector in a counter clockwise orientation, and $\boldsymbol{N}_{\boldsymbol{r}, \boldsymbol{r}+\mathbf{1}}$ is its unit outward normal vector. Following these definitions and conventions, we have

$$
\begin{aligned}
& L_{r, r+1} \boldsymbol{T}_{\boldsymbol{r}, \boldsymbol{r}+\mathbf{1}}=\boldsymbol{M}_{\boldsymbol{r}} \boldsymbol{M}_{\boldsymbol{r}+\mathbf{1}}, \\
& L_{r, r+1} \boldsymbol{N}_{\boldsymbol{r}, \boldsymbol{r + 1}}=L_{r, r+1} \boldsymbol{T}_{\boldsymbol{r}, \boldsymbol{r}+\mathbf{1}} \times \boldsymbol{e}_{\boldsymbol{Z}} .
\end{aligned}
$$

The fluid in cell $\Omega_{i}$ is described by the discrete variables $\left(\tau_{i}, \boldsymbol{V}_{\boldsymbol{i}}, E_{i}\right)$, respectively the averaged specific volume, velocity and specific total energy. A set of discrete equations is written for these discrete unknowns, using the integral formulation (10) applied for cell $\Omega_{i}$ :

$$
\begin{aligned}
& m_{i} \frac{d}{d t} \tau_{i}-\int_{\partial \Omega_{i}} \boldsymbol{V} \cdot \boldsymbol{N} \mathrm{d} l=0, \\
& m_{i} \frac{d}{d t} \boldsymbol{V}_{\boldsymbol{i}}+\int_{\partial \Omega_{i}} P \boldsymbol{N} \mathrm{d} l=\mathbf{0}, \\
& m_{i} \frac{d}{d t} E_{i}+\int_{\partial \Omega_{i}} P \boldsymbol{V} \cdot \boldsymbol{N} \mathrm{d} l=0,
\end{aligned}
$$

where $m_{i}$ denotes the mass of the cell $\Omega_{i}$. We also introduce the density $\rho_{i}=\frac{1}{\tau_{i}}$, the specific internal energy $\varepsilon_{i}=E_{i}-\frac{1}{2}\left\|\boldsymbol{V}_{\boldsymbol{i}}\right\|^{2}$ and the pressure given by the equation of state $P_{i}=P\left(\rho_{i}, \varepsilon_{i}\right)$. Since all fluid variables are assumed to be constant in cell $\Omega_{i}$ we obtain a spatial approximation which is first order accurate.

\footnotetext{
${ }^{1}$ Here, we assume that $\Omega_{i}$ is still a polygon. This means that we implicitly assume that the velocity field variation is linear in space. This is not restrictive here, because the aim of the paper is to develop a scheme that is first order in space.
} 


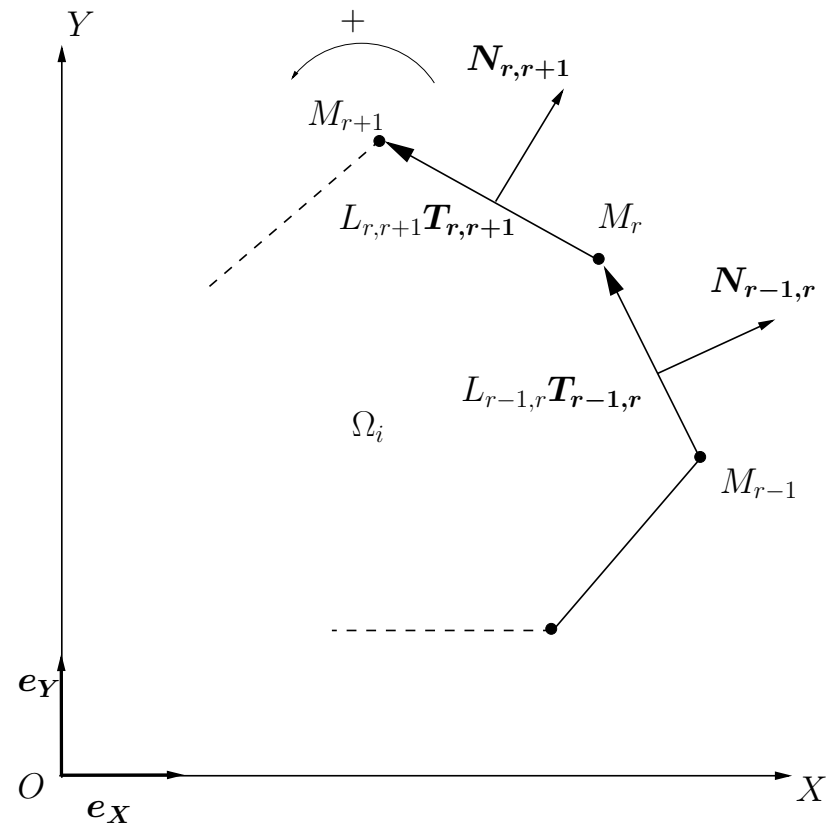

Figure 1: Notations

Finally, we introduce some notations to express the discrete face fluxes. Let us denote by $\boldsymbol{V}_{r, r+1}^{\star} \cdot \boldsymbol{N}_{r, r+1}$, $P_{r, r+1}^{\star} \boldsymbol{N}_{r, r+1}$ and $(P \boldsymbol{V})_{r, r+1}^{\star} \cdot \boldsymbol{N}_{r, r+1}$ the volume, momentum and total energy fluxes on the face $\left[M_{r}, M_{r+1}\right]$. They are defined by the following equations:

$$
\begin{aligned}
& L_{r, r+1} \boldsymbol{V}_{r, \boldsymbol{r}+\mathbf{1}}^{\star} \cdot \boldsymbol{N}_{\boldsymbol{r}, \boldsymbol{r + 1}}=\int_{M_{r}}^{M_{r+1}} \boldsymbol{V} \cdot \boldsymbol{N} \mathrm{d} l, \\
& L_{r, r+1} \boldsymbol{P}_{r, r+1}^{\star} \boldsymbol{N}_{r, \boldsymbol{r}+\mathbf{1}}=\int_{M_{r}}^{M_{r+1}} P \boldsymbol{N} \mathrm{d} l, \\
& L_{r, r+1}(P \boldsymbol{V})_{r, r+1}^{\star} \cdot \boldsymbol{N}_{r, \boldsymbol{r}+\mathbf{1}}=\int_{M_{r}}^{M_{r+1}} P \boldsymbol{V} \cdot \boldsymbol{N} \mathrm{d} l .
\end{aligned}
$$

With these notations the previous system is now written in the following manner:

$$
\begin{aligned}
& m_{i} \frac{d}{d t} \tau_{i}-\sum_{r=1}^{R(i)} L_{r, r+1} \boldsymbol{V}_{r, \boldsymbol{r}+\mathbf{1}}^{\star} \cdot \boldsymbol{N}_{\boldsymbol{r}, \boldsymbol{r}+\mathbf{1}}=0, \\
& m_{i} \frac{d}{d t} \boldsymbol{V}_{\boldsymbol{i}}+\sum_{r=1}^{R(i)} L_{r, r+1} P_{r, r+1}^{\star} \boldsymbol{N}_{\boldsymbol{r}, \boldsymbol{r}+\mathbf{1}}=\mathbf{0}, \\
& m_{i} \frac{d}{d t} E_{i}+\sum_{r=1}^{R(i)} L_{r, r+1}(P \boldsymbol{V})_{r, r+1}^{\star} \cdot \boldsymbol{N}_{\boldsymbol{r}, \boldsymbol{r}+\mathbf{1}}=0 .
\end{aligned}
$$

We notice that with this Lagrangian discretization we have to take account of the cell motion. For this 
purpose we write the node motion equation from:

$$
\begin{aligned}
& \frac{d}{d t} X_{r}=u_{r}^{\star}, \quad X_{r}(0)=x_{r}, \\
& \frac{d}{d t} Y_{r}=v_{r}^{\star}, \quad Y_{r}(0)=y_{r},
\end{aligned}
$$

where $\left(u_{r}^{\star}, v_{r}^{\star}\right)$ are the components of the velocity $\boldsymbol{V}_{r}^{\star}$ of the vertex $M_{r}$.

In order to complete the space approximation, the following important problems arise:

1. How to compute the face fluxes defined by (12)?

2. How to compute the node velocities $\boldsymbol{V}_{\boldsymbol{r}}^{\star}$ ?

3. These velocities being known, how can we ensure the compatibility between the mesh motion and the volume variation of the cells?

Thus, our task consists in building a numerical solver that can compute the fluxes $\boldsymbol{V}_{r, \boldsymbol{r}+\mathbf{1}}^{\star}, P_{r, r+1}^{\star}$ and $(P \boldsymbol{V})_{r, r+1}^{\star}$ but also the node velocities $\boldsymbol{V}_{\boldsymbol{r}}^{\star}$. Moreover, this must be done coherently. We resolve these questions in the next sections.

\subsection{Approximation of the volume equation}

Using a geometrical interpretation of (13)-(ii), we can link the volume flux $\boldsymbol{V}_{\boldsymbol{r}, \boldsymbol{r}+\mathbf{1}}^{\star} \cdot \boldsymbol{N}_{\boldsymbol{r}, \boldsymbol{r}+\mathbf{1}}$ to the node velocity $\boldsymbol{V}_{\boldsymbol{r}}^{\star}$.

First, we notice that $m_{i} \tau_{i}=\mathcal{A}_{i}$, where $\mathcal{A}_{i}$ is the area of the cell $\Omega_{i}$. Thus, (13)-(ii) provides the time variation of $\mathcal{A}_{i}$

$$
\frac{d}{d t} \mathcal{A}_{i}=\sum_{r=1}^{R(i)} L_{r, r+1} \boldsymbol{V}_{\boldsymbol{r}, \boldsymbol{r}+\mathbf{1}}^{\star} \cdot \boldsymbol{N}_{\boldsymbol{r}, \boldsymbol{r}+\mathbf{1}} .
$$

The area of the cell $\Omega_{i}$ can also be evaluated from the location of the vertices $M_{r}$ using the relation (see [28])

$$
\mathcal{A}_{i}=\frac{1}{2} \sum_{r=1}^{R} \boldsymbol{O} \boldsymbol{M}_{\boldsymbol{r}} \times \boldsymbol{O} \boldsymbol{M}_{\boldsymbol{r + 1}} \cdot \boldsymbol{e}_{\boldsymbol{Z}}
$$

This amounts to sum the area of the triangles $\left(O, M_{r}, M_{r+1}\right)$, see Figure 1, over all the vertices of the cell. Using the coordinates of the vertices and after time differentiation, we get

$$
\frac{d}{d t} \mathcal{A}_{i}=\frac{1}{2} \sum_{r=1}^{R(i)}\left(Y_{r+1} \frac{d}{d t} X_{r}-X_{r+1} \frac{d}{d t} Y_{r}+X_{r} \frac{d}{d t} Y_{r+1}-Y_{r} \frac{d}{d t} X_{r+1}\right) .
$$

We rearrange the last two terms of the sum using the transformation $r \rightarrow r-1$ and the periodicity of the indices, and we get

$$
\frac{d}{d t} \mathcal{A}_{i}=\frac{1}{2} \sum_{r=1}^{R(i)}\left[\left(Y_{r+1}-Y_{r}+Y_{r}-Y_{r-1}\right) \frac{d}{d t} X_{r}-\left(X_{r+1}-X_{r}+X_{r}-X_{r-1}\right) \frac{d}{d t} Y_{r}\right] .
$$

This formula can be rewritten as

$$
\frac{d}{d t} \mathcal{A}_{i}=\frac{1}{2} \sum_{r=1}^{R(i)} \boldsymbol{V}_{\boldsymbol{r}}^{\star} \cdot\left(L_{r-1, r} \boldsymbol{N}_{\boldsymbol{r}-\mathbf{1}, \boldsymbol{r}}+L_{r, r+1} \boldsymbol{N}_{r, \boldsymbol{r}+\mathbf{1}}\right),
$$


and using the change of indices $r-1 \rightarrow r$ for the first term of the sum, we have the equivalent formulation

$$
\frac{d}{d t} \mathcal{A}_{i}=\sum_{r=1}^{R(i)} \frac{1}{2} L_{r, r+1}\left(\boldsymbol{V}_{\boldsymbol{r}}^{\star}+\boldsymbol{V}_{\boldsymbol{r}+1}^{\star}\right) \cdot \boldsymbol{N}_{r, \boldsymbol{r}+\mathbf{1}} .
$$

The comparison between (15) and (17) enables to give the face velocities in term of the vertex velocities, namely

$$
V_{r, r+1}^{\star}=\frac{1}{2}\left(V_{r}^{\star}+V_{r+1}^{\star}\right) .
$$

This fundamental relation enables to write two equivalent discretizations of the specific volume variation. It can be given in term of the flux through the faces, or equivalently, in term of vertex fluxes. Moreover, the two discretizations are compatible with the node motion. We get one of the results of [9], but with a much simpler geometrical argument. We note that (18) is consistent with the assumption that the velocity field is linear along face $\left[M_{r}, M_{r+1}\right]$.

Hence, one can consider two methods for computing the face velocities:

- The first one relies on the evaluation of the normal velocity $\boldsymbol{V}_{r, r+1}^{\star} \cdot \boldsymbol{N}_{r, \boldsymbol{r}+\mathbf{1}}$ Using a one-dimensional Riemann solver at faces. The vertex velocities $\boldsymbol{V}_{\boldsymbol{r}}^{\star}$ have to be computed by solving a linear system built from equations (18) written for all the faces. This system is generally over-determined. Consequently, a least squares technique is used, see e.g. [2]. Then, the variation of cell areas must be recomputed to keep the coherence with vertex motion.

- In the second method, the velocities $\boldsymbol{V}_{r}^{\star}$ are first evaluated using some still-to-define solver. The face velocities are then computed from (18). This is the technique we will use in the sequel of the paper because this method will guarantee the compatibility between vertex motion and cell areas variation.

\subsection{Approximation of momentum flux}

Following the same methodology, we approximate the momentum flux defined at the vertices in such a way that it is compatible with the approximation at faces. Thus, similarly to (18) we set

$$
P_{r, r+1}^{\star, i}=\frac{1}{2}\left(P_{r, r+\frac{1}{2}}^{\star, i}+P_{r+\frac{1}{2}, r+1}^{\star, i}\right)
$$

where $P_{r, r+\frac{1}{2}}^{\star, i}\left(\right.$ resp. $\left.P_{r+\frac{1}{2}, r+1}^{\star, i}\right)$ represents the pressure on the half face $\left[M_{r}, M_{r+\frac{1}{2}}\right]\left(\operatorname{resp} .\left[M_{r+\frac{1}{2}}, M_{r+1}\right]\right)$ seen from the cell $\Omega_{i}$, see Figure 2. We have added the superscript $i$ to remove any possible ambiguity. Hence, $\frac{1}{2} L_{r, r+1} P_{r, r+\frac{1}{2}}^{\star, i} N_{r, r+1}$ represents the momentum flux for half face $\left[M_{r}, M_{r+\frac{1}{2}}\right]$ seen from the cell $\Omega_{i}$. We observe that at node $M_{r}$, seen from the cell $\Omega_{i}$, there exists two pressures: $P_{r, r+\frac{1}{2}}^{\star, i}$ and $P_{r-\frac{1}{2}, r}^{\star, i}$. Because of this, our approach is different from that of [9], where only one pressure is defined at the vertex $M_{r}$.

Using this definition, the equation for momentum (13)-(iii) writes

$$
m_{i} \frac{d}{d t} \boldsymbol{V}_{\boldsymbol{i}}+\sum_{r=1}^{R(i)} \frac{1}{2} L_{r, r+1}\left(P_{r, r+\frac{1}{2}}^{\star, i}+P_{r+\frac{1}{2}, r+1}^{\star, i}\right) \boldsymbol{N}_{\boldsymbol{r}, \boldsymbol{r}+\mathbf{1}}=\mathbf{0} .
$$

If we shift the index $r$ (i.e. $r \rightarrow r-1$ ) in the second term of the summation, we get

$$
m_{i} \frac{d}{d t} \boldsymbol{V}_{\boldsymbol{i}}+\sum_{r=1}^{R(i)} \frac{1}{2}\left(L_{r-1, r} P_{r-\frac{1}{2}, r}^{\star, i} \boldsymbol{N}_{\boldsymbol{r - 1 , r}}+L_{r, r+1} P_{r, r+\frac{1}{2}}^{\star, i} \boldsymbol{N}_{r, \boldsymbol{r}+\mathbf{1}}\right)=\mathbf{0} .
$$

It amounts to sum the fluxes over all nodes of the cell. The equations (20) and (21) are equivalent discretizations. They use either face fluxes or vertex fluxes. The evaluation of these fluxes uses two pressures at a face or at a node for cell $\Omega_{i}$. 


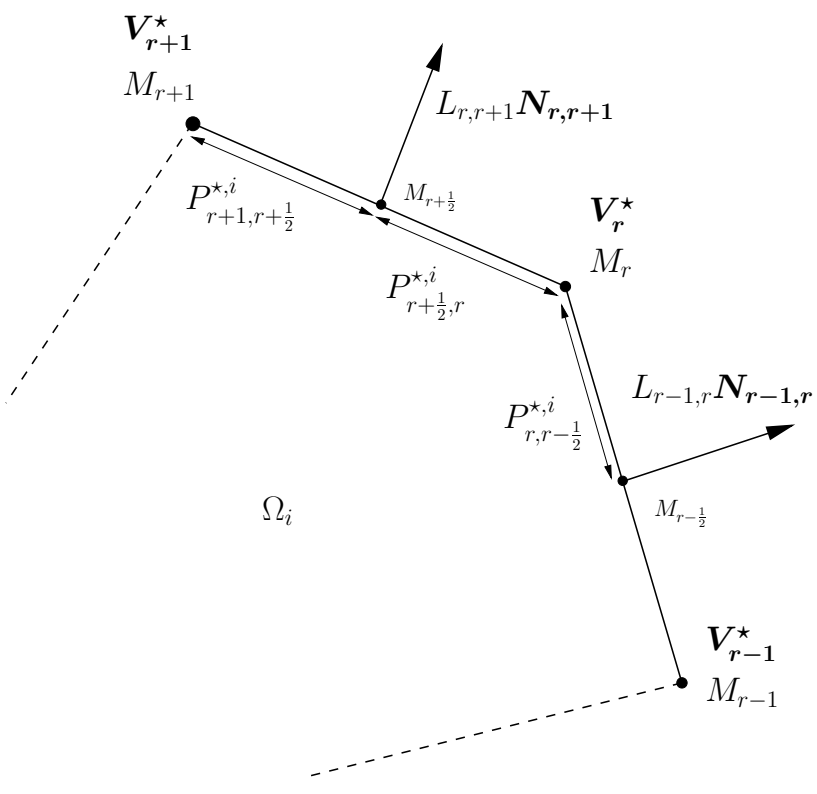

Figure 2: Pressures at faces

Remark 1. We notice that in the case of a one-dimensional Riemann solver at faces, as classically done, only one single pressure is computed for $\left[M_{r}, M_{r+1}\right]$. The conservation of the momentum is a simple consequence of this approximation. Here, this is not any longer the case. In fact, if the cell $\Omega_{j}$ shares face $\left[M_{r}, M_{r+1}\right]$ with $\Omega_{i}$, the pressures on this face seen from $\Omega_{i}$ may be in general different of those seen from $\Omega_{j}$. That is why we have put the superscript denoting the cell in the definition of edge pressures. This is an important fact, which is one of the key point of our construction: in adding degrees of freedom as here, we will see later in text that this enables us to build a node solver which provides us simultaneously the node velocity and the local conservation of momentum and energy.

\subsection{Approximation of the energy flux}

In order to get an equivalent discretization in term of face and nodal fluxes for the total energy equation, we set

$$
(P \boldsymbol{V})_{r, r+1}^{\star, i}=\frac{1}{2}\left(P_{r, r+\frac{1}{2}}^{\star, i} \boldsymbol{V}_{\boldsymbol{r}}^{\star}+P_{r+\frac{1}{2}, r+1}^{\star, i} \boldsymbol{V}_{r+1}^{\star}\right) .
$$

Using (22), the total energy equation in its face flux formulation writes

$$
m_{i} \frac{d}{d t} E_{i}+\sum_{r=1}^{R(i)} \frac{1}{2} L_{r, r+1}\left(P_{r, r+\frac{1}{2}}^{\star, i} \boldsymbol{V}_{\boldsymbol{r}}^{\star}+P_{r+\frac{1}{2}, r+1}^{\star, i} \boldsymbol{V}_{\boldsymbol{r}+\mathbf{1}}^{\star}\right) \cdot \boldsymbol{N}_{\boldsymbol{r}, \boldsymbol{r}+\mathbf{1}}=0 .
$$

Shifting once more the index $r$ in the second term of the sum, we get the node flux formulation

$$
m_{i} \frac{d}{d t} E_{i}+\sum_{r=1}^{R(i)} \frac{1}{2}\left(L_{r-1, r} P_{r-\frac{1}{2}, r}^{\star, i} \boldsymbol{N}_{\boldsymbol{r}-\mathbf{1}, \boldsymbol{r}}+L_{r, r+1} P_{r, r+\frac{1}{2}}^{\star, i} \boldsymbol{N}_{\boldsymbol{r}, \boldsymbol{r + 1}}\right) \cdot \boldsymbol{V}_{\boldsymbol{r}}^{\star}=0 .
$$




\subsection{Summary}

We obtain an approximation of the Euler equations written in Lagrangian coordinates which relies on approximated vertex fluxes:

$$
\begin{aligned}
& m_{i} \frac{d}{d t} \tau_{i}-\sum_{r=1}^{R(i)} \frac{1}{2} L_{r, r+1}\left(\boldsymbol{V}_{\boldsymbol{r}}^{\star}+\boldsymbol{V}_{\boldsymbol{r}+\mathbf{1}}^{\star}\right) \cdot \boldsymbol{N}_{\boldsymbol{r}, \boldsymbol{r + 1}}=0, \\
& m_{i} \frac{d}{d t} \boldsymbol{V}_{\boldsymbol{i}}+\sum_{r=1}^{R(i)} \frac{1}{2}\left(L_{r-1, r} P_{r-\frac{1}{2}, r}^{\star, i} \boldsymbol{N}_{\boldsymbol{r}-\mathbf{1}, \boldsymbol{r}}+L_{r, r+1} P_{r, r+\frac{1}{2}}^{\star, i} \boldsymbol{N}_{r, \boldsymbol{r}+\mathbf{1}}\right)=\mathbf{0}, \\
& m_{i} \frac{d}{d t} E_{i}+\sum_{r=1}^{R(i)} \frac{1}{2}\left(L_{r-1, r} P_{r-\frac{1}{2}, r}^{\star, i} \boldsymbol{N}_{\boldsymbol{r}-\mathbf{1 , r}}+L_{r, r+1} P_{r, r+\frac{1}{2}}^{\star, i} \boldsymbol{N}_{\boldsymbol{r}, \boldsymbol{r}+\mathbf{1}}\right) \cdot \boldsymbol{V}_{\boldsymbol{r}}^{\star}=0 .
\end{aligned}
$$

The system (25) is equivalent to (13) provided the face fluxes are defined by

$$
\begin{aligned}
& \boldsymbol{V}_{r, \boldsymbol{r}+\mathbf{1}}^{\star}=\frac{1}{2}\left(\boldsymbol{V}_{\boldsymbol{r}}^{\star}+\boldsymbol{V}_{\boldsymbol{r}+\mathbf{1}}^{\star}\right), \\
& P_{r, r+1}^{\star, i}=\frac{1}{2}\left(P_{r, r+\frac{1}{2}}^{\star, i}+P_{r+\frac{1}{2}, r+1}^{\star, i}\right), \\
& (P \boldsymbol{V})_{r, r+1}^{\star, i}=\frac{1}{2}\left(P_{r, r+\frac{1}{2}}^{\star, i} \boldsymbol{V}_{\boldsymbol{r}}^{\star}+P_{r+\frac{1}{2}, r+1}^{\star, i} \boldsymbol{V}_{\boldsymbol{r}+\mathbf{1}}^{\star}\right) .
\end{aligned}
$$

Moreover, this definition is coherent with the vertex motion.

Now, we are going to show how to compute, for each vertex $M_{r}$, the velocity $\boldsymbol{V}_{r}^{\star}$ and the pressures $P_{r-\frac{1}{2}, r}^{\star, i}$ et $P_{r, r+\frac{1}{2}}^{\star, i}$.

\section{Construction of a solver at the vertices}

The evaluation of $\boldsymbol{V}_{\boldsymbol{r}}^{\star}, P_{r-\frac{1}{2}, r}^{\star, i}$ and $P_{r, r+\frac{1}{2}}^{\star, i}$ relies on the following arguments:

- the global conservation of momentum,

- a local entropy inequality.

The first argument is a consequence of remark 1: the pressure on the face $\left[M_{r}, M_{r+1}\right]$ is not unique, contrary to the standard finite volume approach. Thus, we lose the automatic conservation of momentum and total energy. Of course, we must guarantee the conservation of momentum and total energy, so we have to add additional constraints on $\boldsymbol{V}_{\boldsymbol{r}}^{\star}, P_{r-\frac{1}{2}, r}^{\star, i}$ and $P_{r, r+\frac{1}{2}}^{\star, i}$.

The second argument is about the thermodynamical consistency of the scheme, which is necessary for a correct computation of the discontinuities. We will build a sufficient condition which will supply a local dissipation of entropy.

The two sets of constraints (conservation and dissipation of entropy) will lead, as we shall see in this section, to a linear system whose unique solution will give us $\boldsymbol{V}_{\boldsymbol{r}}^{\star}, P_{r-\frac{1}{2}, r}^{\star, i}$ and $P_{r, r+\frac{1}{2}}^{\star, i}$.

\subsection{Notations around a vertex}

In order to exhibit conservation equations for momentum and total energy around a node, let us introduce some new notations. We denote by $M_{q}$ a generic internal vertex of the mesh, for $q=1 \ldots Q$, where $Q$ is the total number of internal nodes. The case of the nodes located on the boundary $\partial \Omega$ will be treated in 


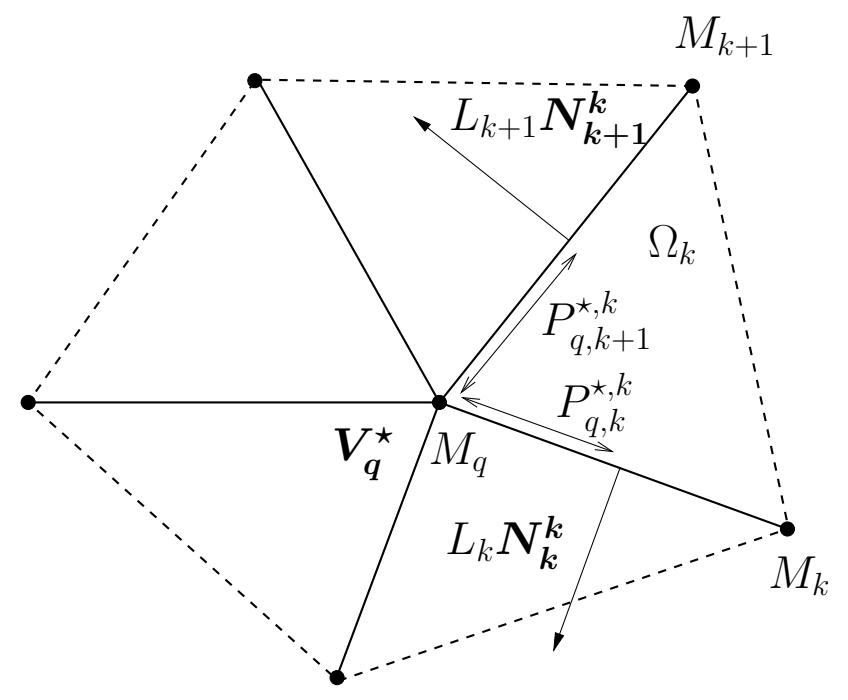

Figure 3: Notations around the vertex $M_{q}$

paragraph (4.6).There are $K(q)$ cells around $M_{q}$ which are denoted by $\Omega_{k}$, see Figure 3. For the cell $\Omega_{k}$ we denote by $\left[M_{q}, M_{k}\right]$ and $\left[M_{q}, M_{k+1}\right]$ the edges coming from node $M_{q}$. The outward normal to these edges are $\boldsymbol{N}_{\boldsymbol{k}}^{\boldsymbol{k}}, \boldsymbol{N}_{\boldsymbol{k}+\mathbf{1}}^{k}$ and their lengths $L_{k}, L_{k+1}$. Let $\boldsymbol{V}_{\boldsymbol{q}}^{\star}$ be the velocity of node $M_{q}$ and $P_{q, k}^{\star, k}\left(\right.$ resp. $\left.P_{q, k+1}^{\star, k}\right)$ the half pressures on edge $\left[M_{q}, M_{k}\right]$ (resp. $\left.\left[M_{q}, M_{k+1}\right]^{q}\right)$ seen from cell $\Omega_{k}$.

\subsection{Conservation relations}

Omitting the boundary conditions and summing equations (21) on each cells of the domain, we make a global balance of momentum

$$
\frac{d}{d t}\left(\sum_{i=1}^{I} m_{i} \boldsymbol{V}_{\boldsymbol{i}}\right)=-\sum_{i=1}^{I} \sum_{r=1}^{r(i)} \frac{1}{2}\left(L_{r-1, r}^{i} P_{r-\frac{1}{2}, r}^{\star, i} \boldsymbol{N}_{\boldsymbol{r}-\mathbf{1}, \boldsymbol{r}}^{\boldsymbol{i}}+L_{r, r+1}^{i} P_{r, r+\frac{1}{2}}^{\star, i} \boldsymbol{N}_{\boldsymbol{r}, \boldsymbol{r + 1}}^{i}\right) .
$$

We have added the superscript $i$ for the lengths and normals in order to remove any possible ambiguity. If we replace the global summation over cells by a global summation over nodes, using the notations previously defined, we can rewrite the right hand side in the following way

$$
\frac{d}{d t}\left(\sum_{i=1}^{I} m_{i} \boldsymbol{V}_{\boldsymbol{i}}\right)=-\sum_{q=1}^{Q} \sum_{k=1}^{K(q)} \frac{1}{2}\left(L_{k} P_{q, k}^{\star, k} \boldsymbol{N}_{k}^{k}+L_{k+1} P_{q, k+1}^{\star, k} \boldsymbol{N}_{\boldsymbol{k}+1}^{k}\right) .
$$

The (global) conservation of momentum is satisfied provided the right hand side vanishes. A sufficient condition is

$$
\sum_{k=1}^{K(q)} \frac{1}{2}\left(L_{k} P_{q, k}^{\star, k} \boldsymbol{N}_{\boldsymbol{k}}^{k}+L_{k+1} P_{q, k+1}^{\star, k} \boldsymbol{N}_{\boldsymbol{k}+1}^{k}\right)=\mathbf{0}
$$

This summation is done over all the cells $k$ surrounding vertex $M q$, see Figure 3 . The relation (27) can be interpreted as the local equilibrium of vertex $M_{q}$ under pressure forces. This is also a local conservation relation. 
It can be checked that condition (27) also implies the global conservation of total energy. To do so, we sum (24) on each cell, switch the summation for the cells and the vertices indices, and get:

$$
\frac{d}{d t}\left(\sum_{i=1}^{I} m_{i} E_{i}\right)=-\sum_{q=1}^{Q} \sum_{k=1}^{K(q)} \frac{1}{2}\left(L_{k} P_{q, k}^{\star, k} \boldsymbol{N}_{\boldsymbol{k}}^{\boldsymbol{k}}+L_{k+1} P_{q, k+1}^{\star, k} \boldsymbol{N}_{\boldsymbol{k}+\mathbf{1}}^{\boldsymbol{k}}\right) \cdot \boldsymbol{V}_{\boldsymbol{q}}^{\star}=0
$$

using (27) and because the velocity $\boldsymbol{V}_{q}^{\star}$ is single valued at $M_{q}$.

The conservation of volume is easily checked. To do so, we sum the first equation of (25) for each cell:

$$
\frac{d}{d t}\left(\sum_{i=1}^{I} m_{i} \tau_{i}\right)=\sum_{q=1}^{Q} \sum_{k=1}^{K(q)} \frac{1}{2}\left(L_{k} \boldsymbol{N}_{\boldsymbol{k}}^{\boldsymbol{k}}+L_{k+1} \boldsymbol{N}_{\boldsymbol{k}+\mathbf{1}}^{\boldsymbol{k}}\right) \cdot \boldsymbol{V}_{\boldsymbol{q}}^{\star} .
$$

We know that

$$
\sum_{k=1}^{K(q)} \frac{1}{2}\left(L_{k} \boldsymbol{N}_{\boldsymbol{k}}^{\boldsymbol{k}}+L_{k+1} \boldsymbol{N}_{\boldsymbol{k}+\mathbf{1}}^{\boldsymbol{k}}\right)=\mathbf{0}
$$

since the polygon whose vertices are $M_{k}, k=1 \ldots K(q)$ is closed (dotted line on Figure 3 ).

\subsection{Entropy inequality.}

We first compute the time variation of entropy in cell $\Omega_{i}$. Le us denote it by $\sigma_{i}$. If $T_{i}$ is the cell average temperature, we have

$$
m_{i} T_{i} \frac{d}{d t} \sigma_{i}=m_{i}\left(\frac{d}{d t} \varepsilon_{i}+P_{i} \frac{d}{d t} \tau_{i}\right)
$$

This quantity is evaluated in two steps. First, we compute the variation of internal energy $\frac{d}{d t} \varepsilon_{i}=\frac{d}{d t} E_{i}-$ $\boldsymbol{V}_{\boldsymbol{i}} \cdot \frac{d}{d t} \boldsymbol{V}_{\boldsymbol{i}}$, then we evaluate the pressure work $P_{i} \frac{d}{d t} \tau_{i}$

- Internal energy: we dot-multiply (21) by $\boldsymbol{V}_{\boldsymbol{i}}$ to get the kinetic energy variation:

$$
m_{i} \boldsymbol{V}_{\boldsymbol{i}} \cdot \frac{d}{d t} \boldsymbol{V}_{\boldsymbol{i}}=-\sum_{r=1}^{R(i)} \frac{1}{2}\left(L_{r-1, r} P_{r-\frac{1}{2}, r}^{\star, i} \boldsymbol{N}_{\boldsymbol{r}-\mathbf{1 , r}}+L_{r, r+1} P_{r, r+\frac{1}{2}}^{\star, i} \boldsymbol{N}_{\boldsymbol{r}, \boldsymbol{r + 1}}\right) \cdot \boldsymbol{V}_{\boldsymbol{i}}
$$

Then (30) is subtracted from the total energy variation equation and we have:

$$
m_{i} \frac{d}{d t} \varepsilon_{i}=-\sum_{r=1}^{R(i)} \frac{1}{2}\left(L_{r-1, r} P_{r-\frac{1}{2}, r}^{\star, i} \boldsymbol{N}_{\boldsymbol{r}-\mathbf{1}, \boldsymbol{r}}+L_{r, r+1} P_{r, r+\frac{1}{2}}^{\star, i} \boldsymbol{N}_{\boldsymbol{r}, \boldsymbol{r}+\mathbf{1}}\right) \cdot\left(\boldsymbol{V}_{\boldsymbol{r}}^{\star}-\boldsymbol{V}_{\boldsymbol{i}}\right) .
$$

- Pressure work: we multiply the first relation of (25) by $P_{i}$ :

$$
m_{i} P_{i} \frac{d}{d t} \tau_{i}=\sum_{r=1}^{R(i)} \frac{1}{2}\left(L_{r-1, r} P_{i} \boldsymbol{N}_{\boldsymbol{r}-\mathbf{1 , r}}+L_{r, r+1} P_{i} \boldsymbol{N}_{\boldsymbol{r}, \boldsymbol{r}+\mathbf{1}}\right) \cdot \boldsymbol{V}_{\boldsymbol{r}}^{\star}
$$

An equivalent formulation is

$$
m_{i} P_{i} \frac{d}{d t} \tau_{i}=\sum_{r=1}^{R(i)} \frac{1}{2}\left(L_{r-1, r} P_{i} \boldsymbol{N}_{\boldsymbol{r}-\mathbf{1}, \boldsymbol{r}}+L_{r, r+1} P_{i} \boldsymbol{N}_{\boldsymbol{r}, \boldsymbol{r}+\mathbf{1}}\right) \cdot\left(\boldsymbol{V}_{\boldsymbol{r}}^{\star}-\boldsymbol{V}_{\boldsymbol{i}}\right),
$$

knowing that:

$$
\sum_{r=1}^{R(i)} \frac{1}{2}\left(L_{r-1, r} \boldsymbol{N}_{\boldsymbol{r}-\mathbf{1}, \boldsymbol{r}}+L_{r, r+1} \boldsymbol{N}_{\boldsymbol{r}, \boldsymbol{r}+\mathbf{1}}\right)=\mathbf{0}
$$

because the boundary of $\Omega_{i}$ is a closed polygon. 
Last, the summation of (31) and (32) gives:

$$
m_{i} T_{i} \frac{d}{d t} \sigma_{i}=\sum_{r=1}^{R(i)} \frac{1}{2}\left[L_{r-1, r}\left(P_{i}-P_{r-\frac{1}{2}, r}^{\star, i}\right) \boldsymbol{N}_{r-1, r}+L_{r, r+1}\left(P_{j}-P_{r, r+\frac{1}{2}}^{\star, i}\right) \boldsymbol{N}_{r, \boldsymbol{r}+\mathbf{1}}\right] \cdot\left(\boldsymbol{V}_{\boldsymbol{r}}^{\star}-\boldsymbol{V}_{\boldsymbol{i}}\right) .
$$

A sufficient condition for the right hand side of this relation to be positive is:

$$
\left\{\begin{array}{l}
P_{i}-P_{r-\frac{1}{2}, r}^{\star, i}=\alpha_{i}\left(\boldsymbol{V}_{\boldsymbol{r}}^{\star}-\boldsymbol{V}_{\boldsymbol{i}}\right) \cdot \boldsymbol{N}_{\boldsymbol{r}-\mathbf{1}, \boldsymbol{r}}, \quad r=1 \ldots R(i), \\
P_{i}-P_{r, r+\frac{1}{2}}^{\star, i}=\alpha_{i}\left(\boldsymbol{V}_{\boldsymbol{r}}^{\star}-\boldsymbol{V}_{\boldsymbol{i}}\right) \cdot \boldsymbol{N}_{\boldsymbol{r}, \boldsymbol{r}+\mathbf{1}}, \quad r=1 \ldots R(i),
\end{array}\right.
$$

where $\alpha_{i}$ is a positive coefficient that has the dimension of a mass flux. These two relations can be interpreted as (discretized) Riemann invariants along the directions $\boldsymbol{N}_{\boldsymbol{r - 1 , r}}$ and $\boldsymbol{N}_{\boldsymbol{r}, \boldsymbol{r + 1}}$. By analogy with the acoustic solver [24], the parameter $\alpha_{i}$ is set to the acoustic impedance

$$
\alpha_{i}=\rho_{i} c_{i},
$$

where $c_{i}=\sqrt{\left(\frac{\partial P}{\partial \rho}\right)_{\sigma}}$ represents the isentropic sound speed. Under condition (33), the variation of entropy is:

$$
m_{i} T_{i} \frac{d}{d t} \sigma_{i}=\sum_{r=1}^{R(i)} \frac{1}{2} \rho_{i} c_{i}\left\{L_{r-1, r}\left[\left(\boldsymbol{V}_{\boldsymbol{r}}^{\star}-\boldsymbol{V}_{\boldsymbol{i}}\right) \cdot \boldsymbol{N}_{\boldsymbol{r}-\mathbf{1}, \boldsymbol{r}}\right]^{2}+L_{r, r+1}\left[\left(\boldsymbol{V}_{\boldsymbol{r}}^{\star}-\boldsymbol{V}_{\boldsymbol{i}}\right) \cdot \boldsymbol{N}_{\boldsymbol{r}, \boldsymbol{r + 1}}\right]^{2}\right\} .
$$

Remark 2. The relation (35) is very similar to the entropy production term resulting from a two-dimensional linear artificial viscosity term, see [5]. We notice that (35) implies a positive production of entropy, even in the case of isentropic flows. For such flows, our scheme, does not conserve entropy. This is typical for Godunov-type schemes.

If we rewrite the sufficient condition (33) using notations around a generic vertex $M_{q}$, we obtain

$$
\left\{\begin{array}{l}
P_{k}-P_{q, k}^{\star, k}=\alpha_{k}\left(\boldsymbol{V}_{\boldsymbol{q}}^{\star}-\boldsymbol{V}_{\boldsymbol{k}}\right) \cdot \boldsymbol{N}_{\boldsymbol{k}}^{\boldsymbol{k}}, \quad k=1 \ldots K(q), \\
P_{k}-P_{q, k+1}^{\star, k}=\alpha_{k}\left(\boldsymbol{V}_{\boldsymbol{q}}^{\star}-\boldsymbol{V}_{\boldsymbol{k}}\right) \cdot \boldsymbol{N}_{\boldsymbol{k}+\mathbf{1}}^{\boldsymbol{k}}, \quad k=1 \ldots \ldots K(q),
\end{array}\right.
$$

where $\alpha_{k}, P_{k}$ and $\boldsymbol{V}_{\boldsymbol{k}}$ stand for acoustic impedance, pressure and velocity in cell $\Omega_{k}$. We point out that for a vertex $M_{q}$ (not on the boundary of the computational domain), we have to compute $2 K(q)$ pressures, that is, together with the velocity $\boldsymbol{V}_{\boldsymbol{q}}^{\star}$, to evaluate $2 K(q)+2$ scalar unknowns. Equation (27) provides 2 scalar relations, and (36) gives $2 K(q)$ more. Hence, we can compute the pressures and the velocity at the vertex $M_{q}$. This is the topic of the next paragraph.

\subsection{Evaluation of the velocity and pressures at the vertices.}

Using sufficient conditions (27) and (36) we write the linear system satisfied by the components of the velocity $\boldsymbol{V}_{q}^{\star}$ of an internal vertex $M_{q}$. This generic vertex is not on the boundary of the domain, so that it is surrounded by the cells $\Omega_{k}, k=1 \ldots K(q)$, see Figure 4 . With these new notations, if we shift index $(k \rightarrow k-1)$ in the second term of $(27)$ and get:

$$
\sum_{k=1}^{K(q)} L_{k}\left(P_{q, k}^{\star, k}-P_{q, k}^{\star, k-1}\right) \mathbf{N}_{\boldsymbol{k}}^{\boldsymbol{k}-\mathbf{1}}=\mathbf{0},
$$




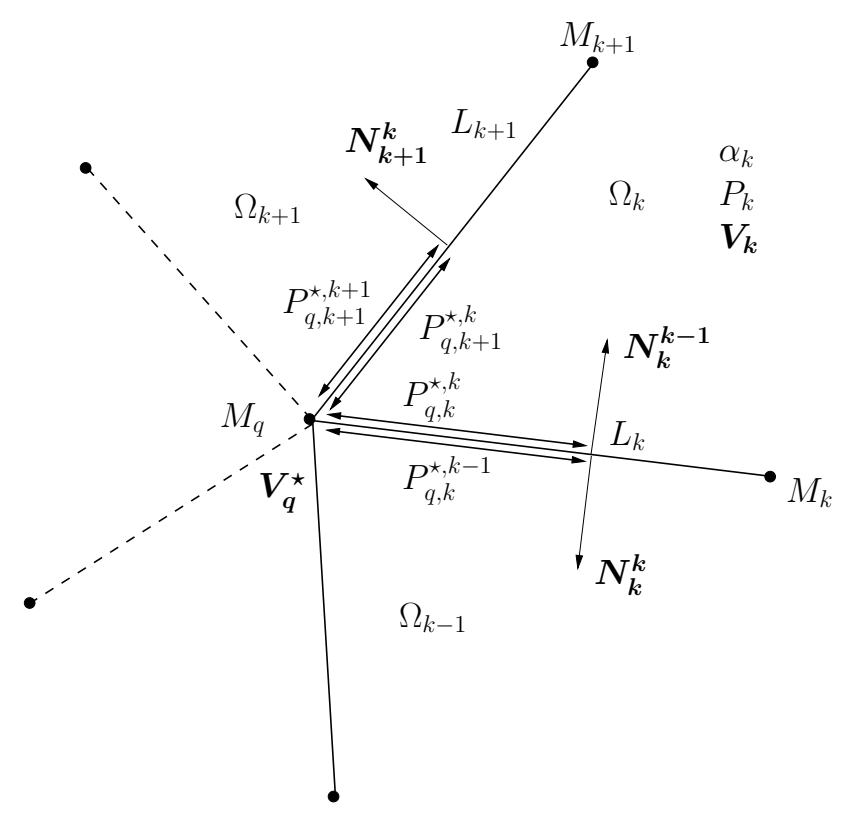

Figure 4: States around internal node $M_{q}$

where we have used $\boldsymbol{N}_{\boldsymbol{k}}^{\boldsymbol{k}}=-\boldsymbol{N}_{\boldsymbol{k}}^{\boldsymbol{k}-\mathbf{1}}$. We proceed in the same way for (36):

$$
\left\{\begin{array}{l}
P_{q, k}^{\star, k}=P_{k}+\alpha_{k}\left(\boldsymbol{V}_{\boldsymbol{q}}^{\star}-\boldsymbol{V}_{\boldsymbol{k}}\right) \cdot \boldsymbol{N}_{\boldsymbol{k}}^{\boldsymbol{k}-\mathbf{1}} \\
P_{q, k}^{\star, k-1}=P_{k-1}-\alpha_{k-1}\left(\boldsymbol{V}_{\boldsymbol{q}}^{\star}-\boldsymbol{V}_{\boldsymbol{k}-\mathbf{1}}\right) \cdot \boldsymbol{N}_{\boldsymbol{k}}^{\boldsymbol{k}-\mathbf{1}}
\end{array}\right.
$$

After substitution of (38) in (37), we obtain the following equation satisfied by $\boldsymbol{V}_{\boldsymbol{q}}^{\star}$

$$
\sum_{k=1}^{K(q)} L_{k}\left(\alpha_{k-1}+\alpha_{k}\right)\left[\boldsymbol{V}_{\boldsymbol{q}}^{\star} \cdot \boldsymbol{N}_{\boldsymbol{k}}^{\boldsymbol{k}-\mathbf{1}}-\frac{P_{k-1}-P_{k}+\alpha_{k-1} \boldsymbol{V}_{\boldsymbol{k}-\mathbf{1}} \cdot \boldsymbol{N}_{\boldsymbol{k}}^{\boldsymbol{k}-\mathbf{1}}+\alpha_{k} \boldsymbol{V}_{\boldsymbol{k}} \cdot \boldsymbol{N}_{\boldsymbol{k}}^{\boldsymbol{k}-\mathbf{1}}}{\alpha_{k-1}+\alpha_{k}}\right] \boldsymbol{N}_{\boldsymbol{k}}^{\boldsymbol{k}-\mathbf{1}}=\mathbf{0}
$$

If we set

$$
\mathcal{V}_{k}^{\star}=\frac{P_{k-1}-P_{k}+\alpha_{k-1} \boldsymbol{V}_{\boldsymbol{k}-\mathbf{1}} \cdot \boldsymbol{N}_{\boldsymbol{k}}^{\boldsymbol{k}-\mathbf{1}}+\alpha_{k} \boldsymbol{V}_{\boldsymbol{k}} \cdot \boldsymbol{N}_{\boldsymbol{k}}^{\boldsymbol{k}-\mathbf{1}}}{\alpha_{k-1}+\alpha_{k}},
$$

$\mathcal{V}_{k}^{\star}$ is the normal velocity given by the classical one dimensional acoustic Riemann solver for face $\left[M_{q}, M_{k}\right]$. With this notation, we can rewrite (39) and give an interesting interpretation of it

$$
\sum_{k=1}^{K(q)} L_{k}\left(\alpha_{k-1}+\alpha_{k}\right)\left[\boldsymbol{V}_{\boldsymbol{q}}^{\star} \cdot \boldsymbol{N}_{\boldsymbol{k}}^{\boldsymbol{k}-\mathbf{1}}-\mathcal{V}_{k}^{\star}\right] \boldsymbol{N}_{\boldsymbol{k}}^{\boldsymbol{k}-\mathbf{1}}=\mathbf{0}
$$

A straightforward calculation shows that the left hand side of this last equation is the gradient of the following quadratic functional

$$
F\left(u_{q}^{\star}, v_{q}^{\star}\right)=\sum_{k=1}^{K(q)} L_{k}\left(\alpha_{k-1}+\alpha_{k}\right)\left[\boldsymbol{V}_{\boldsymbol{q}}^{\star} \cdot \boldsymbol{N}_{\boldsymbol{k}}^{\boldsymbol{k}-\mathbf{1}}-\mathcal{V}_{k}^{\star}\right]^{2},
$$

where $\left(u_{q}^{\star}, v_{q}^{\star}\right)$ denotes the components of $\boldsymbol{V}_{\boldsymbol{q}}^{\star}$. Consequently, the solution of equation (39) reaches the minimum of the functional $F\left(u_{q}^{\star}, v_{q}^{\star}\right)$. Hence, it appears that the nodal velocity $\boldsymbol{V}_{q}^{\star}$ is obtained from a 
weighted least squares procedure. This least squares procedure corresponds to the over-determined system equating the projection of the nodal velocity onto the edge normal $N_{k}^{k-1}$ with the normal velocity $\mathcal{V}_{k}^{\star}$ obtained from the acoustic approximate Riemann solver. For each edge impinging on node $M_{q}$, the weight is $L_{k}\left(\alpha_{k-1}+\alpha_{k}\right)$.

Finally, we write the $2 \times 2$ linear system satisfied by $\left(u_{q}^{\star}, v_{q}^{\star}\right)$ :

$$
\left\{\begin{array}{l}
A u_{q}^{\star}+C v_{q}^{\star}=S M_{X} \\
C u_{q}^{\star}+B v_{q}^{\star}=S M_{Y}
\end{array}\right.
$$

where $A, B$ and $C$ are defined by:

$$
\begin{aligned}
& A=\sum_{k=1}^{K(q)} L_{k}\left(\alpha_{k-1}+\alpha_{k}\right)\left(N_{k, X}^{k-1}\right)^{2}, \\
& B=\sum_{\substack{k=1 \\
K(q)}} L_{k}\left(\alpha_{k-1}+\alpha_{k}\right)\left(N_{k, Y}^{k-1}\right)^{2}, \\
& C=\sum_{k=1}^{K(q)} L_{k}\left(\alpha_{k-1}+\alpha_{k}\right) N_{k, X}^{k-1} N_{k, Y}^{k-1} .
\end{aligned}
$$

Here, $\left(N_{k, X}^{k-1}, N_{k, Y}^{k-1}\right)$ are the coordinates of $\boldsymbol{N}_{k}^{k}$. The right hand side $\left(S M_{X}, S M_{Y}\right)$ of (41) are the components of the vector $\boldsymbol{S} \boldsymbol{M}$ defined by

$$
\boldsymbol{S} \boldsymbol{M}=\sum_{k=1}^{K(q)} L_{k}\left(\alpha_{k-1}+\alpha_{k}\right) \mathcal{V}_{k}^{\star} \boldsymbol{N}_{\boldsymbol{k}}^{\boldsymbol{k - 1}} .
$$

The determinant $\Delta$ of (41) is $\Delta=A B-C^{2}$. We show that it is always positive. In order to simplify notations, let us introduce $\beta_{k}=\sqrt{L_{k}\left(\alpha_{k-1}+\alpha_{k}\right)}$ and $\boldsymbol{N}_{\boldsymbol{k}}=\boldsymbol{N}_{\boldsymbol{k}}^{\boldsymbol{k}-\boldsymbol{1}}$ for $k=1 \ldots K(q)$. We define

$$
\begin{aligned}
& \boldsymbol{U}_{\boldsymbol{X}}=\left(\beta_{1} N_{1, X}, \ldots, \beta_{K} N_{K, X}\right)^{t}, \\
& \boldsymbol{U}_{\boldsymbol{Y}}=\left(\beta_{1} N_{1, Y}, \ldots, \beta_{K} N_{K, Y}\right)^{t} .
\end{aligned}
$$

We have immediately: $A=\left\|\boldsymbol{U}_{\boldsymbol{X}}\right\|^{2}, B=\left\|\boldsymbol{U}_{\boldsymbol{Y}}\right\|^{2}$ and $C=<\boldsymbol{U}_{\boldsymbol{X}}, \boldsymbol{U}_{\boldsymbol{Y}}>$ where $<,>$ is the inner scalar product of $\mathbb{R}^{K}$ and \|\| its associated norm. From the Cauchy-Schwarz inequality, we know that $\Delta \geq 0$. In fact, $\Delta=0$ if and only if one of the two vectors is null or $\boldsymbol{U}_{\boldsymbol{X}}$ and $\boldsymbol{U}_{\boldsymbol{Y}}$ are colinear. This situation is generally impossible unless the edges around the node merge into a single line.

We have shown that the system (41) has always a unique solution which determines the velocity $\boldsymbol{V}_{\boldsymbol{q}}^{\star}$. From (38), we can get the pressures $P_{q, k}^{\star, k}$ and $P_{q, k}^{\star, k-1}$. Moreover, this nodal solver is invariant under translation, homothety and rotation of center $M_{q}$. Before we detail our implementation of the boundary conditions, we provide in the next paragraph an interpretation of our results in the case of a one-dimensional flow with a planar symmetry and in the case of a flow with a cylindrical symmetry.

Remark 3. If the flow is uniform (i.e. with a uniform velocity $\boldsymbol{V}^{0}$ and pressure $P^{0}$ ), we can easily check that the solution of (41) reduces to $\boldsymbol{V}_{q}^{\star}=\boldsymbol{V}^{0}$.

\subsection{Interpretation of the solver in two simple cases}

\subsubsection{One-dimensional flow with planar symmetry}

Let us consider $M_{q}$ surrounded by four quadrangular cells. They are numbered from 1 to 4 in the counter clockwise direction, see Figure 5 . We consider the frame $\left(M_{q}, \boldsymbol{e}_{\boldsymbol{X}}, \boldsymbol{e}_{\boldsymbol{Y}}\right)$. The vector $\boldsymbol{N}_{k}^{k-1}$ is the normal to the edge shared by cells $k-1$ and $k$. The length of this edge is $L_{k}$. The indexing system is periodic with period 4, the flow is one-dimensional in the $\boldsymbol{e}_{X}$ direction so that the velocity field in the cell $k$ reduces to $\boldsymbol{V}_{k}=u_{k} \boldsymbol{e}_{\boldsymbol{X}}$. Moreover, any scalar quantity $\varphi$ satisfies $\varphi_{1} \equiv \varphi_{4}$ and $\varphi_{2} \equiv \varphi_{3}$. The cells 1 and 4 contain 




Figure 5: One-dimensional planar flow

the state defined by $P_{1}, c_{1}, \boldsymbol{V}_{\mathbf{1}}=u_{1} \boldsymbol{e}_{\boldsymbol{X}}$. The cells 2 and 3 contain the state defined by $P_{2}, c_{2}, \boldsymbol{V}_{\mathbf{2}}=u_{2} \boldsymbol{e}_{\boldsymbol{X}}$. Using these notations, the evaluation of the matrix coefficients $A, B, C$ is straightforward

$$
\begin{aligned}
& A=\left(L_{2}+L_{4}\right)\left(\rho_{1} c_{1}+\rho_{2} c_{2}\right), \\
& B=2\left(L_{1} \rho_{1} c_{1}+L_{3} \rho_{2} c_{2}\right), \\
& C=0 .
\end{aligned}
$$

The calculation of $\boldsymbol{S} \boldsymbol{M}$ gives:

$$
\begin{aligned}
& S M_{X}=\left(L_{2}+L_{4}\right)\left(P_{1}-P_{2}+\rho_{1} c_{1} u_{1}+\rho_{2} c_{2} u_{2}\right), \\
& S M_{Y}=0 .
\end{aligned}
$$

Therefore $v_{q}^{\star}=0$ and

$$
u_{q}^{\star}=\frac{P_{1}-P_{2}+\rho_{1} c_{1} u_{1}+\rho_{2} c_{2} u_{2}}{\rho_{1} c_{1}+\rho_{2} c_{2}} .
$$

We recover exactly the acoustic Riemann solver !

\subsubsection{One-dimensional flow with cylindrical symmetry}

We consider an equi-angular cylindrical mesh centered at $O$. We denote by $\theta$ the angle of any sector. The mesh may be non regular in the radial direction. Any vertex $M_{q}$ is surrounded by four trapezoidal cells. In order to simplify the algebra, we choose to work in the local orthonormal frame $\left(M_{q}, \boldsymbol{e}_{\boldsymbol{X}}, \boldsymbol{e}_{\boldsymbol{Y}}\right)$, where $\boldsymbol{e}_{\boldsymbol{X}}$ is the unit vector colinear to $\boldsymbol{O} \boldsymbol{M}_{\boldsymbol{q}}$, see Figure 6 . We use the same conventions as in the previous paragraph. The cells 1 and 4 contain the states defined by $P_{1}, \rho_{1}, c_{1}$. The cells 2 and 3 contains the states defined by $P_{2}, \rho_{2}, c_{2}$. Due to cylindrical symmetry, the velocities are defined by $\boldsymbol{V}_{\mathbf{1}}=V_{1} \boldsymbol{N}_{\mathbf{2}}^{\mathbf{1}}, \boldsymbol{V}_{\mathbf{2}}=V_{2} \boldsymbol{N}_{\mathbf{2}}^{\mathbf{1}}$, $\boldsymbol{V}_{\mathbf{3}}=-V_{2} \boldsymbol{N}_{\mathbf{4}}^{\mathbf{3}}$ and $\boldsymbol{V}_{\mathbf{4}}=-V_{\mathbf{1}} \boldsymbol{N}_{\mathbf{4}}^{\mathbf{3}}$. In the frame $\left(M_{q}, \boldsymbol{e}_{\boldsymbol{X}}, \boldsymbol{e}_{\boldsymbol{Y}}\right)$, the normals write $\boldsymbol{N}_{\mathbf{2}}^{\mathbf{1}}=\left(\begin{array}{c}\cos (\theta / 2) \\ -\sin (\theta / 2)\end{array}\right)$, $\boldsymbol{N}_{\mathbf{2}}^{\mathbf{3}}=\left(\begin{array}{l}0 \\ 1\end{array}\right), \boldsymbol{N}_{\mathbf{4}}^{\mathbf{3}}=\left(\begin{array}{l}-\cos (\theta / 2) \\ -\sin (\theta / 2)\end{array}\right)$ and $\boldsymbol{N}_{\mathbf{1}}^{\mathbf{4}}=\left(\begin{array}{c}0 \\ -1\end{array}\right)$. After calculations we get

$$
\begin{aligned}
A & =\left(L_{2}+L_{4}\right) \cos ^{2}\left(\frac{\theta}{2}\right)\left(\rho_{1} c_{1}+\rho_{2} c_{2}\right), \\
B & =2 L_{1} \rho_{1} c_{1}+\left(L_{2}+L_{4}\right) \sin ^{2}\left(\frac{\theta}{2}\right)\left(\rho_{1} c_{1}+\rho_{2} c_{2}\right)+2 L_{3} \rho_{2} c_{2}, \\
C & =\left(L_{4}-L_{2}\right) \sin \left(\frac{\theta}{2}\right) \cos \left(\frac{\theta}{2}\right)\left(\rho_{1} c_{1}+\rho_{2} c_{2}\right) .
\end{aligned}
$$




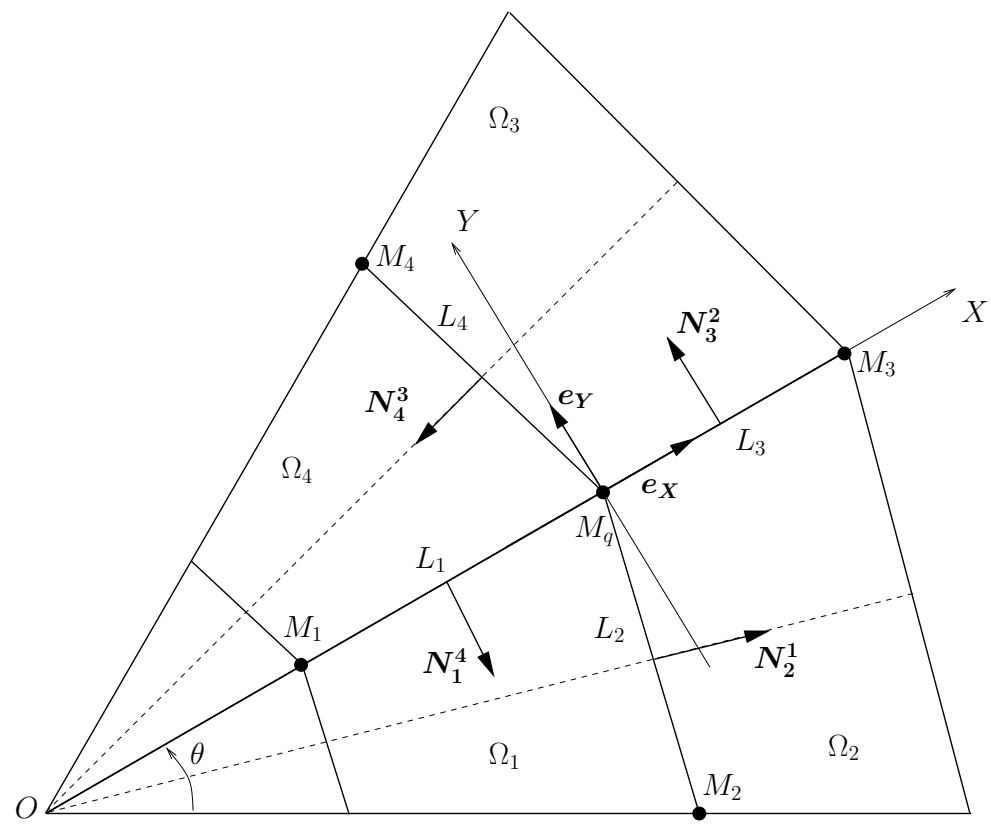

Figure 6: One-dimensional cylindrical flow

We also obtain:

$$
\boldsymbol{S} \boldsymbol{M}=\left(P_{1}-P_{2}+\rho_{1} c_{1} V_{1}+\rho_{2} c_{2} V_{2}\right)\left(\begin{array}{c}
\left(L_{2}+L_{4}\right) \cos (\theta / 2) \\
\left(L_{4}-L_{2}\right) \sin (\theta / 2)
\end{array}\right) .
$$

Using the symmetry we have $L_{2}=L_{4}$, thus $C=0$ and $S M_{Y}=0$. Therefore $v_{q}^{\star}=0$ and the velocity is of the form $\boldsymbol{V}_{\boldsymbol{q}}^{\star}=u_{q}^{\star} \boldsymbol{e}_{\boldsymbol{X}}$. The evaluation of $u_{q}^{\star}$ is straightforward

$$
u_{q}^{\star}=\frac{P_{1}-P_{2}+\rho_{1} c_{1} V_{1}+\rho_{2} c_{2} V_{2}}{\rho_{1} c_{1}+\rho_{2} c_{2}} \frac{1}{\cos \left(\frac{\theta}{2}\right)} .
$$

This is once more the acoustic solver modified by a geometrical factor that is a consequence of the projection of $\boldsymbol{V}_{\boldsymbol{k}}$ on the axis $\left[O, M_{q}\right]$. This term has no consequence since $\cos \left(\frac{\theta}{2}\right) \rightarrow 1$ when $\theta \rightarrow 0$. We also notice that the velocity is radial because the angle between two sectors is uniform. If the mesh does not satisfy this property, therefore $L_{2} \neq L_{4}$ and $C \neq 0$, consequently $v_{q}^{\star} \neq 0$.

\subsection{Boundary conditions}

In this paragraph, we explain our implementation of boundary conditions which is consistent with our internal solver. In the Lagrangian formalism, we have to consider two types of boundary conditions on the boundary $\partial \Omega$ : either we impose the pressure or we impose the normal component of the velocity. We use the same type of notations as in section 4.1. Let $M_{q} \in \partial \Omega$. It is surrounded by $K(q)=K$ cells contained in the domain $\Omega$. There are $K+1$ edges impinging on $M_{q}$. They are numbered counter clockwise, see Figure 7.The first and last cells $\Omega_{1}$ and $\Omega_{K}$ have an edge on the boundary $\partial \Omega$. The outward normals to the two boundary edges coming out of $M_{q}$ are denoted by $-N_{1}^{0}$ and $N_{K+1}^{K}$ coherently with our notations.

\subsubsection{Case of a prescribed pressure}

We denote by $\Pi_{1}^{\star}$ and $\Pi_{K+1}^{\star}$ the pressure that are imposed on the boundary edges, see Figure 7 . We have to compute the $2 K$ pressures $P_{q, k}^{\star, k-1} ; k=2 \ldots K+1$ and $P_{q, k}^{\star, k} ; k=1 \ldots K$, adding the velocity $\boldsymbol{V}_{\boldsymbol{q}}^{\star}$ of 


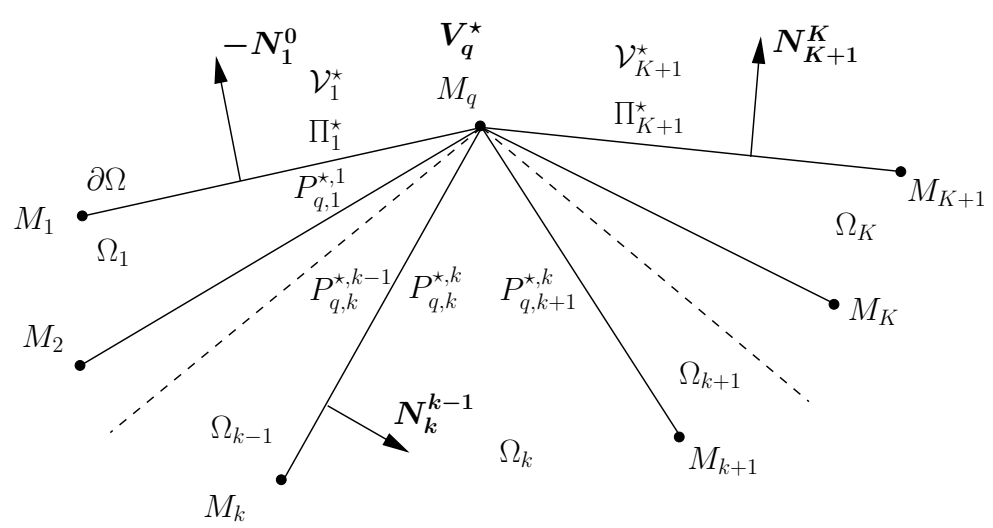

Figure 7: Notations for the boundary conditions

the vertex $M_{q}$, we have a total number of $2 K+2$ scalar unknowns. These unknowns satisfy the previous relations (conservation of momentum, entropy inequality). For the conservation relation, we make a balance around the vertex $M_{q}$ by counting the pressure forces due to the boundary conditions. We get

$$
\begin{gathered}
-L_{1} P_{q, 1}^{\star, 1} \boldsymbol{N}_{\mathbf{1}}^{\mathbf{0}}+\sum_{k=2}^{K} L_{k}\left(P_{q, k}^{\star, k-1}-P_{q, k}^{\star, k}\right) \boldsymbol{N}_{\boldsymbol{k}}^{\boldsymbol{k}-\mathbf{1}}+L_{K+1} P_{q, K+1}^{\star, K} \boldsymbol{N}_{\boldsymbol{K}+\mathbf{1}}^{\boldsymbol{K}}= \\
-L_{1} \Pi_{1}^{\star} \boldsymbol{N}_{\mathbf{1}}^{\mathbf{0}}+L_{K+1} \Pi_{K+1}^{\star} \boldsymbol{N}_{\boldsymbol{K}+\mathbf{1}}^{\boldsymbol{K}} .
\end{gathered}
$$

The entropy inequality provides

$$
\begin{aligned}
& P_{q, k}^{\star, k}=P_{k}+\alpha_{k}\left(\boldsymbol{V}_{\boldsymbol{q}}^{\star}-\boldsymbol{V}_{\boldsymbol{k}}\right) \cdot \boldsymbol{N}_{\boldsymbol{k}}^{\boldsymbol{k}-\mathbf{1}}, k=1 \ldots K, \\
& P_{q, k}^{\star, k-1}=P_{k-1}-\alpha_{k-1}\left(\boldsymbol{V}_{\boldsymbol{q}}^{\star}-\boldsymbol{V}_{\boldsymbol{k}-\mathbf{1}}\right) \cdot \boldsymbol{N}_{\boldsymbol{k}}^{\boldsymbol{k}-\mathbf{1}}, k=2 \ldots K+1 .
\end{aligned}
$$

The use of (43) in (42) leads to:

$$
\left\{\begin{array}{l}
\tilde{A} u_{q}^{\star}+\tilde{C} v_{q}^{\star}=\widetilde{S M_{X}}, \\
\tilde{C} u_{q}^{\star}+\tilde{B} v_{q}^{\star}=\widetilde{S M_{Y}}
\end{array}\right.
$$

where the coefficients are

$$
\begin{aligned}
& \tilde{A}=\sum_{k=2}^{K} L_{k}\left(\alpha_{k-1}+\alpha_{k}\right)\left(N_{k, X}^{k-1}\right)^{2}+L_{1} \alpha_{1}\left(N_{1, X}^{0}\right)^{2}+L_{K+1} \alpha_{K+1}\left(N_{K+1, X}^{K}\right)^{2}, \\
& \tilde{B}=\sum_{k=2}^{K} L_{k}\left(\alpha_{k-1}+\alpha_{k}\right)\left(N_{k, Y}^{k-1}\right)^{2}+L_{1} \alpha_{1}\left(N_{1, Y}^{0}\right)^{2}+L_{K+1} \alpha_{K+1}\left(N_{K+1, Y}^{K}\right)^{2}, \\
& \tilde{C}=\sum_{k=2}^{K} L_{k}\left(\alpha_{k-1}+\alpha_{k}\right) N_{k, X}^{k-1} N_{k, Y}^{k-1}+L_{1} \alpha_{1} N_{1, X}^{0} N_{1, Y}^{0}+L_{K+1} \alpha_{K+1} N_{K+1, X}^{K} N_{K+1, Y}^{K} .
\end{aligned}
$$

We see that we recover the coefficients $A, B$ and $C$ where the contribution of the boundary faces are now taken into account. The right hand side of (44) writes

$$
\begin{aligned}
\widetilde{\boldsymbol{S M}} & =\sum_{k=2}^{K} L_{k}\left[P_{k-1}-P_{k}+\left(\alpha_{k-1} \boldsymbol{V}_{\boldsymbol{k}-\mathbf{1}}+\alpha_{k} \boldsymbol{V}_{\boldsymbol{k}}\right) \cdot \boldsymbol{N}_{\boldsymbol{k}-\mathbf{1}}^{\boldsymbol{k}}\right] \boldsymbol{N}_{\boldsymbol{k}-\mathbf{1}}^{\boldsymbol{k}} \\
& -L_{1}\left[P_{1}-\Pi_{1}^{\star}-\alpha_{1} \boldsymbol{V}_{\mathbf{1}} \cdot \boldsymbol{N}_{\mathbf{1}}^{\mathbf{0}}\right] \boldsymbol{N}_{\mathbf{1}}^{\mathbf{0}}+L_{K+1}\left[P_{K}-\Pi_{K+1}^{\star}+\alpha_{K} \boldsymbol{V}_{\boldsymbol{K}} \cdot \boldsymbol{N}_{\boldsymbol{K}}^{\boldsymbol{K}+\mathbf{1}}\right] \boldsymbol{N}_{\boldsymbol{K}}^{\boldsymbol{K}+\mathbf{1}} .
\end{aligned}
$$


From the Cauchy Schwartz inequality, we know that $\tilde{C}^{2}<\tilde{A} \tilde{B}$. Hence, the linear system (44) has always a unique solution $\left(u_{q}^{\star}, v_{q}^{\star}\right)$. The pressures $P_{q, k}^{\star, k-1} ; k=2 \ldots K+1$ and $P_{q, k}^{\star, k} ; k=1 \ldots K$ are obtained from (43).

\subsubsection{Case of a prescribed normal velocity}

Let $\mathcal{V}_{1}^{\star}$ and $\mathcal{V}_{K+1}^{\star}$ be the values of the prescribed normal velocities on the boundary edges coming out of $M_{q}$. We distinguish the two following cases:

- $-\boldsymbol{N}_{\mathbf{1}}^{\mathbf{0}}$ and $\boldsymbol{N}_{\boldsymbol{K}}^{\boldsymbol{K}+\mathbf{1}}$ are not colinear: in this case the value of $\boldsymbol{V}_{\boldsymbol{q}}^{\star}$ is given by the boundary conditions and the components of the vertex velocity are solution of the linear system:

$$
\left\{\begin{array}{l}
-N_{1, X}^{0} u_{q}^{\star}-N_{1, Y}^{0} v_{q}^{\star}=\mathcal{V}_{1}^{\star} \\
N_{K, X}^{K+1} u_{q}^{\star}+N_{K, Y}^{K+1} v_{q}^{\star}=\mathcal{V}_{K+1}^{\star}
\end{array}\right.
$$

This linear system has always a unique solution, since the normals are not colinear, the determinant is non zero. The pressures are computed from (43).

- $-\boldsymbol{N}_{\mathbf{1}}^{\mathbf{0}}$ and $\boldsymbol{N}_{\boldsymbol{K}}^{\boldsymbol{K}+\mathbf{1}}$ are colinear: in this case, $\boldsymbol{V}_{\boldsymbol{q}}^{\star}$ is not given directly, and we need to know the balance of momentum around the vertex $M_{q}$ which takes the boundary conditions into account:

$$
\begin{gathered}
-L_{1} P_{q, 1}^{\star, 1} \boldsymbol{N}_{\mathbf{1}}^{\mathbf{0}}+\sum_{k=2}^{K} L_{k}\left(P_{q, k}^{\star, k-1}-P_{q, k}^{\star, k}\right) \boldsymbol{N}_{\boldsymbol{k}}^{\boldsymbol{k}-\mathbf{1}}+L_{K+1} P_{q, K+1}^{\star, K} \boldsymbol{N}_{\boldsymbol{K}+\mathbf{1}}^{\boldsymbol{K}}= \\
\left(-L_{1} \boldsymbol{N}_{\mathbf{1}}^{\mathbf{0}}+L_{K+1} \boldsymbol{N}_{\boldsymbol{K}}^{\boldsymbol{K}+\mathbf{1}}\right) \Pi^{\star}
\end{gathered}
$$

where $\Pi^{\star}$ is an average pressure applied on the external side of the edges coming out of $M_{q}$. The pressure $\Pi^{\star}$ is a new unknown, but we have an additional equation corresponding to the boundary condition

$$
\left(-L_{1} \boldsymbol{N}_{\mathbf{1}}^{\mathbf{0}}+L_{K+1} \boldsymbol{N}_{\boldsymbol{K}}^{\boldsymbol{K}+\mathbf{1}}\right) \cdot \boldsymbol{V}_{\boldsymbol{q}}^{\star}=L_{1} \mathcal{V}_{1}^{\star}+L_{K+1} \mathcal{V}_{K+1}^{\star}
$$

Using (43) in (45) combined with (46), we get

$$
\left\{\begin{array}{c}
\tilde{A} u_{q}^{\star}+\tilde{C} v_{q}^{\star}+D \Pi^{\star}=\widehat{S M_{X}}, \\
\tilde{C} u_{q}^{\star}+\tilde{B} v_{q}^{\star}+E \Pi^{\star}=\widehat{S M_{Y}} \\
D u_{q}^{\star}+E v_{q}^{\star}=L_{1} \mathcal{V}_{1}^{\star}+L_{K+1} \mathcal{V}_{K+1}^{\star}
\end{array}\right.
$$

The coefficients $\tilde{A}, \tilde{B}$ and $\tilde{C}$ have already been defined in the previous paragraph and we have set $D=-L_{1} N_{1, X}^{0}+L_{K+1} N_{K, X}^{K+1}, E=-L_{1} N_{1, Y}^{0}+L_{K+1} N_{K, Y}^{K+1}$. The right hand side of (47) is

$$
\begin{aligned}
\widehat{\boldsymbol{S M}} & =\sum_{k=2}^{K} L_{k}\left[P_{k-1}-P_{k}+\left(\alpha_{k-1} \boldsymbol{V}_{\boldsymbol{k}-\mathbf{1}}+\alpha_{k} \boldsymbol{V}_{\boldsymbol{k}}\right) \cdot \boldsymbol{N}_{\boldsymbol{k}-\mathbf{1}}^{\boldsymbol{k}}\right] \boldsymbol{N}_{\boldsymbol{k}-\mathbf{1}}^{\boldsymbol{k}} \\
& -L_{1}\left[P_{1}-\alpha_{1} \boldsymbol{V}_{\mathbf{1}} \cdot \boldsymbol{N}_{\mathbf{1}}^{\mathbf{0}}\right] \boldsymbol{N}_{\mathbf{1}}^{\mathbf{0}}+L_{K+1}\left[P_{K}+\alpha_{K} \boldsymbol{V}_{\boldsymbol{K}} \cdot \boldsymbol{N}_{\boldsymbol{K}}^{\boldsymbol{K}+\mathbf{1}}\right] \boldsymbol{N}_{\boldsymbol{K}}^{\boldsymbol{K}+\mathbf{1}}
\end{aligned}
$$

The determinant of (47) is $\Delta=-\tilde{A} E+2 \tilde{C} D E-\tilde{B} D^{2}$. Using the fact that $|\tilde{C}|<\sqrt{\tilde{A} \tilde{B}}$, one can show that $\Delta<0$ provided the mesh is not degenerated: (47) always admits a unique solution $\left(u_{q}^{\star}, v_{q}^{\star}\right)$, and then we can always define a unique set of pressure due to (43).

\subsection{Some remarks}

From conservation arguments and one entropy inequality, we have been able to construct a nodal solver. This scheme shares some characteristics with the method developed in [9], it can be interpreted as a twodimensional extension of the acoustic solver. It is interesting to realize that we only need the knowledge of 
the isentropic speed of sound: it is very easy to extend it to more general equation of state. The precise form of the equation of state, analytical or tabulated, does not matter.

We have rigorously shown that the linear system which provides the components of the vertex velocity admits a unique solution, provided that the mesh is not degenerated, see the end of section 4.4. This is also true for vertex located on the boundary, whereas with the method developed in [9] some difficulties have been encountered for vertex located at the corner on the boundary of a rectangular domain.

We have also checked that our solver recovers the one-dimensional acoustic solver in the case of onedimensional planar and cylindrical flows, contrarily to [9]. More precisely, the calculation of the node velocity with the solver described in [9], for a one dimensional flow with planar symmetry gives

$$
u_{q}^{\star}=\frac{P_{1}-P_{2}+\rho_{1} c_{1} u_{1}+\rho_{2} c_{2} u_{2}}{\rho_{1} c_{1}+\rho_{2} c_{2}} \frac{\sqrt{\Delta X^{2}+\Delta Y^{2}}}{\Delta Y},
$$

where $L_{1}=L_{3}=\Delta X$ and $L_{2}=L_{4}=\Delta Y$ are mesh spacing along directions $\boldsymbol{e}_{\boldsymbol{X}}$ and $\boldsymbol{e}_{\boldsymbol{Y}}$. With this nodal solver, one recovers the one dimensional acoustic solver velocity multiplied by a factor which depends on the aspect ratio of the cells.

Last, our method fully answers the questions raised at the beginning of this paper since it uniquely provides the vertex velocity and the face fluxes. The main new features of our scheme is the introduction of four pressures on each edge, two for each node on each side of the edge, that is, eight pressures at each node for a quadrangular mesh.

We can compute, in a coherent way, the vertex motion as well as the face fluxes. This solver has been constructed delocalizing the fluxes from the faces to the vertices. Consequently, the conservation of momentum and energy are obtained around the node. This implies a fundamental difference with a standard finite volume scheme.

This difference can be illustrated by the consideration of a mesh made of quadrangular cells. We denote by $1,2,3$ and 4 the vertices of the cell $\Omega_{i}$ and $S, S E, E, N E, N, N W, O$ et $S W$ the neighboring cells, see Figure 8. In a standard finite volume scheme with an approximate Riemann solver, the cell $\Omega_{i}$ only



Figure 8: Stencil for a quadrangular mesh

exchanges information with the neighboring cells having a common face, i.e. the cells $S, E, N$ and $O$. This is a 5 point scheme.

With our scheme, the cell $\Omega_{i}$ exchanges momentum and energy with the neighboring cells having a common vertex, i.e. the cells $S, S E, E, N E, N, N W, W$ and $S W$. This is a 9 point scheme. The four additional cells reinforce the genuinely multidimensional nature of our scheme. 


\section{Time discretization}

In this section, we time discretize the system that describes the evolution of the physical variables $\left(\tau_{i}, \boldsymbol{V}_{\boldsymbol{i}}, E_{i}\right)$ in the cell $\Omega_{i}$

$$
\begin{aligned}
& m_{i} \frac{d}{d t} \tau_{i}-\sum_{r=1}^{R(i)} L_{r, r+1} \boldsymbol{V}_{\boldsymbol{r}, \boldsymbol{r}+\mathbf{1}}^{\star} \cdot \boldsymbol{N}_{\boldsymbol{r}, \boldsymbol{r}+\mathbf{1}}=0, \\
& m_{i} \frac{d}{d t} \boldsymbol{V}_{\boldsymbol{i}}+\sum_{r=1}^{R(i)} L_{r, r+1} P_{r, r+1}^{\star} \boldsymbol{N}_{\boldsymbol{r}, \boldsymbol{r}+\mathbf{1}}=\mathbf{0}, \\
& m_{i} \frac{d}{d t} E_{i}+\sum_{r=1}^{R(i)} L_{r, r+1}(P \boldsymbol{V})_{r, r+1}^{\star} \cdot \boldsymbol{N}_{\boldsymbol{r}, \boldsymbol{r}+\mathbf{1}}=0,
\end{aligned}
$$

where the face fluxes $\boldsymbol{V}_{\boldsymbol{r}, \boldsymbol{r}+\mathbf{1}}^{\star}, P_{r, r+1}^{\star}$ are evaluated by our solver. For the time discretization of (48) we use a classical forward Euler scheme. A more sophisticated time discretization is not required here, since the scheme is only first order accurate in space. However time discretization of (48) should be done carefully so that all the properties of the semi-discrete system are kept. We list them:

- the variation of volume is coherent with the vertex motion,

- the conservation of momentum and total energy,

- the scheme satisfies an entropy inequality.

The first two properties impose an explicit time discretization, the third one would impose an implicit discretization. We choose an explicit time discretization, knowing that the entropy inequality will be $a$ priori satisfied under a CFL type condition.

We assume to know the physical properties in the cell $\Omega_{i}$ and the geometrical characteristics of the cell at the beginning of the time step $t_{n}$, i.e. $\tau_{i}^{n}, \boldsymbol{V}_{i}^{n}, E_{i}^{n}, P_{i}^{n}, X_{r}^{n}$ and $Y_{r}^{n}$. We are going to compute their values at $t^{n+1}$, and we set $\Delta t=t^{n+1}-t^{n}$.

\subsection{Mesh motion and variation of volume}

The nodal solver provides the vertex velocities $\boldsymbol{V}_{\boldsymbol{r}}^{\star}$ and the face pressures at $P_{r, r+\frac{1}{2}}^{\star, i}, P_{r+\frac{1}{2}, r+1}^{\star, i}$ from the physical variables and geometry characteristics evaluated at time $t^{n}$. The explicit time integration of the trajectory equation provides the location of vertices for any time $t \in\left[t^{n}, t^{n+1}\right]$ :

$$
\begin{aligned}
& X_{r}(t)=X_{r}^{n}+\left(t-t^{n}\right) u_{r}^{\star}, \\
& Y_{r}(t)=Y_{r}^{n}+\left(t-t^{n}\right) v_{r}^{\star},
\end{aligned}
$$

by this way, one gets the location of the vertices at time $t^{n+1}$

$$
\begin{aligned}
& X_{r}^{n+1}=X_{r}^{n}+u_{r}^{\star} \Delta t, \\
& Y_{r}^{n+1}=Y_{r}^{n}+v_{r}^{\star} \Delta t .
\end{aligned}
$$

From this we deduce that $\left(L_{r, r+1} \boldsymbol{N}_{r, \boldsymbol{r}+\mathbf{1}}\right)(t)=\left[Y_{r+1}(t)-Y_{r}(t),-\left(X_{r+1}(t)-X_{r}(t)\right)\right]^{t}$ is linear in time, so that

$$
\int_{t^{n}}^{t^{n+1}}\left(L_{r, r+1} \boldsymbol{N}_{\boldsymbol{r}, \boldsymbol{r + 1}}\right)(t) \mathrm{d} t=\frac{\Delta t}{2}\left[\left(L_{r, r+1} \boldsymbol{N}_{\boldsymbol{r}, \boldsymbol{r + 1}}\right)^{n}+\left(L_{r, r+1} \boldsymbol{N}_{\boldsymbol{r}, \boldsymbol{r + 1}}\right)^{n+1}\right] .
$$


This last results enables to write an approximation of the volume equation which is coherent with the mesh motion, namely

$$
m_{i}\left(\tau_{i}^{n+1}-\tau_{i}^{n}\right)-\frac{\Delta t}{4} \sum_{r=1}^{R(i)}\left[\left(L_{r, r+1} \boldsymbol{N}_{\boldsymbol{r}, \boldsymbol{r}+\mathbf{1}}\right)^{n}+\left(L_{r, r+1} \boldsymbol{N}_{\boldsymbol{r}, \boldsymbol{r}+\mathbf{1}}\right)^{n+1}\right] \cdot\left(\boldsymbol{V}_{\boldsymbol{r}}^{\star}+\boldsymbol{V}_{\boldsymbol{r}+\mathbf{1}}^{\star}\right)=0,
$$

Remark 4. We point out that the specific volume may be updated directly from the ratio of the Lagrangian mass of the cell and the area obtained from the geometry of the cell:

$$
\tau_{i}^{n+1}=\frac{\mathcal{A}_{i}^{n+1}}{m_{i}}
$$

where $\mathcal{A}_{i}^{n+1}$ is the area of $\Omega_{i}$ computed from (50). Here, we have chosen to solve (52) in order to put the stress on the fact that our solver ensures the coherence between the face fluxes and the node motion.

\subsection{Momentum and total energy}

The approximation of the momentum and the total energy equation is fully explicit in order to conserve exactly the momentum and the total energy. The lengths (of edges) and the normals have the same definition as previously. We get

$$
m_{i}\left(\boldsymbol{V}_{\boldsymbol{i}}^{\boldsymbol{n + 1}}-\boldsymbol{V}_{\boldsymbol{i}}^{\boldsymbol{n}}\right)+\frac{\Delta t}{2} \sum_{r=1}^{R(i)} L_{r, r+1}^{n}\left(P_{r, r+\frac{1}{2}}^{\star, i}+P_{r+\frac{1}{2}, r+1}^{\star, i}\right) \boldsymbol{N}_{\boldsymbol{r}, \boldsymbol{r}+\mathbf{1}}^{\boldsymbol{n}}=\mathbf{0}
$$

and for the total energy

$$
m_{i}\left(E_{i}^{n+1}-E_{i}^{n}\right)+\frac{\Delta t}{2} \sum_{r=1}^{R(i)} L_{r, r+1}^{n}\left(P_{r, r+\frac{1}{2}}^{\star, i} \boldsymbol{V}_{\boldsymbol{r}}^{\star}+P_{r+\frac{1}{2}, r+1}^{\star, i} \boldsymbol{V}_{\boldsymbol{r}+\mathbf{1}}^{\star}\right) \cdot \boldsymbol{N}_{\boldsymbol{r}, \boldsymbol{r}+\mathbf{1}}^{\boldsymbol{n}}=0 .
$$

\subsection{Time step limitation}

The time step is evaluated following two criteria. The first one is a standard CFL criterion which guaranties heuristically the monotone behavior of the entropy. The second is more intuitive, but reveals very useful in practice: we limit the variation of the volume of cells over one time step.

\subsubsection{CFL criterion}

We propose a CFL like criterion in order to ensure a positive entropy production in cell $\Omega_{i}$ during the time step. At time $t_{n}$, for each cell $\Omega_{i}$ we denote by $\lambda_{i}^{n}$ the minimal value of the distance between two nodes of the cell. We define

$$
\Delta t_{E}=C_{E} \min _{i=1, \ldots, I} \frac{\lambda_{i}^{n}}{c_{i}^{n}}
$$

where $C_{E}$ is a strictly positive coefficient and $c_{i}$ is the sound speed in the cell. The coefficient $C_{E}$ is computed heuristically and we provide no rigorous analysis which allows such formula. However, extensive numerical experiments show that $C_{E}=0.3$ is a value which provides good numerical results. We have also checked that this value is compatible with a monotone behavior of entropy. The rigorous derivation of this criterion could be obtained by computing the time step which ensures a positive entropy production in cell $\Omega_{i}$ from time $t^{n}$ to $t^{n+1}$. 


\subsubsection{Criterion on the variation of volume}

Using (49), we can compute the area of the cell $i$ at any time $t \in\left[t^{n}, t^{n+1}\right]$. Indeed, we have

$$
\mathcal{A}_{i}(t)=\frac{1}{2} \sum_{r=1}^{R(i)}\left[X_{r}^{n}+\left(t-t^{n}\right) u_{r}^{\star}\right]\left[Y_{r+1}^{n}+\left(t-t^{n}\right) v_{r+1}^{\star}\right]-\left[Y_{r}^{n}+\left(t-t^{n}\right) v_{r}^{\star}\right]\left[X_{r+1}^{n}+\left(t-t^{n}\right) u_{r+1}^{\star}\right] .
$$

Simple algebra gives

$$
\frac{d}{d t} \mathcal{A}_{i}\left(t^{n}\right)=\frac{1}{2} \sum_{r=1}^{R(i)}\left(u_{r}^{\star} Y_{r+1}^{n}+v_{r+1}^{\star} X_{r}^{n}-u_{r+1}^{\star} Y_{r}^{n}-v_{r}^{\star} X_{r+1}^{n}\right) .
$$

After a Taylor expansion we estimate the area at $t=t^{n+1}$ by

$$
\mathcal{A}_{i}^{n+1}=\mathcal{A}_{i}^{n}+\frac{d}{d t} \mathcal{A}_{i}\left(t^{n}\right) \Delta t .
$$

Let $C_{V}$ be a strictly positive coefficient, $\left.C_{V} \in\right] 0,1[$. We look for $\Delta t$ such that

$$
\frac{\left|\mathcal{A}_{i}^{n+1}-\mathcal{A}_{i}^{n}\right|}{\mathcal{A}_{i}^{n}} \leq C_{V} .
$$

To do so, we define

$$
\Delta t_{V}=C_{V} \min _{i=1, \ldots, I}\left\{\frac{\mathcal{A}_{i}^{n}}{\left|\frac{d}{d t} \mathcal{A}_{i}\left(t^{n}\right)\right|}\right\} .
$$

For numerical applications, we choose $C_{V}=0.1$.

Last, the estimation of the next time step $\Delta t^{n+1}$ is given by

$$
\Delta t^{n+1}=\min \left(\Delta t_{E}, \Delta t_{V}, C_{M} \Delta t^{n}\right),
$$

where $\Delta t^{n}$ is the current time step and $C_{M}$ is a multiplicative coefficient which allows the time step to increase. We generally set $C_{M}=1.01$.

\subsection{Description of the algorithm}

\section{Initialization}

At time $t=t^{n}$ we know in each cells $\Omega_{i}, i=1, \ldots, I$ the fluid variables: $\tau_{i}^{n}, \boldsymbol{V}_{i}^{n}, E_{i}^{n}, \rho_{i}^{n}, c_{i}^{n}, P_{i}^{n}$ and the geometrical characteristics: $X_{r}^{n}, Y_{r}^{n}, L_{r, r+1}^{n}, N_{r, r+1}^{n}$ for $r=1, \ldots, R(i)$.

\section{Nodal solver}

- For each internal vertex $M_{q}, q=1, \ldots Q$, we first compute the velocity $\boldsymbol{V}_{\boldsymbol{q}}^{\star}$ by solving the linear system (41) then we evaluate the edge pressures $P_{q, k}^{\star, k}$ and $P_{q, k}^{\star, k-1}$ with (38), for each edge impinging on $M_{q}$.

- For each boundary vertex, we first compute the velocity $\boldsymbol{V}_{\boldsymbol{q}}^{\star}$ by solving (44) or (47), then we calculate the edge pressures $P_{q, k}^{\star, k}$ and $P_{q, k}^{\star, k-1}$ using (43).

\section{Time step limitations}

We compute $\Delta t_{V}$ and $\Delta t_{E}$, then we predict $\Delta t^{n+1}$ from $\Delta t^{n+1}=\min \left(\Delta t_{E}, \Delta t_{V}, C_{M} \Delta t^{n}\right)$.

4. Update of the geometrical quantities

We compute $X_{r}^{n+1}$ and $Y_{r}^{n+1}$ from (50), then we deduce $L_{r, r+1}^{n+1}$ and $\boldsymbol{N}_{r, \boldsymbol{r}+\mathbf{1}}^{\boldsymbol{n}+1}$. 


\section{Update of the physical variables}

We compute the face fluxes from the vertex velocity and the edge pressures, then we get $\tau_{i}^{n+1}, \boldsymbol{V}_{\boldsymbol{i}}^{\boldsymbol{n}+\mathbf{1}}$ and $E_{i}^{n+1}$ from (52),(53) and (54).

\section{Equation of state}

The internal energy is given by $\varepsilon_{i}^{n+1}=E_{i}^{n+1}-\frac{1}{2}\left\|\boldsymbol{V}_{\boldsymbol{i}}^{\boldsymbol{n}+\mathbf{1}}\right\|^{2}$, then we get the pressure $P_{i}^{n+1}$ and the isentropic sound speed $c_{i}$ from the equation of state.

\section{$6 \quad$ Numerical results}

In this section, we present several test cases in order to validate our numerical scheme. For each case we use a perfect gas equation of state, namely $P=(\gamma-1) \rho \varepsilon$ where $\gamma$ is the adiabatic index. We begin by several classical one dimensional test cases characterized by the presence of shock waves of various strengths, rarefaction waves and contact discontinuities. We also provide an isentropic compression. For all these test cases an analytical solution is available so that we can make accurate comparisons. Then, we give an original test representative of the domain of hydrodynamic instabilities. The aim of this test is to show the ability of our scheme to reproduce the growth of such instabilities in the linear regime. Last, we propose a two-dimensional test case which consists in imploding a double layer cylindrical shell under an anisotropic pressure load.

\subsection{Multi-material Sod's shock tube problem}

We are concerned with a multi-material variant of the Sod's shock tube problem defined in [26]. We consider a shock tube of unity length. At the initial time, the states on the left and the right sides of $x=0.5$ are constant. The left state is a high pressure fluid characterized by $\left(\rho_{l}, P_{l}, u_{l}\right)=(1,1,0)$, the right state is a low pressure fluid defined by $\left(\rho_{r}, P_{r}, u_{r}\right)=(0.125,0.1,0)$. The equation of state is defined on the left by $\gamma_{l}=\frac{7}{5}$ and $\gamma_{r}=\frac{5}{3}$ on the right. The computational domain is defined by $(x, y) \in[0,1] \times[0,0.1]$. The

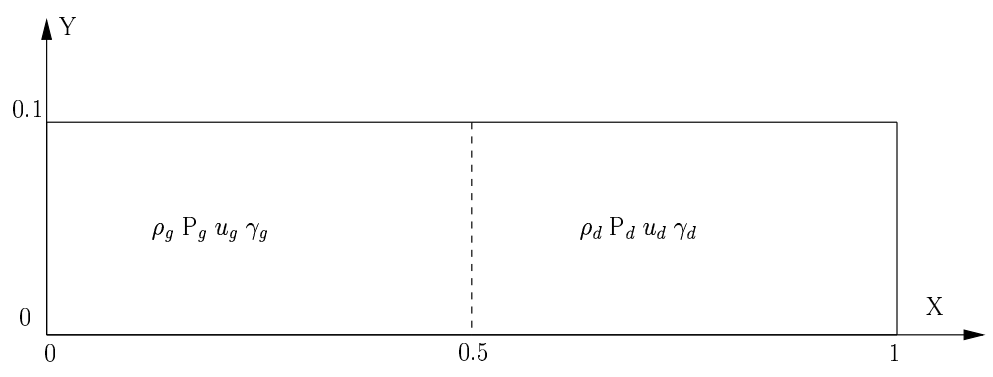

Figure 9: Initial data for the Sod shock tube test case

initial mesh is a cartesian grid, we denote by $n_{x}$ and $n_{y}$ the number of cells in the $x$ and $y$ directions. The boundary conditions are wall boundary conditions: the normal velocity is set to zero. Three simulations are done with $n_{y}=10$ and $n_{x}=100, n_{x}=200$ and finally $n_{x}=400$ in order to check the mesh convergence of the scheme. The time step is evaluated following (55). On Figure 10, we compare the profiles of the physical quantities at $t=0.2$ with the analytical ones for three different meshes. The profiles are similar to what could be obtained with a one dimensional first order Lagrangian scheme based on the acoustic solver, see the interpretation of our scheme in section 4.5.1. The shock is smeared on several cells and the fan is correctly described for a first order scheme. The contact discontinuity is resolved in several cells, this is a consequence of the entropic nature of the solver. We notice an undershoot for the density profile and an overshoot for the internal energy profile which are classical for Lagrangian methods. As expected, the finer the mesh is, the better the results are. 

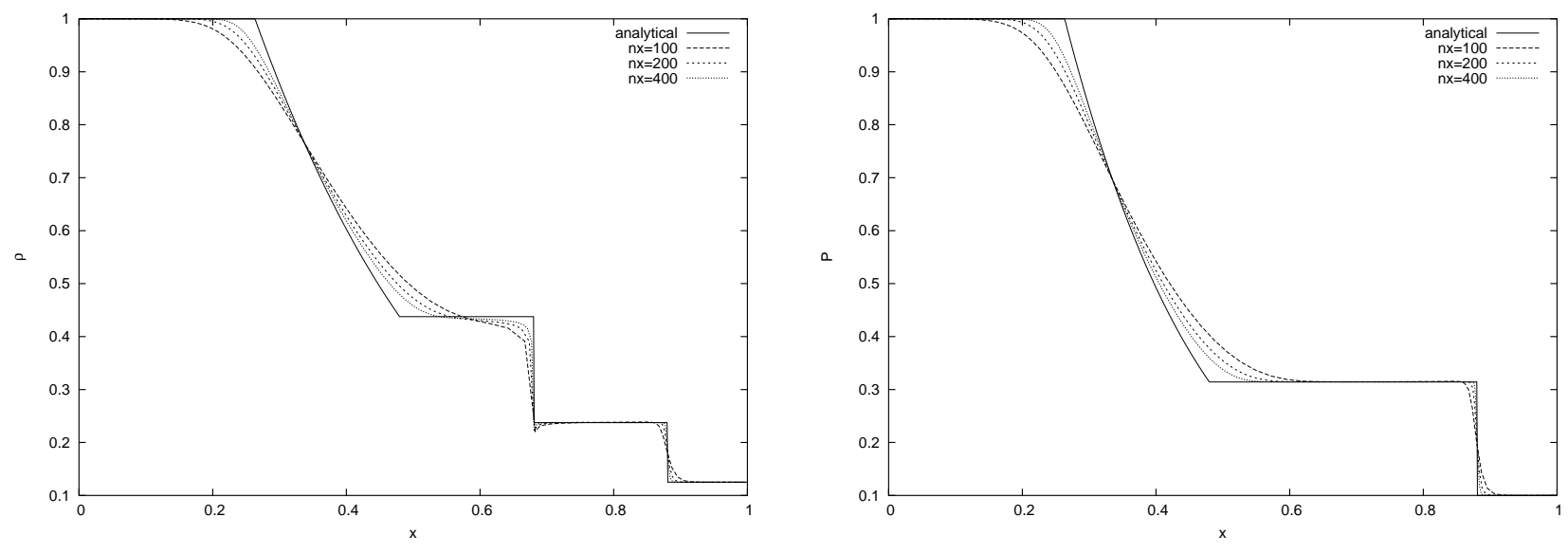

density


Figure 10: Cross-section at $t=0.2$, comparison with the analytical solution 
Remark 5. The resolution of the contact discontinuity and the fan is improved if we use a mesh where the space discretization is adapted, see Figure 11. The adaptation principle is to distribute mass as uniformly as possible throughout the mesh: if $\Delta x_{l}$ (resp. $\Delta x_{r}$ ) is the space discretization parameter on the left (resp. the right) of the initial interface, we evaluate $\Delta x_{l}$ by $\Delta x_{l}=\frac{\rho_{r}}{\rho_{l}} \Delta x_{r}$.

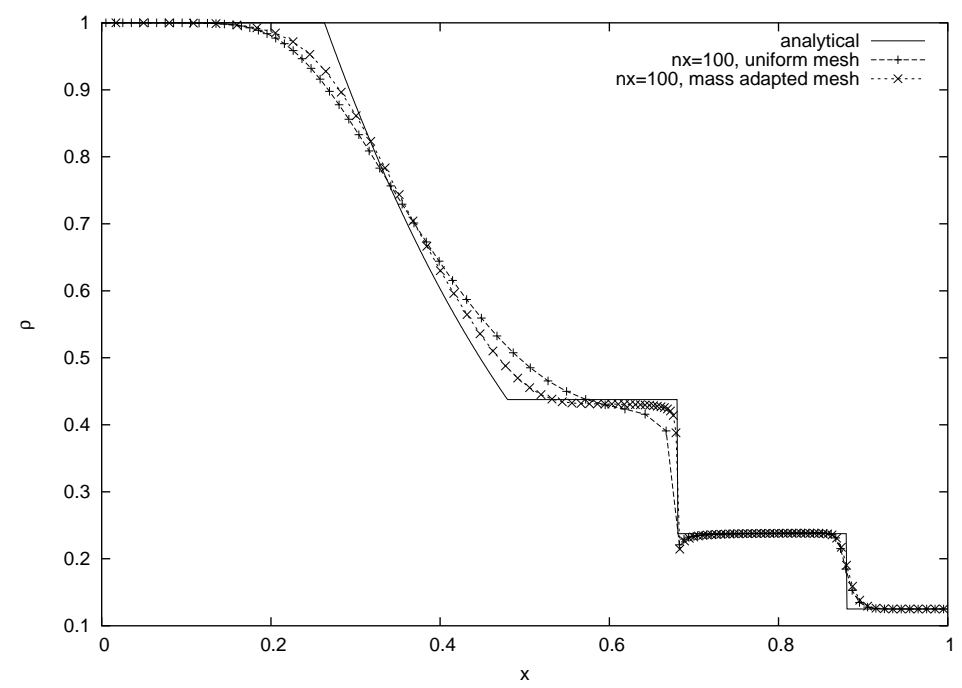

Figure 11: Cross-section of the density for the Sod shock tube at $t=0.2$. The mesh is adapted according to the mass distribution 


\subsection{Noh's test case}

Noh's test case is the implosion of a cylinder of unit radius. The cylinder is filled with a monoatomic perfect gas $(\gamma=5 / 3)$. The initial state is $\left(\rho^{0}, P^{0}, \boldsymbol{V}^{\mathbf{0}}\right)=\left(1,0,-\boldsymbol{e}_{\boldsymbol{R}}\right)$, where $\boldsymbol{e}_{R}$ represents the radial unit vector. This case, defined by Noh in [23], admits a self-similar solution: a shock wave moves inwards at the constant speed $D=1 / 3$. The symmetry of the problem enables to limit the computational domain to an angular sector of angle $\Theta$, see Figure 12. The mesh consists of regular angular sectors, the domain is meshed with triangles near the center and with quadrangles elsewhere. This enables to respect the radial symmetry of the solution. We denote by $n_{x}$ (resp. $n_{y}$ ) the number of layers (resp. angular sectors). In what follows, $\theta=\frac{\Theta}{n_{y}}$. The test case is initialized with $P^{0}=10^{-6}$. The boundary conditions are wall conditions on the two external edges (hence zero normal velocity) and we impose a constant pressure $P^{\star}=P^{0}$ on the external radius $R=1$. The time step is computed following the condition (55). On Figure 13 we have represented the results at

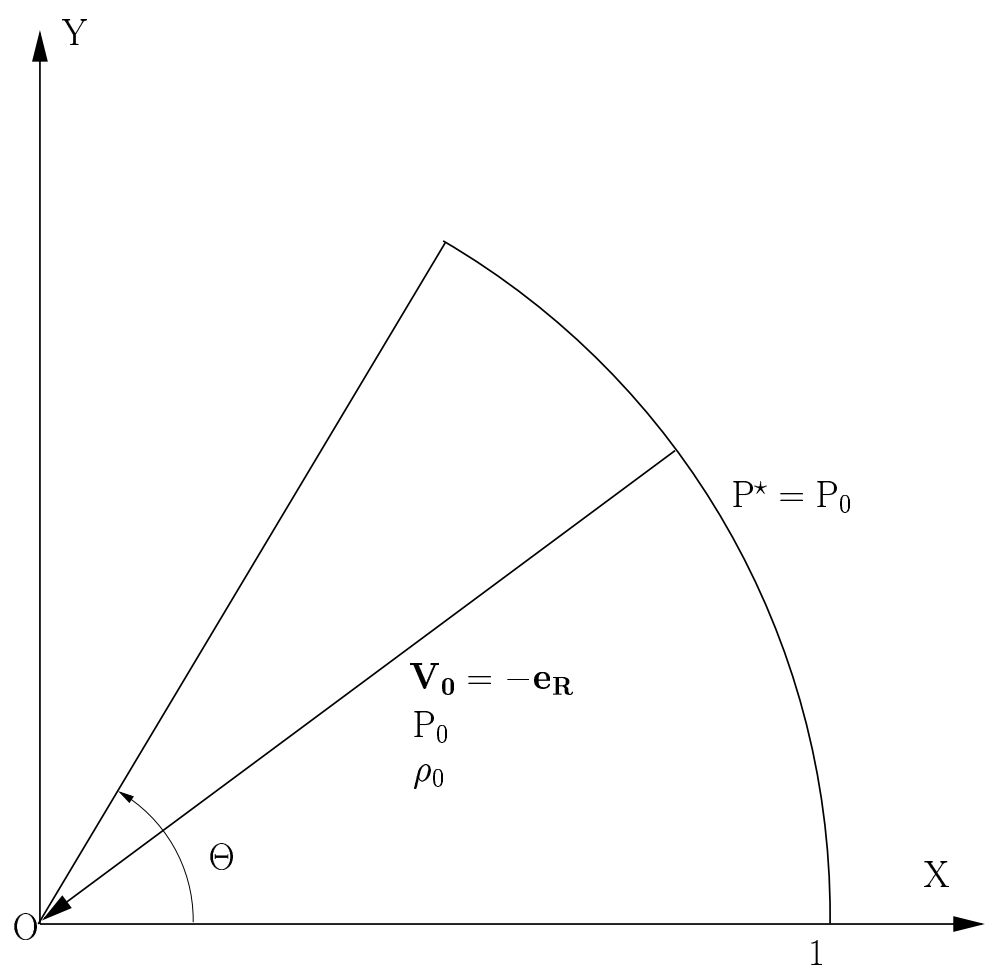

Figure 12: Noh's case: initial conditions

time $t=0.6$ for three meshes defined with $\Theta=12^{\circ}$ and $\left(n_{x}, \theta\right)=\left(100,4^{\circ}\right),\left(200,2^{\circ}\right)$ and $\left(400,1^{\circ}\right)$. The convergence behavior of the scheme is very satisfactory. The timing, the density levels and pressure levels after the shock wave are correct. As for Sod's problem, we notice an overshoot for the internal energy and an undershoot for the density that are characteristic of the wall heating phenomena, see [23]. The Figure 14 displays the mesh at $t=0.6$ for $\Theta=90^{\circ}$ and $\left(n_{x}, \theta\right)=\left(100,2^{\circ}\right)$, one can see that the cylindrical symmetry is perfectly respected. 

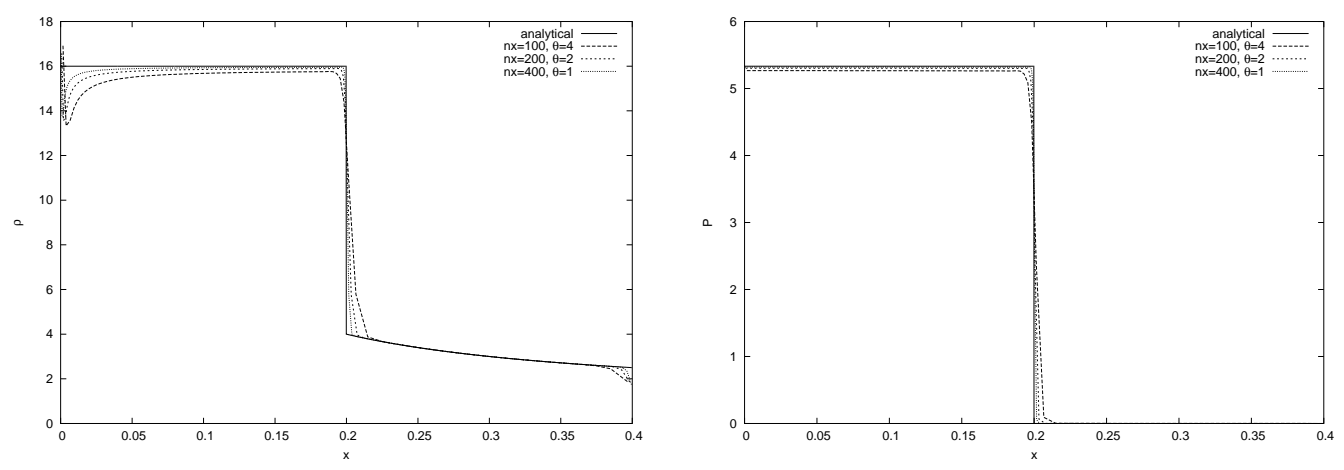

density
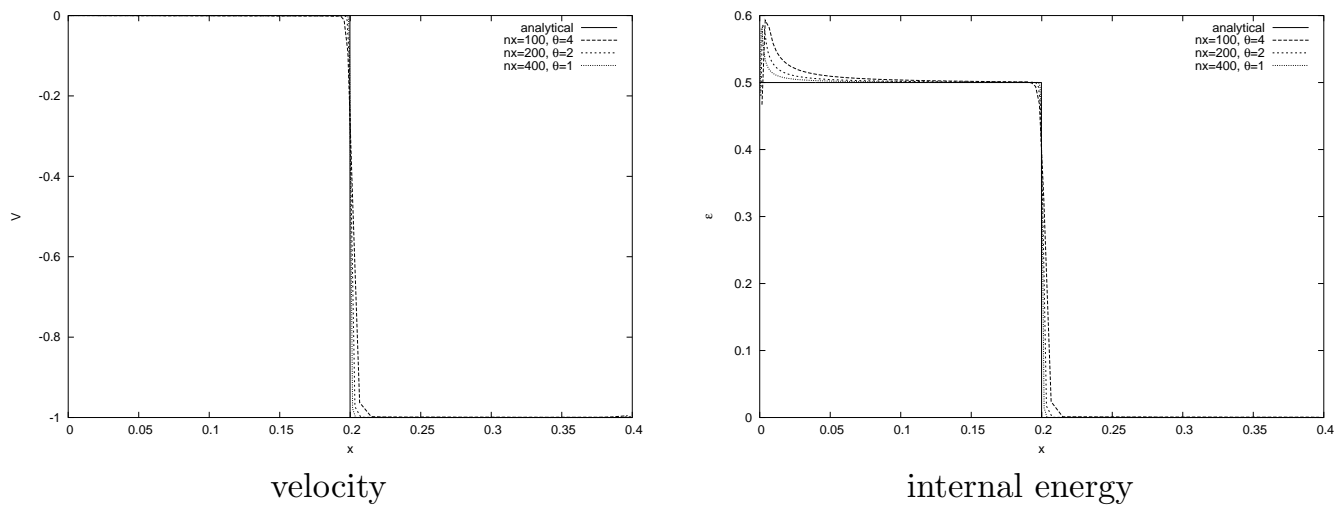

Figure 13: Cross-section for Noh's case at $t=0.6$, comparison with the analytical solution

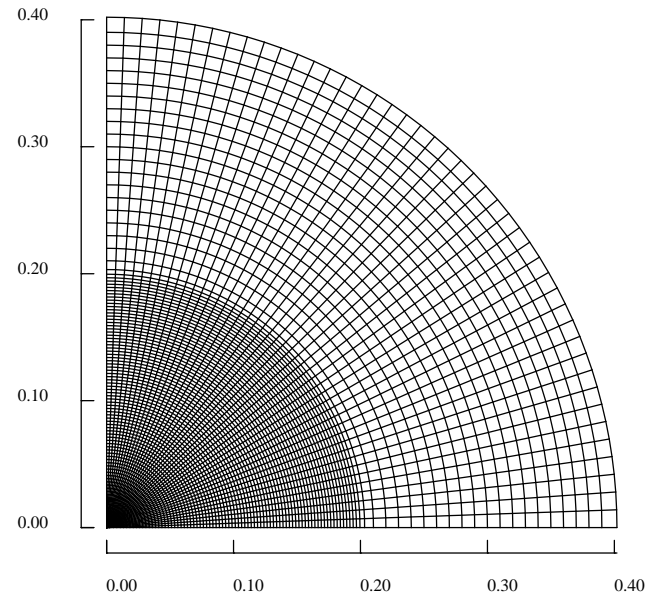

0.20

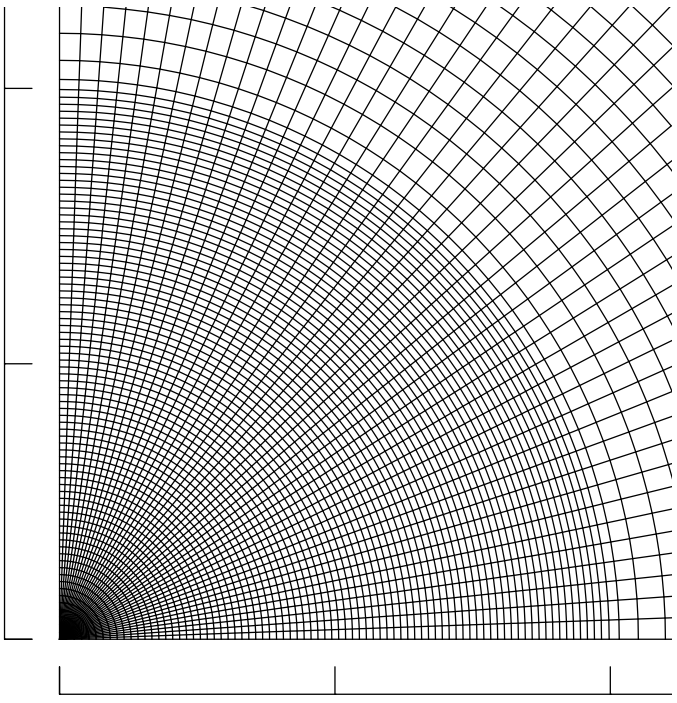

0.00
0.20

Zoom near the origin

Figure 14: Noh's case: mesh at $t=0.6$ 


\subsection{Kidder's isentropic compression}

In [17], Kidder has analytically computed the solution of the isentropic compression of a cylindrical shell filled with perfect gas. We briefly recall the main features of this solution in order to define the test case. Initially, the shell has the internal (resp. external) radius $r_{1}$ (resp. $r_{2}$ ). Let $P_{1}, P_{2}, \rho_{1}$ and $\rho_{2}$ be the pressures and densities located at $r_{1}$ and $r_{2}$. Since the compression is isentropic, we define $s=\frac{P_{2}}{\rho_{2}^{\gamma}}$, and we have $\rho_{1}=\rho_{2}\left(\frac{P_{1}}{P_{2}}\right)^{\frac{1}{\gamma}}$. The initial density and pressure profiles are given by (for $\left.r \in\left[r_{1}, r_{2}\right]\right)$ :

$$
\begin{aligned}
& \rho^{0}(r)=\left(\frac{r_{2}^{2}-r^{2}}{r_{2}^{2}-r_{1}^{2}} \rho_{1}^{\gamma-1}+\frac{r^{2}-r_{1}^{2}}{r_{2}^{2}-r_{1}^{2}} \rho_{2}^{\gamma-1}\right)^{\frac{1}{\gamma-1}}, \\
& P^{0}(r)=s\left(\rho^{0}(r)\right)^{\gamma} .
\end{aligned}
$$

The initial velocity is set to zero, $u_{0}(r)=0$. The value of $\gamma$ is set to $\gamma=1+\frac{2}{\nu}$ with $\nu=1,2,3$ whether we have a planar, cylindrical or spherical symmetry. These values are used in order to obtain a fully analytical solution. In our case we have $\nu=2$, therefore $\gamma=2$. The focusing time $\tau$ of the shell is defined (for these parameters) by

$$
\tau=\sqrt{\frac{\gamma-1}{2} \frac{r_{2}^{2}-r_{1}^{2}}{c_{2}^{2}-c_{1}^{2}}}
$$

where $c_{i}=\sqrt{s \gamma \rho_{i}^{\gamma-1}}$ for $i=1,2$ is the isentropic sound speed at $r=r_{i}$. the isentropic compression is obtained by imposing the following pressure laws at the internal and external faces of the shell

$$
\begin{aligned}
& P\left(R\left(r_{1}, t\right), t\right)=P_{1} h(t)^{-\frac{2 \gamma}{\gamma-1}} \\
& P\left(R\left(r_{2}, t\right), t\right)=P_{2} h(t)^{-\frac{2 \gamma}{\gamma-1}}
\end{aligned}
$$

Let $R(r, t)$ be the Eulerian radius (i.e. $R=\sqrt{X^{2}+Y^{2}}$ ) at $t>0$ of a fluid particle initially located on the circle of radius $r$. Looking for a solution of the form $R(r, t)=h(t) r$, we get

$$
h(t)=\sqrt{1-\left(\frac{t}{\tau}\right)^{2}} .
$$

The analytical form of the physical variables at $t \in[0, \tau[$ are:

$$
\begin{aligned}
& \rho(R(r, t), t)=h(t)^{-\frac{2}{\gamma-1}} \rho^{0}\left[\frac{R(r, t)}{h(t)}\right], \\
& u(R(r, t), t)=\frac{d}{d t} h(t) \frac{R(r, t)}{h(t)}, \\
& P(R(r, t), t)=h(t)^{-\frac{2 \gamma}{\gamma-1}} P^{0}\left[\frac{R(r, t)}{h(t)}\right] .
\end{aligned}
$$

In this test case, we have used the parameters $r_{1}=0.9, r_{2}=1, P_{1}=0.1, P_{2}=10$ and $\rho_{2}=10^{-2}$. From this, we get $\rho_{1}=10^{-3}, s=10^{5}$ and $\tau=7.26510^{-3}$. The computational domain consists of the quarter of an annulus, see Figure 15. Once more, we impose wall boundary conditions on the axis $X=0, Y=0$ and the previous pressure laws on the internal and external faces of the domain. The simulations are run on regular polar meshes defined by $\left(n_{x}, n_{y}\right)=(25,44) ;(50,88) ;(100,176)$. The computations are run up 


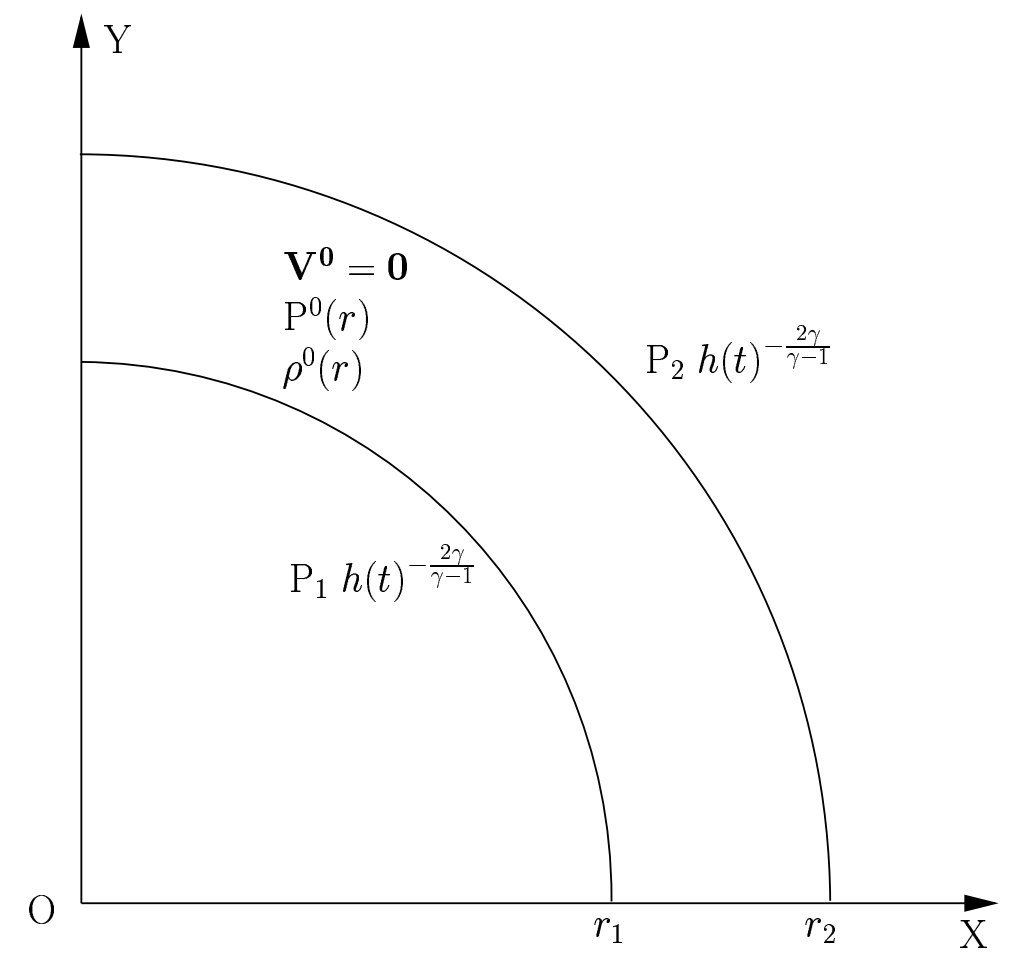

Figure 15: Initial conditions for Kidder's isentropic compression

to a final time which is very close to the focusing time, i.e. $t=0.99 \tau$. In all what follows, the time is adimensionalized by the focusing time $\tau$.

The Figure 16, where the variation of external radius of the shell is plotted, shows the excellent agreement with the analytical solution. The agreement is good for the average density $\bar{\rho}(t)^{2}$ near the focusing point, however the numerical values are smaller than the exact ones. There exists a gap between numerical and analytical density profiles at the final time. This gap gets smaller and smaller with mesh refinement, thus we believe it is a consequence of the numerical diffusion. This observation is confirmed by the profile of the entropy parameter $\alpha_{e}=\frac{P}{s \rho^{\gamma}}$. It indicates the entropy production of the scheme, and we have $\alpha_{e}=1$ in the analytical case. The gap between the exact solution and the numerical one is large on the internal face of the shell when the mesh is coarse, it also gets smaller with mesh refinement. The large entropy production of the scheme degrades the simulated compression, this explains the shape of the density profile on the internal face of the shell. The mesh at the final time $t=0.99 \tau$ is represented on Figure 17. We observe that the implosion preserves the cylindrical symmetry, even at $t=0.99 \tau$.

\footnotetext{
${ }^{2} \bar{\rho}(t)$ is evaluated from the analytical formula $\bar{\rho}(t)=0.5\left(\rho_{1}+\rho_{2}\right) h(t)^{-2}$.
} 


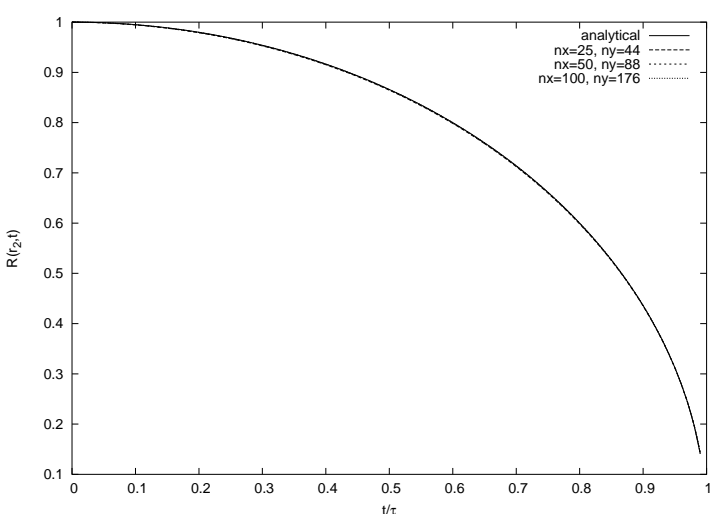

Time evolution of the external radius

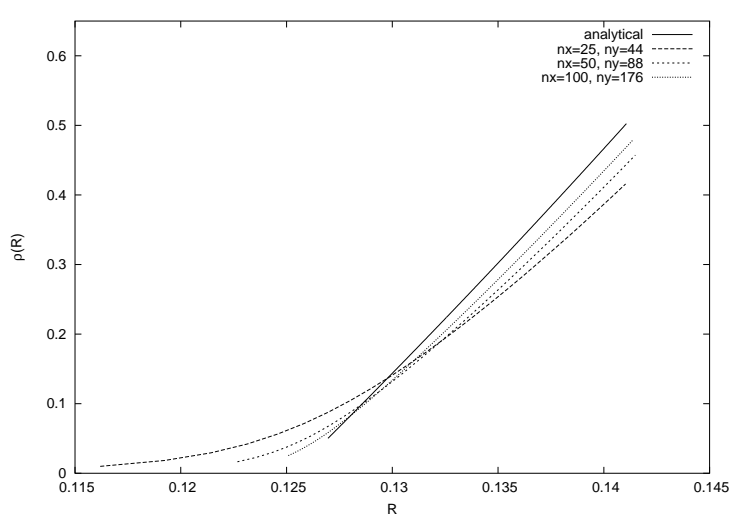

Density at $t=0.99 \tau$

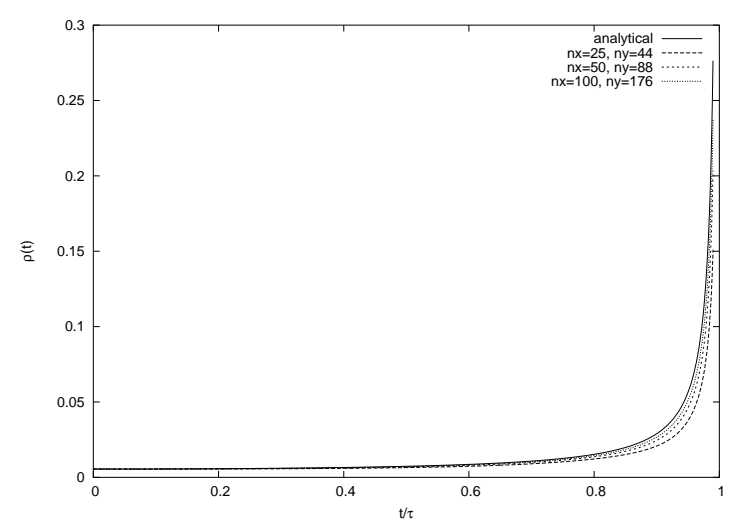

Time evolution of the average density

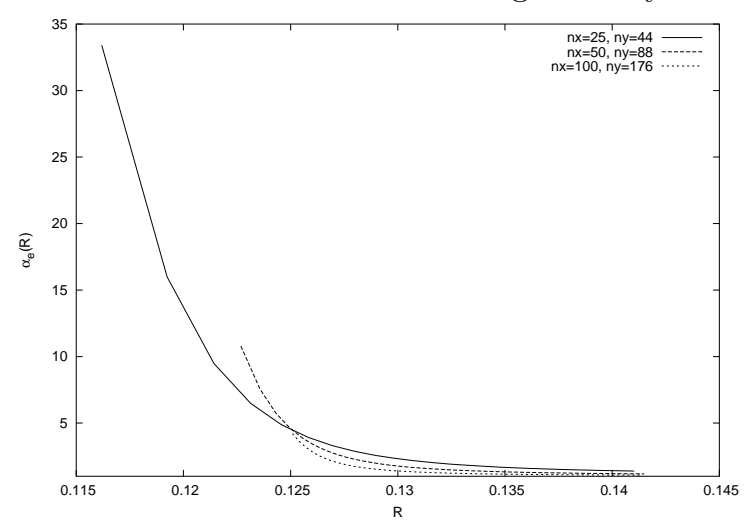

Entropy parameter at $t=0.99 \tau$

Figure 16: Kidder's isentropic compression, comparison with the analytical solution

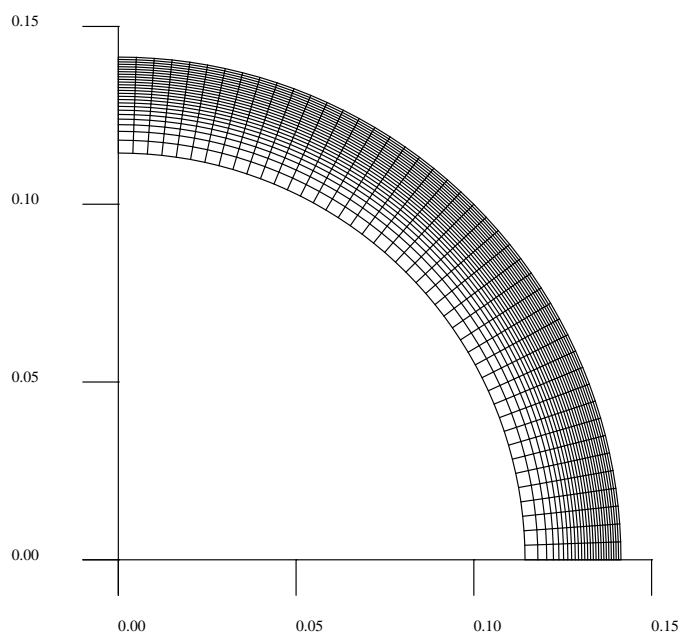

Figure 17: Kidder's isentropic compression: mesh at $t=0.99 \tau$ 


\subsection{Saltzman's shock tube}

We consider now the movement of a planar shock wave on a cartesian grid that has been stretched [10]. This is a well known difficult test case that enables to validate the robustness of our scheme when the mesh is not aligned with the fluid flow. The computational domain is the rectangle $(x, y) \in[0,1] \times[0,0.1]$. The initial mesh (shown on Figure 18) is obtained by transforming a uniform $100 \times 10$ cells with the mapping

$$
\begin{aligned}
& x_{s t r}=x+(0.1-y) \sin (x \pi), \\
& y_{s t r}=y
\end{aligned}
$$

For the material we use the equation of state of a monoatomic gas $(\gamma=5 / 3)$. The initial state is $\left(\rho^{0}, P^{0}, \boldsymbol{V}^{\mathbf{0}}\right)=(1,0,0)$. The boundary condition at $x=0$ is a normal velocity $V^{\star}=-1$ (inflow velocity). On all the other boundary, we set up wall conditions.



Figure 18: Saltzman test case: initial mesh

The exact solution is a planar shock wave that moves at speed $D=4 / 3$ from left to right. The propagation of the shock wave at $t=0.6$ is displayed in Figure 19. The important result is that our scheme preserves one-dimensional solution very well (except for the first and last layer in the vertical direction, this is an effect of up and down wall boundary conditions). The Figure 19 shows the mesh at $t=0.75$ when the shock wave hits the right boundary $(x=1)$. Behind the shock wave, the initial mesh is distorted, all the horizontal line stays almost parallel with respect to the other ones. The density profile at $t=0.6$ is displayed on Figure 21 . The shock level is not uniform, but it oscillates around the exact value $\rho=4$.

The robustness of our scheme is clearly demonstrated by this test case: we are able to reach time $t=0.93$ which corresponds to two successive rebounds of the shock wave on the vertical boundaries of the domain, see Figure 22. After this time, the computation stops because the mesh become tangled.

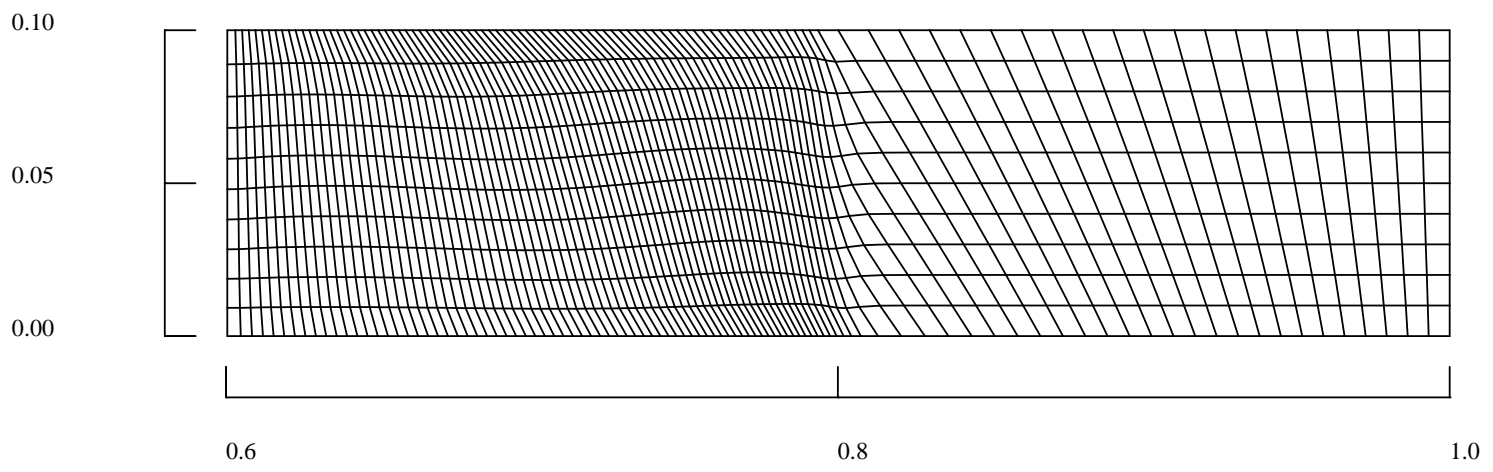

Figure 19: Saltzman's test: mesh at $t=0.6$ 


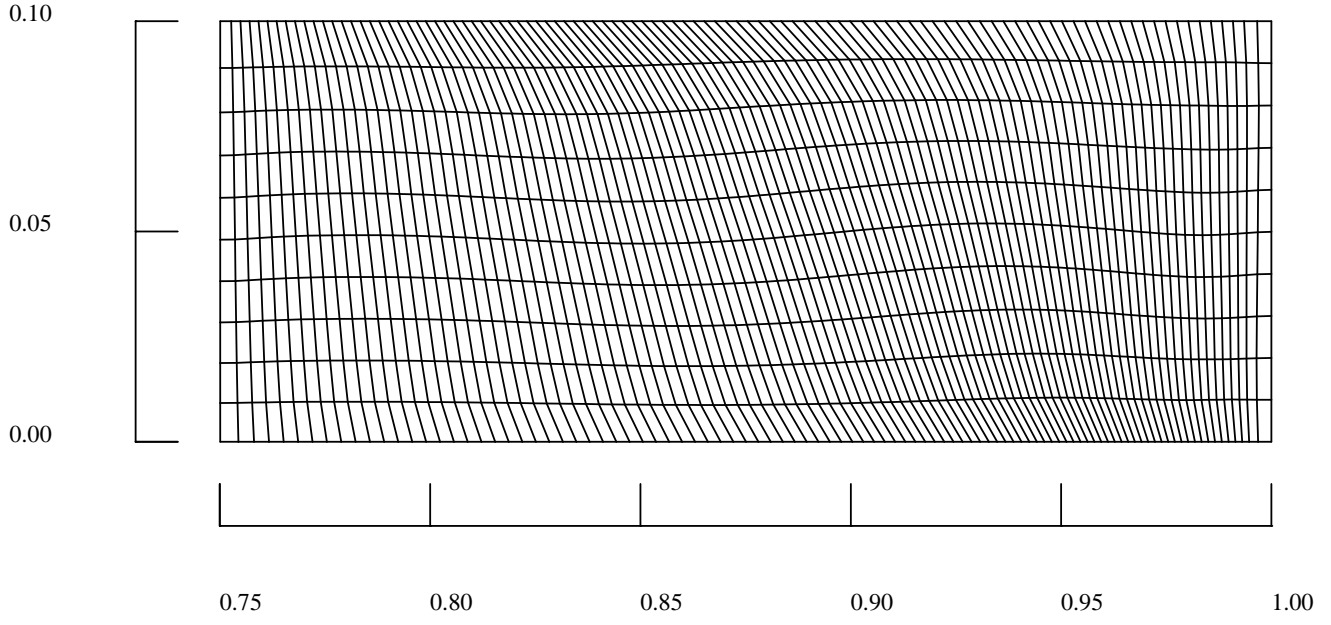

Figure 20: Saltzman's test: mesh at $t=0.75$

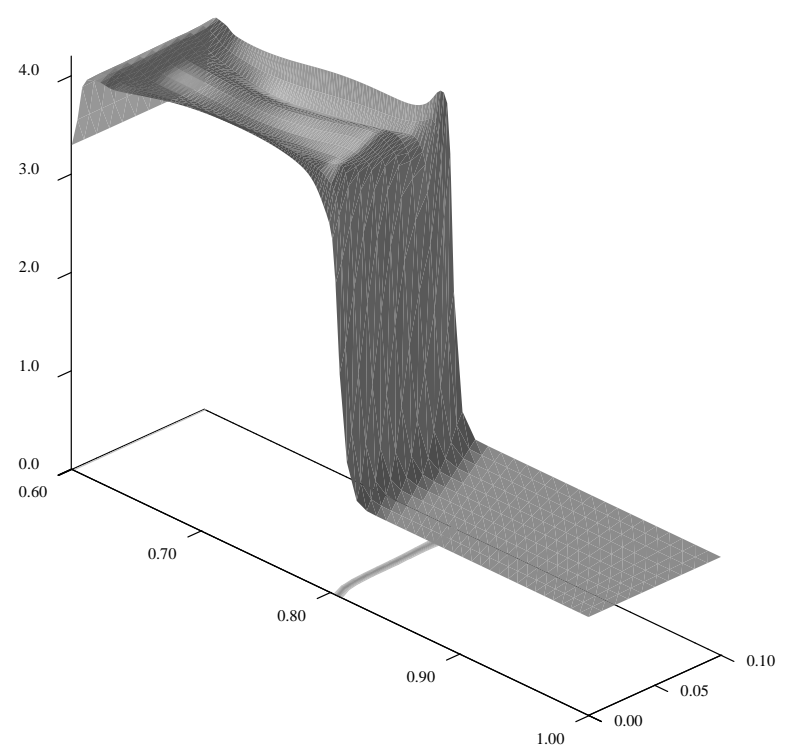

Figure 21: Saltzman's test: density profile at $t=0.6$ 


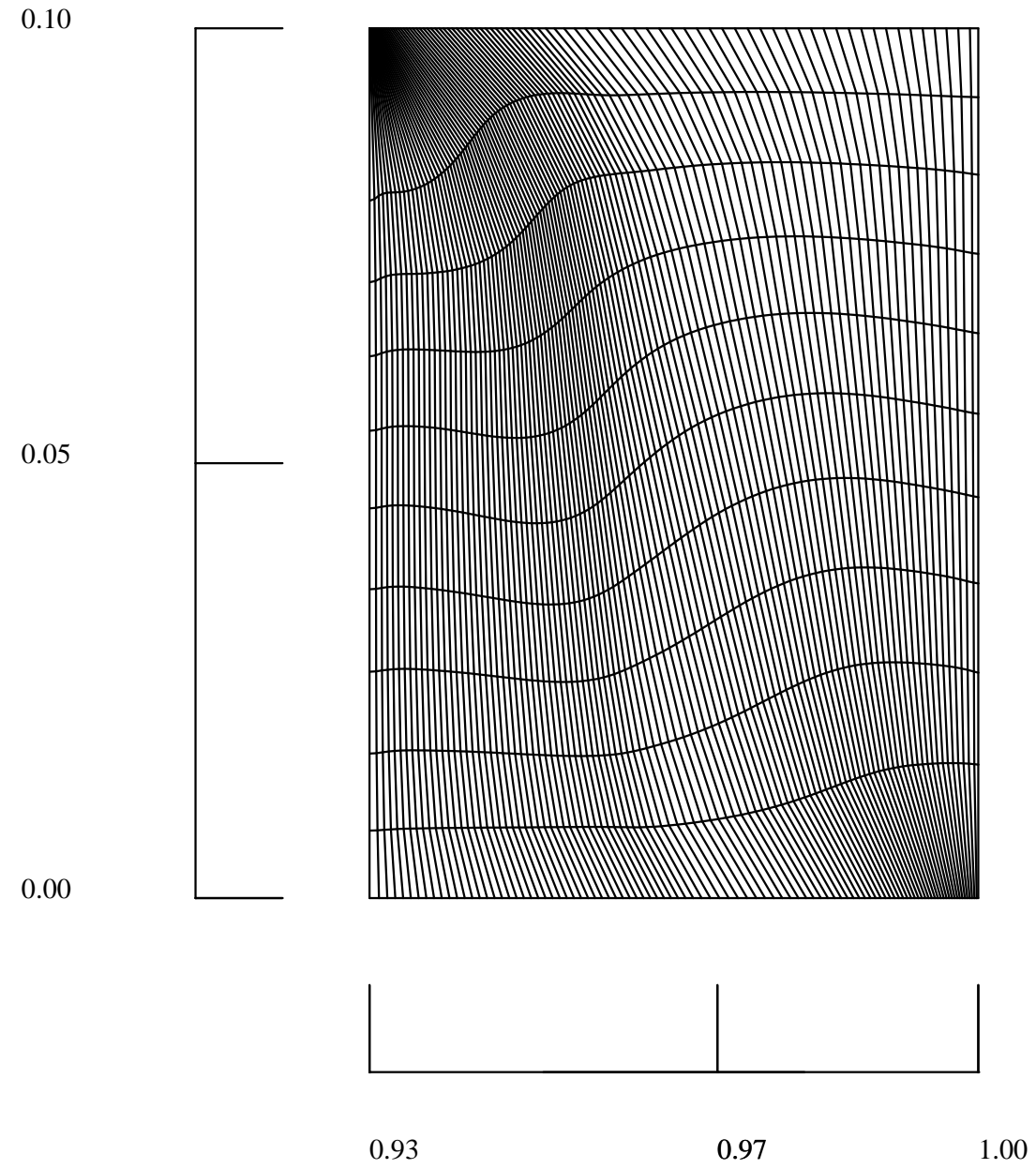

Figure 22: Saltzman's test: mesh at $t=0.93$ 


\subsection{Sedov's test case}

We consider the propagation of a high intensity cylindrical shock wave generated by a strong explosion, see for instance [30]. Each boundary conditions are wall conditions. The gas of interest is monoatomic $\left(\gamma=\frac{5}{3}\right)$. It is initially at rest $\left(\rho^{0}, P^{0}, \boldsymbol{V}^{\mathbf{0}}\right)=(1,0,0)$ and we set up an energy spike at the center. The simulation is done first on a Cartesian grid and then on a polar grid. In order to compare the results, the same amount of energy is added for each computations.

On the cartesian grids, the computational domain is $(x, y) \in[0,1] \times[0,1]$. In the case of the coarsest mesh (i.e. $\left.\left(n_{x}, n_{y}\right)=(50,50)\right)$, the internal specific energy of the first cell is set to $\epsilon^{0}=5000$. This corresponds to sub-domain $(x, y) \in[0,1 / 50] \times[0,1 / 50]$. The finer meshes are $\left(n_{x}, n_{y}\right)=(100,100)$ and $\left(n_{x}, n_{y}\right)=(200,200)$. In order to have the same amount of energy, we also initialize with the specific internal energy $\epsilon^{0}=5000$ in $[0,1 / 50] \times[0,1 / 50]$.

In the case of the polar grids, the domain is defined by $(r, \theta) \in[0,1] \times\left[0,90^{\circ}\right]$. The mesh is made of quadrangles with triangles at the center. The parameters $\left(n_{x}, n_{y}\right)$ correspond to the number of layers in the radial direction and of angular sectors. The coarsest mesh is defined by $\left(n_{x}, n_{y}\right)=(50,25)$. We initialize the specific internal energy on triangles and define the sub-domain $(r, \theta) \in[0,1 / 50] \times\left[0,90^{\circ}\right]$. As the area to initialize is different in this case, we need to modify the internal energy so that the energy amount is the same as in the case of the Cartesian grid. Thus we choose $\epsilon^{0}=5000 \frac{4}{\pi}$. As for the Cartesian mesh, the finest polar grids are $\left(n_{x}, n_{y}\right)=(100,50)$ and $\left(n_{x}, n_{y}\right)=(200,100)$, and in both case, the cells to initialize are those in the sub-domain $(r, \theta) \in[0,1 / 50] \times\left[0,90^{\circ}\right]$.

The Figure 23 represents the final mesh at $t=0.1$ obtained for the two types of meshes. Clearly, our initialization leads to the same results. However, we notice that on the cartesian grid non-convex cells appear but this does not pose any problem to the nodal solver.
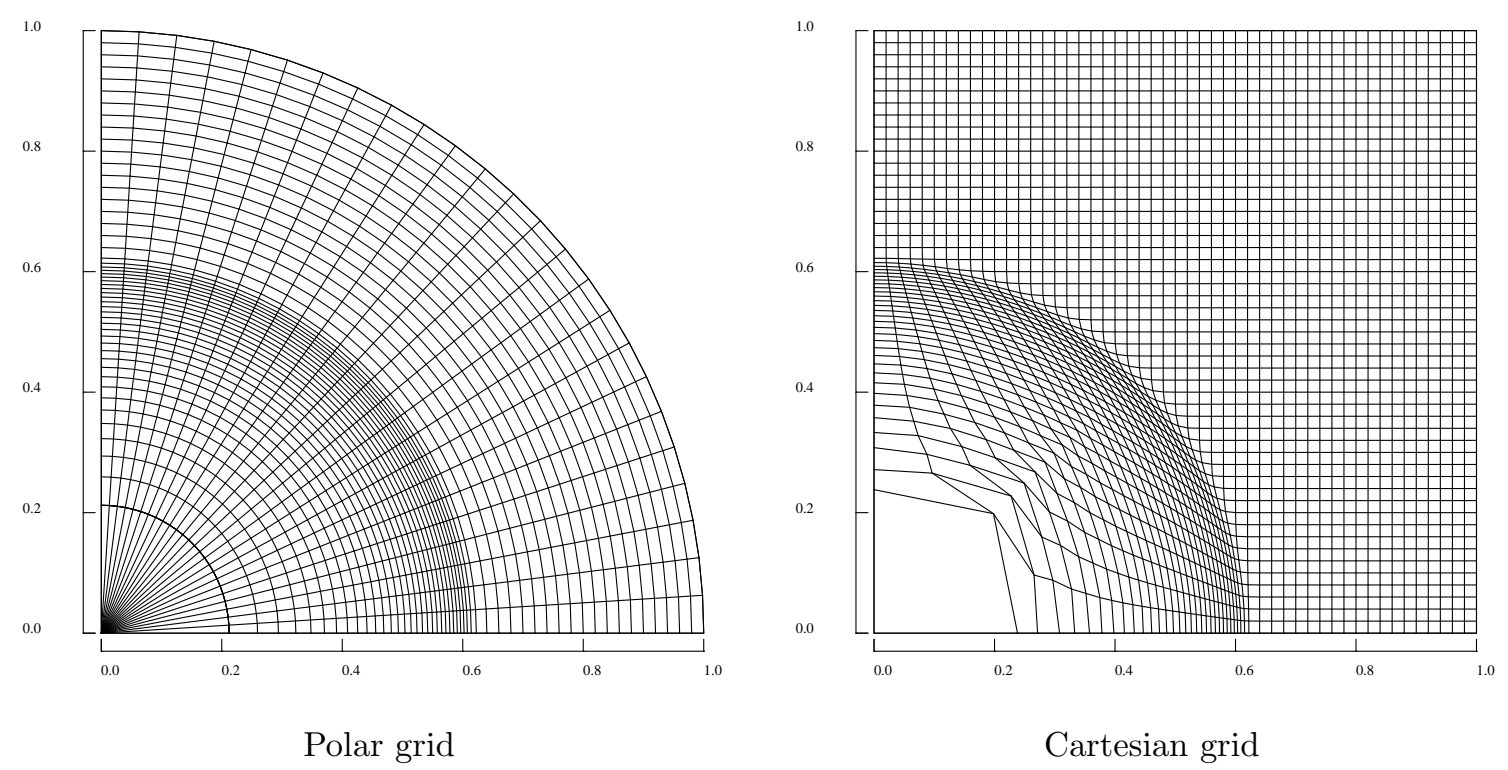

Figure 23: Sedov's test case: mesh at $t=0.1$ for the polar and Cartesian meshes

The comparison of the density profiles for the two types of grids is done on Figures 24,27 and 26 . We notice that for the polar grid, the cylindrical symmetry of the flow is perfectly preserved, see Figures 23 and 24. The symmetry is also well preserved on Cartesian grids, as shown in Figure 25 on the left, the density profile on the diagonal are slightly different from those on the $x$ - and $y$ - axis (which are identical). In Figure 25 on the right are represented the density values in all the cells in function of the centroid radius. We also 
observe that the symmetry is well preserved. Consequently, these results on Cartesian grids show the ability of our new scheme to provide good results even when the mesh is not aligned with the flow. Moreover, the Figures 27 and 26 demonstrates that a mesh convergence study for both grids leads to similar results.

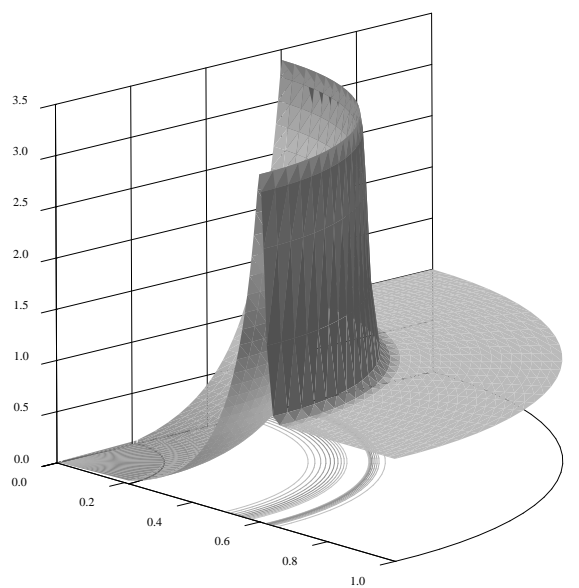

Polar grid

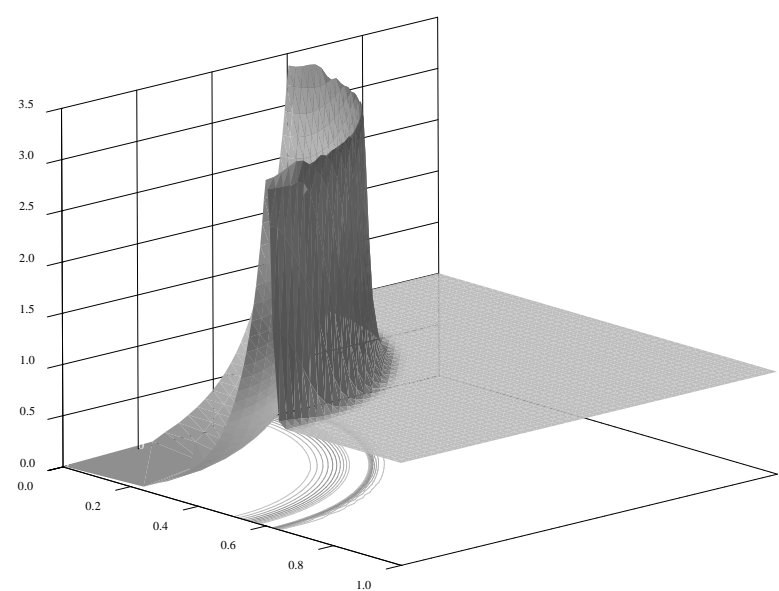

Cartesian grid

Figure 24: Sedov's test case: density level at $t=0.1$



Density profiles along different axis

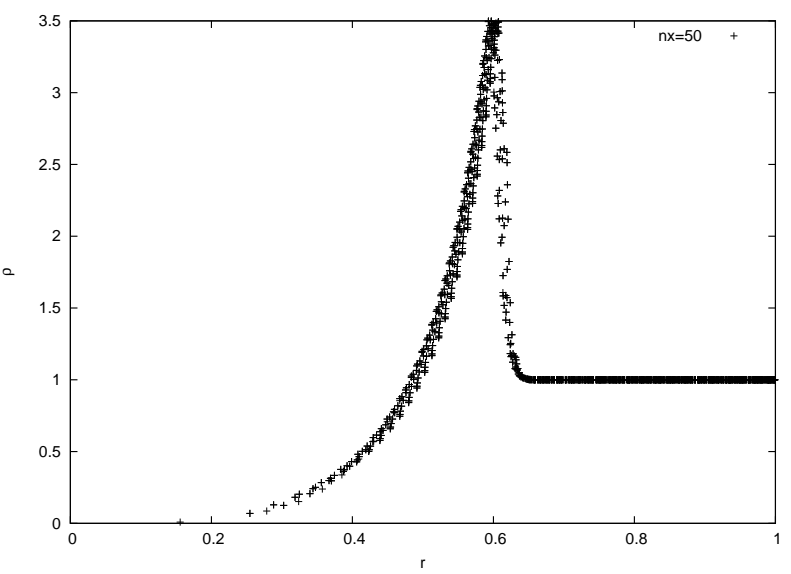

Density in all the cells

Figure 25: Sedov's test case on Cartesian grid at $t=0.1$ 


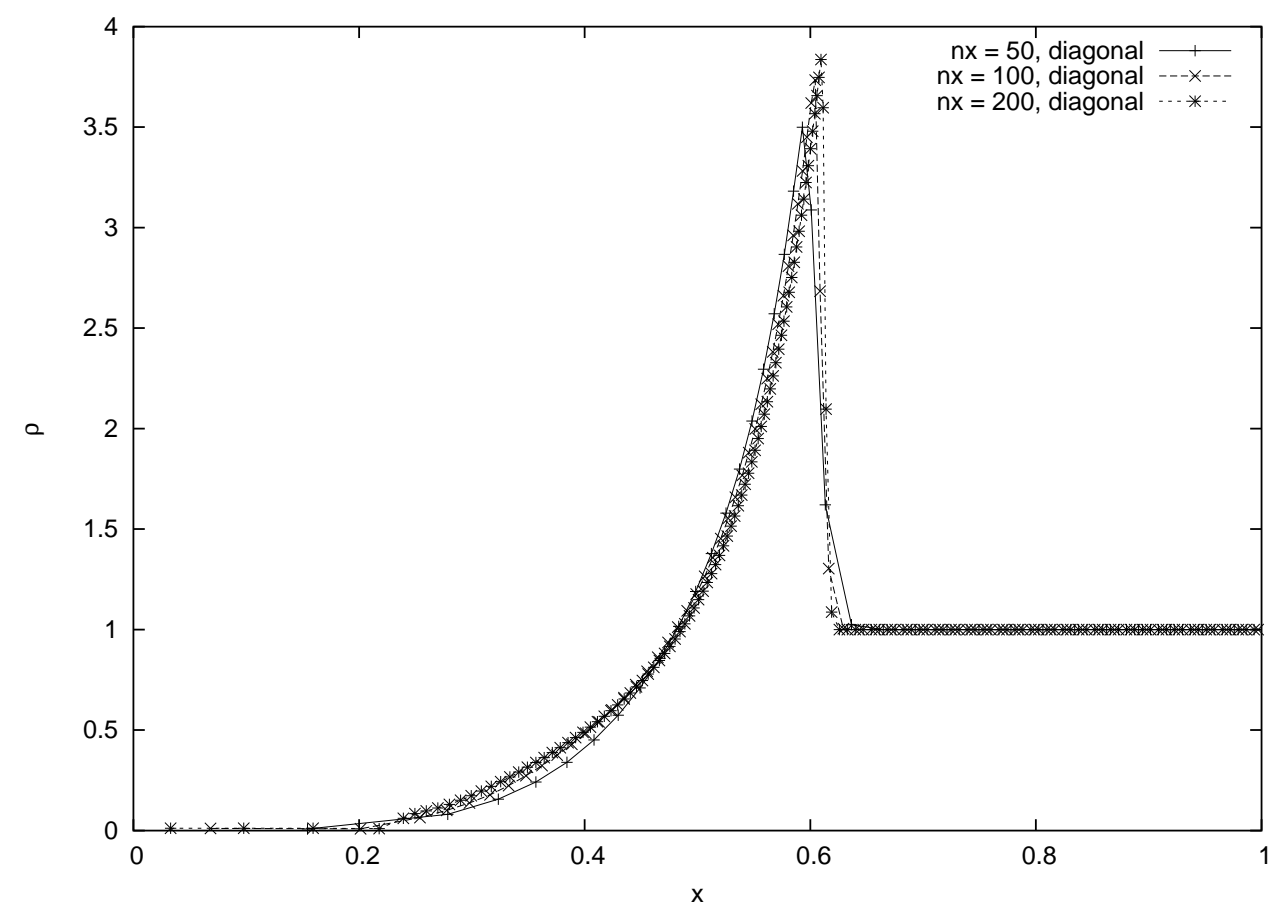

Figure 26: Sedov's test case: cartesian grid convergence for density profiles at $t=0.1$

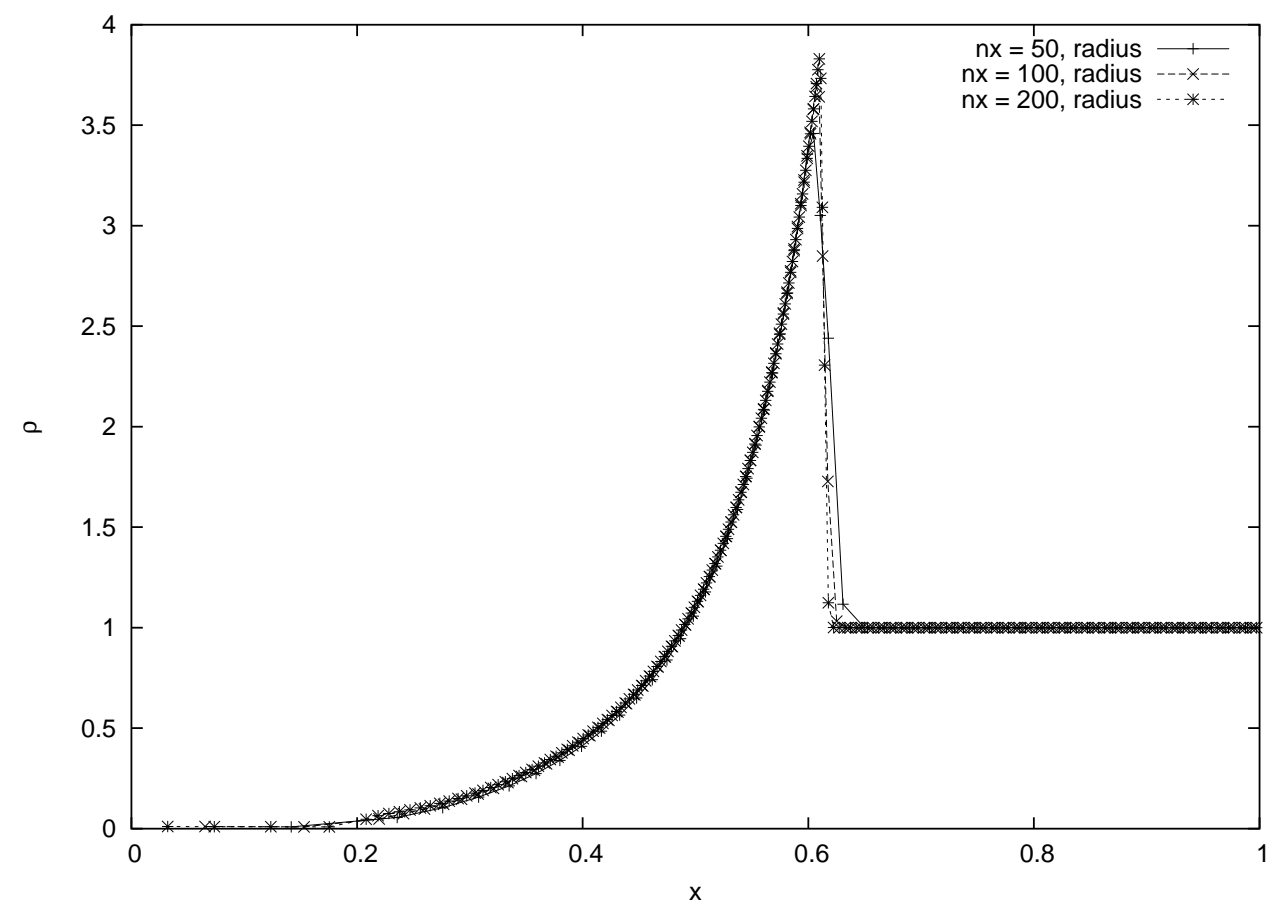

Figure 27: Sedov's test case: polar grid convergence for density profiles at $t=0.1$ 


\subsection{Perturbation of the isentropic Kidder's compression}

In this paragraph, we give an original test case representative of the field of hydrodynamic instabilities. The aim of this test is to show the ability of our new scheme to reproduce the growth of such instabilities. In order to define the test, we consider the isentropic implosion of a cylindrical shell as in section 6.3. We initially perturb the internal and external faces of the shell, see Figure 15. Then, we study the time evolution of the perturbations. An approximate analytical solution is available for the perturbation amplification in the linear regime. This solution is obtained with the technique developed in [13]. We briefly recall the main results about the analytical solution in order to set up the test case. More details can be found in [22] and $[3]$.

First we define the mesh perturbation. We are given a Cartesian frame $(0, x, y)$ and polar coordinates $(r, \theta)$. The polar coordinates of the vertices are given by

$$
\begin{aligned}
& r^{p}=r+a_{0} \xi_{r}(r, \theta), \\
& \theta^{p}=\theta+a_{0} \xi_{\theta}(r, \theta),
\end{aligned}
$$

where $a_{0}$ is the amplitude of the initial perturbation. The vector $\boldsymbol{\xi}=\left(\begin{array}{l}\xi_{r} \\ \xi_{\theta}\end{array}\right)$ is defined by

$$
\begin{aligned}
& \xi_{r}(r, \theta)=n\left(A_{1} r^{-n-1}+A_{2} r^{n-1}\right) \cos (n \theta), \\
& \xi_{\theta}(r, \theta)=-n\left(A_{1} r^{-n-1}+A_{2} r^{n-1}\right) \sin (n \theta) .
\end{aligned}
$$

The integer $n$ is the perturbation mode and $A_{1}, A_{2}$ are constants which are determined by the perturbations $\xi_{1}$ and $\xi_{2}$ given at the internal and external interfaces, i.e. $\xi_{1}=\xi_{r}\left(r_{1}, 0\right)$ and $\xi_{2}=\xi_{r}\left(r_{2}, 0\right)$. We have

$$
\left\{\begin{array}{l}
A_{1}=-\frac{1}{n} \frac{\xi_{1} r_{2}^{n-1}-\xi_{2} r_{1}^{n-1}}{r_{1}^{-n-1} r_{2}^{n-1}-r_{1}^{n-1} r_{2}^{-n-1}}, \\
A_{2}=-\frac{1}{n} \frac{\xi_{1} r_{2}^{-n-1}-\xi_{2} r_{1}^{-n-1}}{r_{1}^{-n-1} r_{2}^{n-1}-r_{1}^{n-1} r_{2}^{-n-1}} .
\end{array}\right.
$$

The analytical form (56) is justified by the fact that $\boldsymbol{\xi}$ derives from a potential. We check that $\nabla \cdot \boldsymbol{\xi}=0$, that is the perturbation is incompressible. We have a divergence free perturbation.

Using the previous results, we get the form of coordinates of the perturbed mesh

$$
\begin{aligned}
& x^{p}=\left(1+\frac{a_{0}}{r} \xi_{r}\right)\left[x \cos \left(a_{0} \xi_{\theta}\right)-y \sin \left(a_{0} \xi_{\theta}\right)\right], \\
& y^{p}=\left(1+\frac{a_{0}}{r} \xi_{r}\right)\left[x \sin \left(a_{0} \xi_{\theta}\right)+y \cos \left(a_{0} \xi_{\theta}\right)\right] .
\end{aligned}
$$

This is illustrated by Figure 28 where is represented the perturbed mesh for the half annulus of radii $r_{1}=0.9, r_{2}=1$, with the following data: perturbation mode $n=8$, internal and external levels $\xi_{1}=0$, $\xi_{2}=1$, amplitude $a_{0}=0.02$. In [22], we show that the amplification factor $a(\theta, t)$ of the perturbation at the external interface is given by:

$$
a(\theta, t)=n\left[A_{1} r^{-n-1} G_{1}(t)+A_{2} r^{n-1} G_{2}(t)\right] \cos (n \theta),
$$

where $t \in\left[0,1\left[\right.\right.$ is the time ${ }^{3}$. The functions $G_{1}$ and $G_{2}$ are:

$$
\begin{aligned}
& G_{1}(t)=\sqrt{1-t^{2}} \cos \left[\frac{\sqrt{n}}{2} \log \left(\frac{1-t}{1+t}\right)\right], \\
& G_{2}(t)=\frac{1}{2} \sqrt{1-t^{2}}\left[\left(\frac{1-t}{1+t}\right)^{\sqrt{n} / 2}+\left(\frac{1+t}{1-t}\right)^{\sqrt{n} / 2}\right] .
\end{aligned}
$$

\footnotetext{
${ }^{3}$ the time is adimensionalized by the focusing time $\tau$.
} 


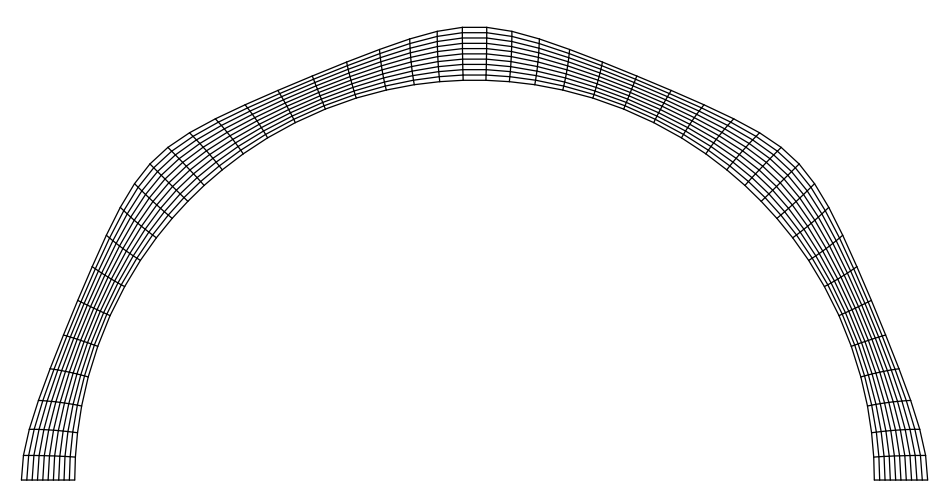

Figure 28: Perturbed mesh

Next, we present the results for the following cases: modes 4 and 8 with a perturbation of the external interface and mode 8 with a perturbation of the internal and external interfaces. For a given mode, we first define a mesh compatible with this mode, i.e. with enough angular sectors per wave length. Then, we compute the non perturbed implosion and store the location of the vertex of the external face that corresponds to $\theta=0$, namely $R\left(r_{2}, t\right)$. Then we perturb the mesh according to (57). An implosion simulation is done on this perturbed mesh. Then we store $R_{p}\left(r_{2}, t\right)$, the location of the same vertex. The amplification factor is defined by the ratio

$$
a_{\text {num }}(t)=\frac{R^{p}\left(r_{2}, t\right)-R\left(r_{2}, t\right)}{a_{0}} .
$$

In each of the perturbed simulations, the initial amplitude is set to $a_{0}=10^{-6}$ in order to stay in the linear phase. The final time of each simulations is $t=0.99$. The time step follows 55 .

6.6.1 Mode $n=4, \xi_{1}=0, \xi_{2}=1, a_{0}=10^{-6}$

The computational domain is defined using the symmetries of mode 4 , i.e. $(r, \theta) \in\left[r_{1}, r_{2}\right] \times\left[0, \frac{\pi}{2}\right]$. We use equi-angular meshes defined by $\left(n_{x}, n_{y}\right)=(25,44),\left(n_{x}, n_{y}\right)=(50,88)$ and $\left(n_{x}, n_{y}\right)=(100,176)$.

In Figure 30, we show the results for the coarsest mesh. The time evolution of the amplitude for the vertices defined by $\theta=0, \pi / 8, \pi / 4,3 \pi / 8, \pi / 2$ (see Figure 29) are stored. We see that the expected symmetries for the vertices $\theta=0, \pi / 4, \pi / 2$ are perfectly respected. Moreover the amplification factor for the vertices defined by $\theta=\pi / 8,3 \pi / 8$ is almost null, but we notice a very small amplification, of the order of a percent, when we are near the focusing. On Figure 31, we plot the time evolution of the perturbation obtained numerically for the three meshes and the analytical solution. The comparison is satisfactory since the numerical amplification is coherent with the analytical one. This comparison is even more satisfactory as the scheme is only first order in time and space.

We present on Figure 32 the mesh that corresponds to a perturbed simulation near the focusing time. The initial mesh is defined by $\left(n_{x}, n_{y}\right)=(25,176)$. The initial amplitude is set to $a_{0}=10^{-3}$, so that, at the final time, we are in the non linear regime. 


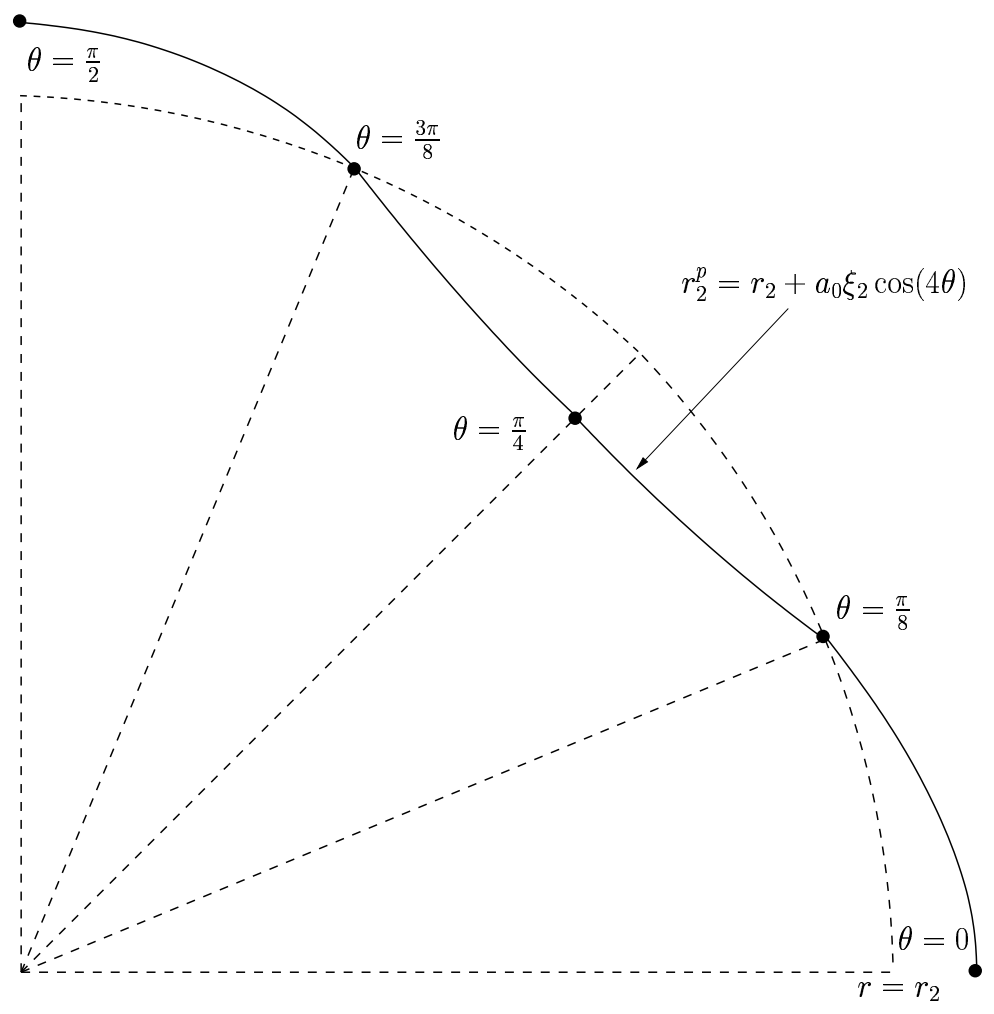

Figure 29: Mode $n=4$, location of the vertices of interest

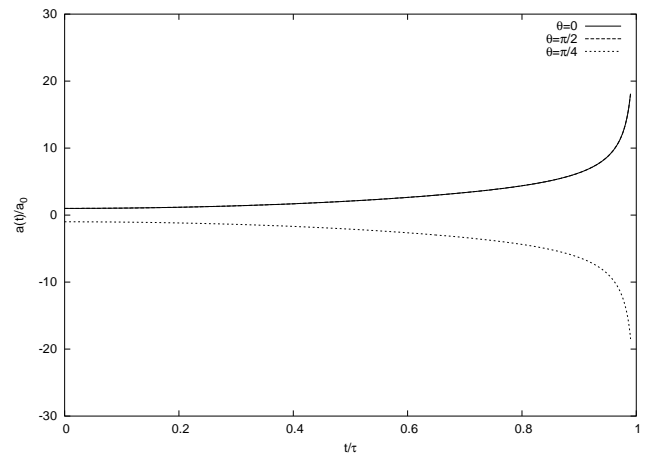

Points of maximal amplitude



Points of vanishing amplitude

Figure 30: Mode $n=4$, time evolution of the external perturbation for several vertices of interest 




Figure 31: Mode $n=4$, time evolution of the amplitude of the external perturbation

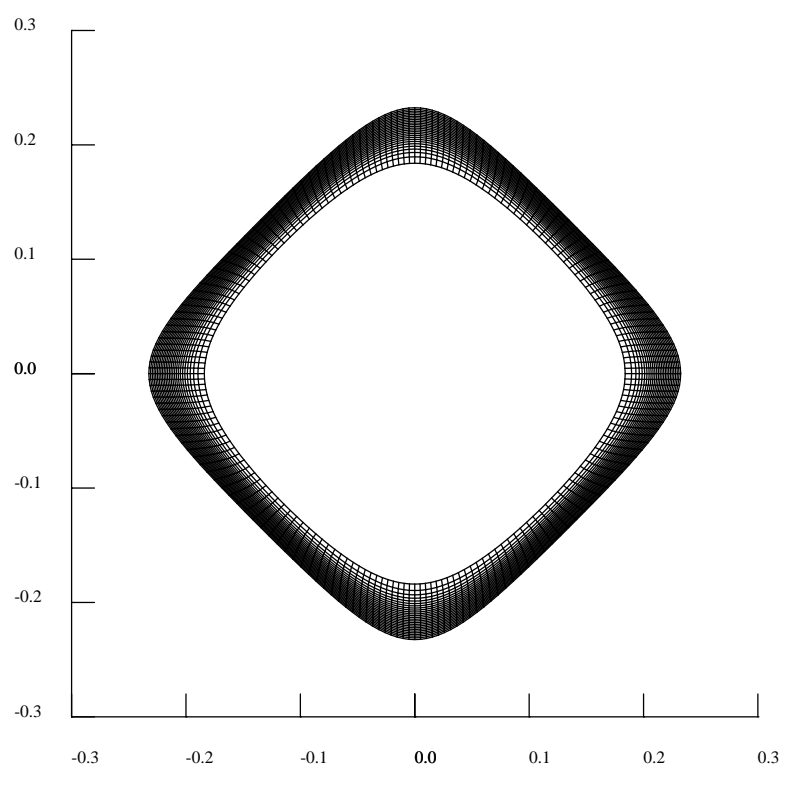

Mesh at $t / \tau=0.98$

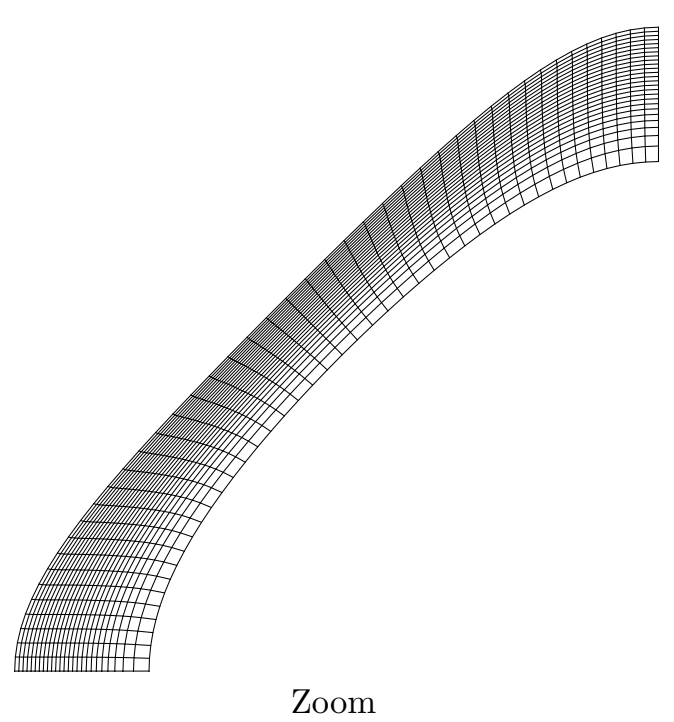

Figure 32: Mode $n=4$, non-linear phase $a_{0}=10^{-3}$ 
6.6.2 Mode $n=8, \xi_{1}=0, \xi_{2}=1, a_{0}=10^{-6}$

The computational domain is defined using the symmetries of the mode 8 , i.e. $(r, \theta) \in\left[r_{1}, r_{2}\right] \times\left[0, \frac{\pi}{2}\right]$. We use equi-angular meshes with $\left(n_{x}, n_{y}\right)=(25,44),\left(n_{x}, n_{y}\right)=(50,88)$ and $\left(n_{x}, n_{y}\right)=(100,176)$. Figure 33

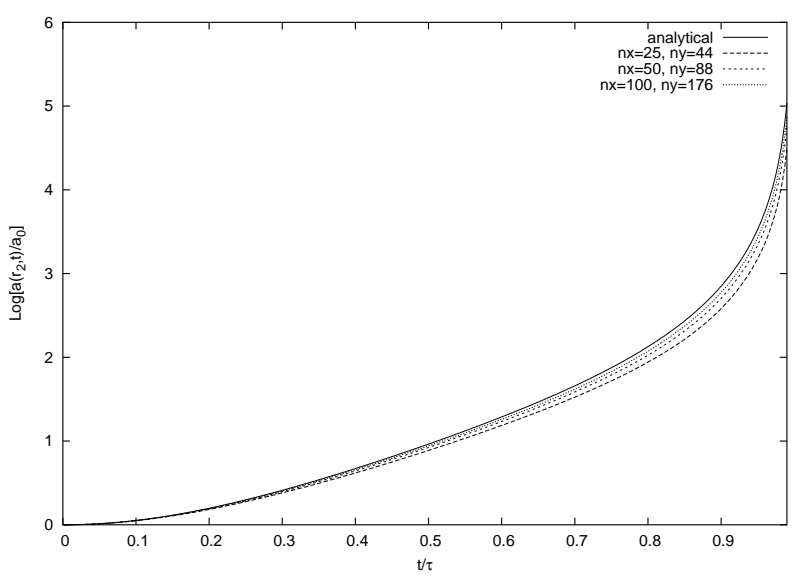

Figure 33: Mode $n=8$, time evolution of the amplitude of the external perturbation

shows a good agreement with the analytical solution for the time evolution of the amplitude of the external perturbation. As for the $n=4$ mode case, the scheme converges by lower values.

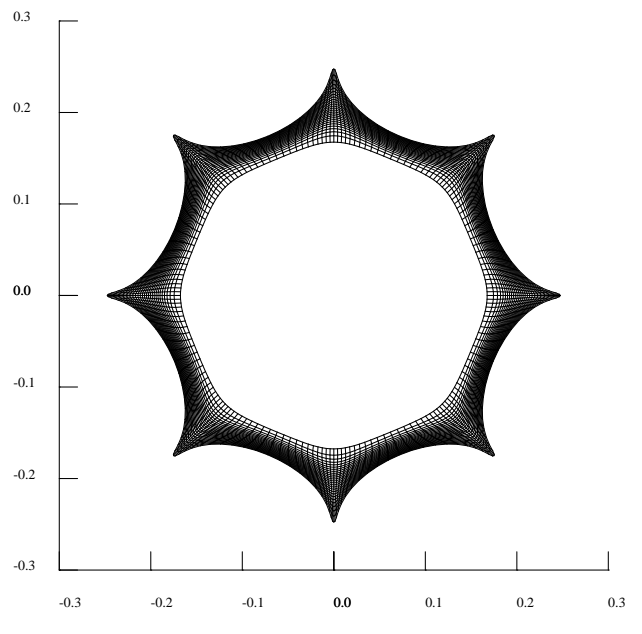

Mesh at $t / \tau=0.98$

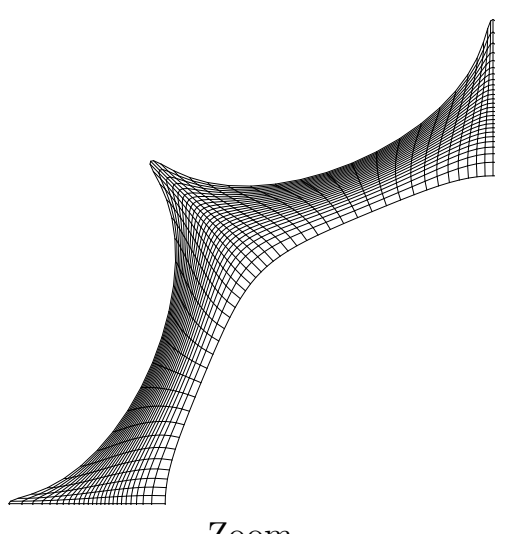

Zoom

Figure 34: Mode $n=8$, non linear phase for $a_{0}=10^{-3}$

On Figure 34 we display the mesh obtained during the non-linear phase for the flow perturbed on the full geometry. The initial mesh is defined by $\left(n_{x}, n_{y}\right)=(25,176)$. The initial amplitude of the perturbation is $a_{0}=10^{-3}$. 
6.6.3 Mode $n=8, \xi_{1}=2.565293, \xi_{2}=1, a_{0}=10^{-6}$

In this case, we have introduced a non zero initial perturbation on the internal interface. The level of $\xi_{1}$ is defined so that at the final time $t=0.99$, the amplitude of the perturbation on the external face comes back to its initial value. This permits to undergo the coupling of the perturbations between the two interfaces. This coupling enables to get a periodic growth of the perturbation on the internal face. In this case, we use the same meshes and the same domain as in the previous case.



Figure 35: Coupled mode $n=8$, growth of the perturbation on the internal interface

The results are very satisfactory, see Figure 35 . They show the ability of our scheme to reproduce the coupling of perturbations between the internal and external faces. 


\subsection{ICF-like test case}

In this paragraph, we present a simplified test case of a cylindrical ICF target. The target is made of two materials which have the same perfect gas equation of state $(\gamma=5 / 3)$. The data are chosen to be representative of ICF situations. The interface between the gas and the shell is located at $r_{1}=0.1$ and the external radius of the target is $r_{2}=0.11$, see Figure 36 . The initial state of the gas is given by $\left(\rho_{1}, P_{1}, \boldsymbol{V}_{\mathbf{1}}\right)=\left(0.01,2.510^{9}, 0\right)$ and the state inside the shell is $\left(\rho_{2}, P_{2}, \boldsymbol{V}_{\mathbf{2}}\right)=\left(1,2.510^{11}, 0\right)$. On the external surface of the shell, we apply a pressure law $P^{\star}=10^{14}\left(1+a_{0} \sin (2 \theta)\right)$. We study this target in two configurations: the first is monodimensional with $a_{0}=0$ and the second is bidimensional with $a_{0}=0.2$. This second configuration may represent crudely the hydrodynamic part of an ICF flow which results from an inhomogeneous irradiation of the target.

For the monodimensional problem the mesh is made of 25 identical angular sectors on $\left[0,90^{\circ}\right]$ and 200 layers (100 uniform layers in the gas and 100 layers with a geometrical progression in the shell). A geometrical progression is applied so that we have a good representation of the contact discontinuity between the two materials (we have the same mass in the two cells around the interface). The time evolution of internal interface has been displayed in Figure 37. This shows the two acceleration periods at $t=110^{-9}$ and $t=310^{-9}$ of the interface and at the end the deceleration of the interface until the stagnation time at $t=510^{-9}$. In order to validate our results for the monodimensional configuration, we compare them to the results obtained with a monodimensional code named CHIVAS. This code uses a Von Neumann-Richtmyer type scheme, see [16]. In Figure 37, we compare the trajectory of the internal interface with our scheme and with CHIVAS. The two curves can be almost superimposed, this demonstrates the quality of our scheme.

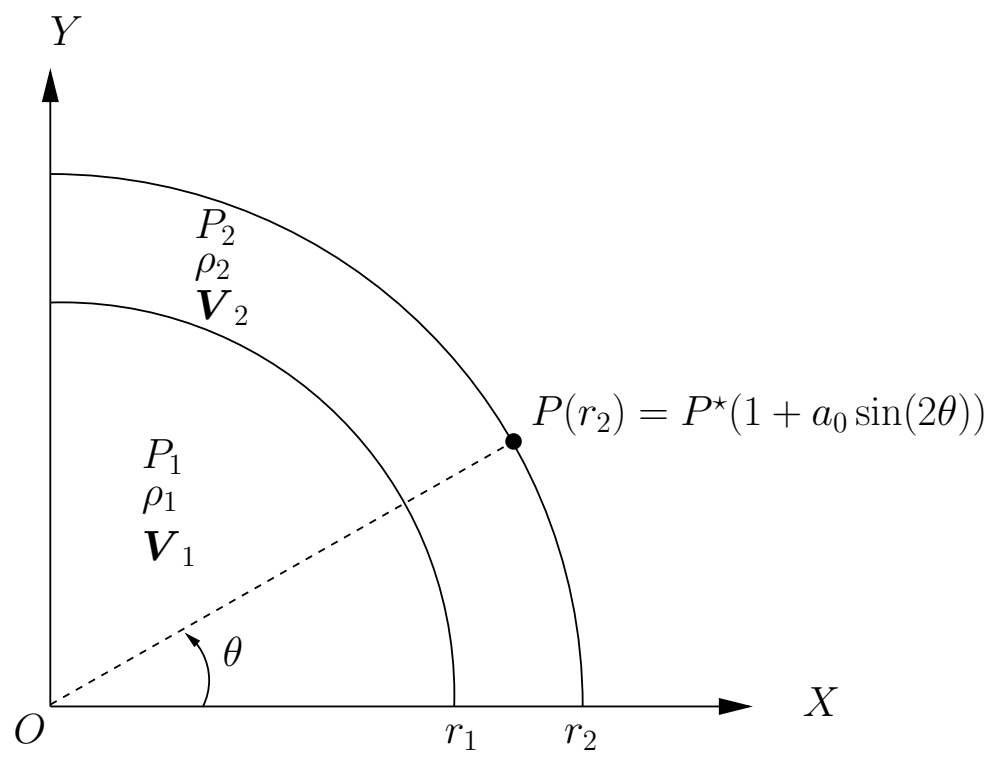

Figure 36: ICF-like target: initial conditions

For the bidimensional case we use two types of meshes. One is a polar mesh (see Figure 38) with 40 angular sectors on $\left[0,90^{\circ}\right]$ and 100 layers (50 uniform layers in the gas and 50 layers with a geometrical progression in the shell). The other one is a multibloc mesh in which we have a square in the central part of the target and nearly the same mesh as the polar grid for the rest of the domain (Figure 38). The Figure 39 represents the mesh at $t=4.410^{-9}$ for both configurations. The non-uniformity of the pressure on the shell boundary leads to a bidimensional deformation of the internal interface.

The Figure 40 illustrates one of the advantage to use a multibloc mesh. We can see that the time step in the case of polar mesh reduce to very small value $\left(\approx 110^{-14}\right)$ whereas for the multibloc mesh we keep reasonable time step $\left(\approx 110^{-13}\right)$. The very small value of the time step for the polar mesh is due to the very 
small size of the triangular cells located at the center. Moreover the multibloc mesh makes the computation more robust and enables to deal with higher non-uniformity value for $a_{0}$.



Figure 37: Monodimensional ICF-like test case: time evolution of the internal interface radius



Polar mesh

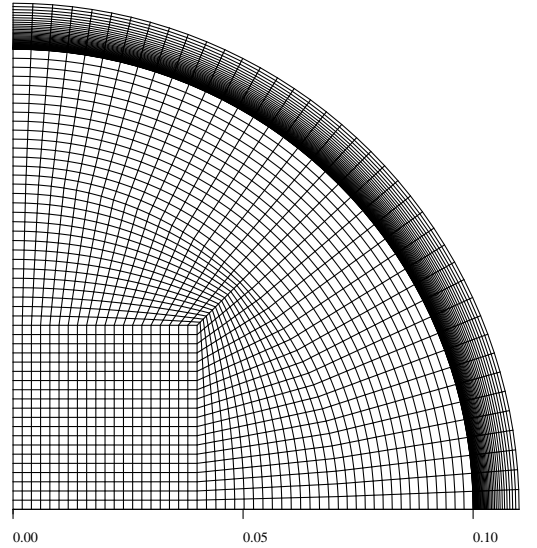

Multibloc mesh

Figure 38: Initial mesh for bidimensional ICF-like test case 


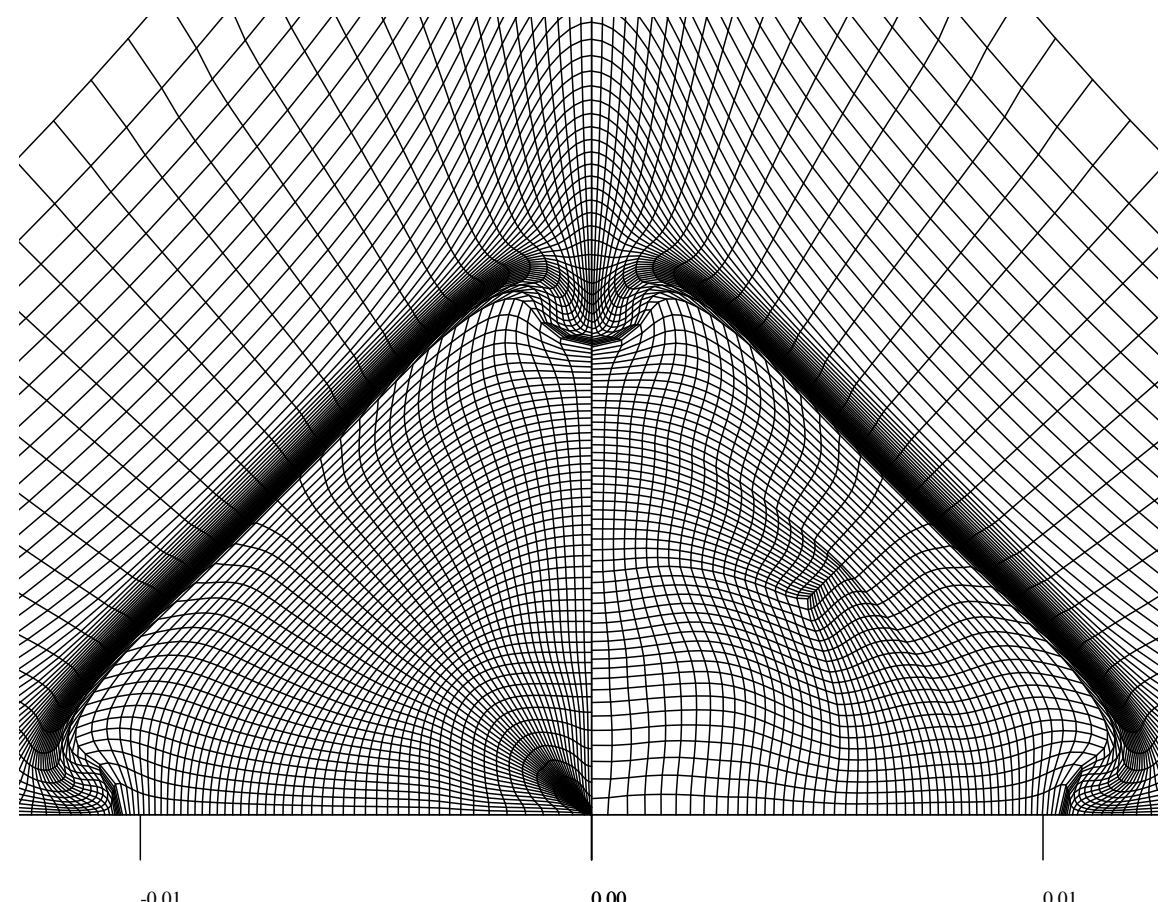

Figure 39: Result for two type of meshes at time $t=4.410^{-9}$

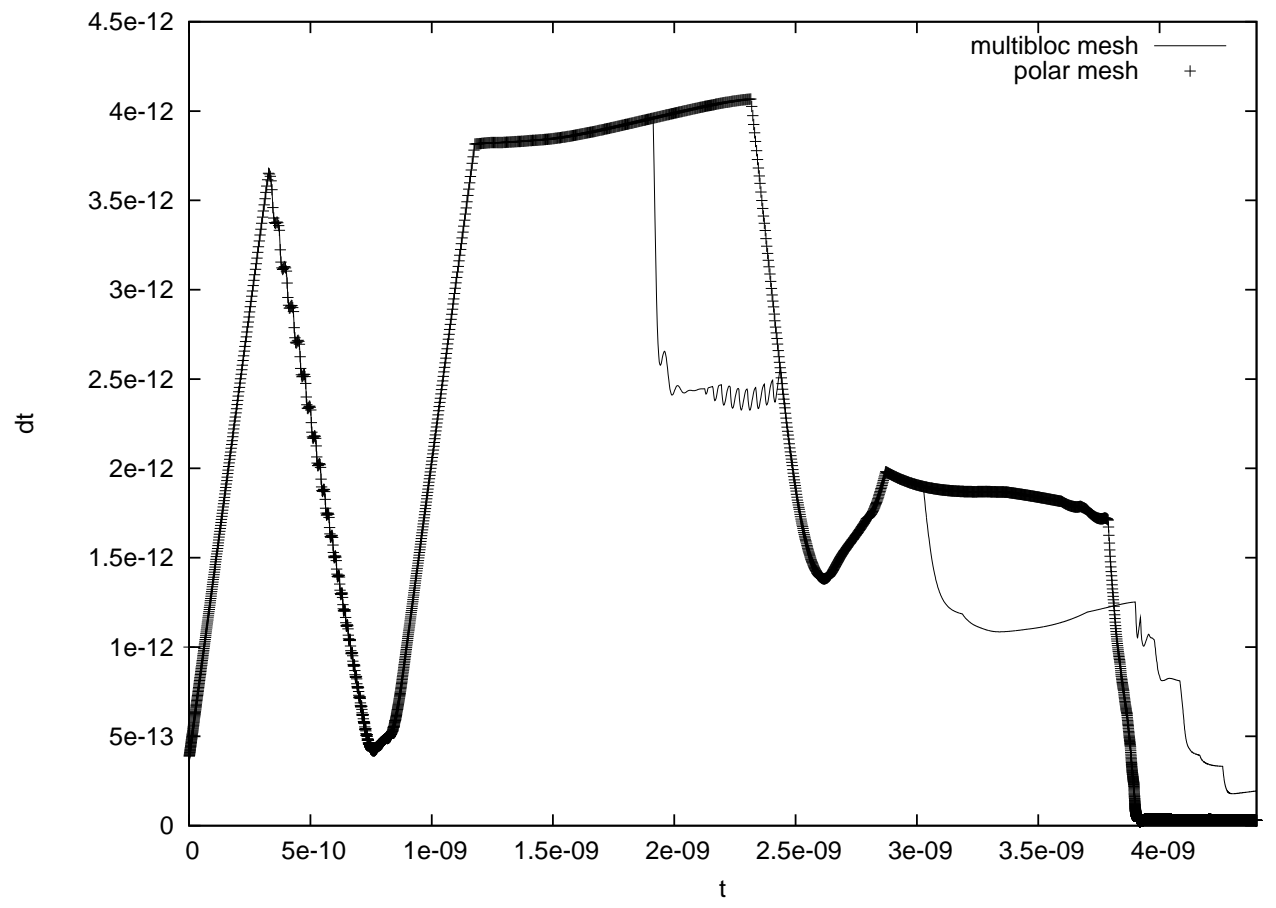

Figure 40: Time step during the simulations 


\section{Conclusion and perspectives}

We have developed a new Lagrangian cell-centered conservative scheme. This scheme relies on a genuinely two-dimensional nodal solver. It can be interpreted as the two-dimensional generalization of the Godunov acoustic solver. The main new features of the algorithm is the introduction of four pressures on each edge, two for each node on each side of the edge. This extra degree of freedom allows us to construct a nodal solver that fulfills two properties. First, the conservation of momentum and total energy is ensured. Second, a semi-discrete entropy inequality is provided. The face fluxes and the nodal velocities, are all evaluated in a coherent manner. We have checked that, in the case of one-dimensional problems (with planar or cylindrical symmetry), our solver is exactly equivalent to the one-dimensional Godunov acoustic solver.

The scheme is only first order accurate, however it appears to be quite robust and versatile according to the numerical results obtained for the various test cases presented in this paper.

The next step is to develop the axisymmetric extension of our scheme. Then, in order to improve the accuracy of the scheme we will study its second order extension probably with a MUSCL type method. This new Lagrangian method looks promising both as a stand-alone entity but also as a foundation for a more general method which may include ALE and/or AMR techniques. The uniform cell centering of the solution variables provides a consistent basis for employing a wide range of well proven remapping and adaptation schemes.

\section{References}

[1] R. Abgrall, R. Loubère, and J. Ovadia. A Lagrangian Discontinuous Galerkin-type method on unstructured meshes to solve hydrodynamics problems. Int. J. Numer. Meth. Fluids, 44:645-663, 2004.

[2] D. J. Benson. Computational methods in Lagrangian and Eulerian hydrocodes. Comp. Meth. in Appl. Mech. and Eng., 99:235-394, 1992.

[3] J. Breil, L. Hallo, P.-H. Maire, and M. Olazabal-Loumé. Hydrodynamic instabilities in axisymmetric geometry self-similar models and numerical simulations. Laser Part. Beams, 23:155-160, 2005.

[4] J.C. Campbell and M.J. Shashov. A tensor artificial viscosity using a mimetic finite difference algorithm. J. Comput. Phys., 172:739-765, 2001.

[5] E. J. Caramana, M. J. Shashkov, and P. P. Whalen. Formulations of Artificial Viscosity for Multidimensional Shock Wave Computations. J. Comput. Phys., 144:70-97, 1998.

[6] E.J. Caramana, D.E. Burton, M.J. Shashov, and P.P. Whalen. The construction of compatible hydrodynamics algorithms utilizing conservation of total energy. J. Comput. Phys., 146:227-262, 1998.

[7] E.J. Caramana and M.J. Shashkov. Elimination of artificial grid distorsion and hourglass-type motions by means of Lagrangian subzonal masses and pressures. J. Comput. Phys., 142:521-561, 1998.

[8] A. Chorin and J. Marsden. A Mathematical Introduction to Fluid Mechanics. Springer Verlag, 1992.

[9] B. Després and C. Mazeran. Lagrangian gas dynamics in two dimensions and lagrangian systems. Arch. Rational Mech. Anal., 178:327-372, 2005.

[10] J. K. Dukowicz and B. Meltz. Vorticity errors in multidimensional Lagrangian codes. J. Comput. Phys., 99:115-134, 1992.

[11] J.K. Dukowicz, M.C. Cline, and F.S. Addessio. A general topology method. J. Comput. Phys., 82:29-63, 1989.

[12] S.K. Godunov, A. Zabrodine, M. Ivanov, A. Kraiko, and G. Prokopov. Résolution numérique des problèmes multidimensionnels de la dynamique des gaz. Mir, 1979. 
[13] S.J. Han and B. R. Suydam. Hydrodynamic instabilities in an imploding cylindrical plasma shell. Physical Review A, 26:926-939, 1982.

[14] C.W. Hirt, A. Amsden, and J.L. Cook. An arbitrary Lagrangian-Eulerian computing method for all flow speeds. J. Comput. Phys., 14:227-253, 1974.

[15] W. H. Hui, P. Y. Li, and Z. W. Li. A Unified Coordinate System for Solving the Two-Dimensional Euler Equations. J. Comput. Phys., 153:596-637, 1999.

[16] S. Jacquemot and A. Decoster. Z scaling of collisional ne-like x-ray lasers using exploding foils. refraction effects. Laser Part. Beams, 9:517-526, 1991.

[17] R. E. Kidder. Laser-driven compression of hollow shells : power requirements ans stability limitations. Nuclear Fusion, 1:3-14, 1976.

[18] L. Landau and E. Lifchitz. Mécanique des Fluides. Mir, 1989.

[19] J. D. Lindl. Inertial Confinement Fusion. Springer, 1998.

[20] R. Loubère. Une méthode particulaire Lagrangienne de type Galerkin discontinu. Application à la mécanique des fluides et à l'interaction laser plasma. PhD thesis, Université Bordeaux I, 2002.

[21] R. Loubère and M.J. Shashkov. A subcell remapping method on staggered polygonal grids for arbitrarylagrangian-eulerian methods. J. Comput. Phys., 23:155-160, 2004.

[22] P.-H. Maire, J. Breil, L. Hallo, and M. Olazabal-Loumé. Hydrodynamic instabilities in cylindrical geometry self-similar models and numerical simulations. In 31st EPS Conference on Plasma Physics, London, 2004.

[23] W. F. Noh. Errors for calculations of strong shocks using artificial viscosity and an artificial heat flux. J. Comput. Phys., 72:78-120, 1987.

[24] R. D. Richtmyer and K. W. Morton. Difference methods for initial-value problems. John Wiley, 1967.

[25] Y. Saillard. Hydrodynamique de l'implosion d'une cible FCI. C. R. Acad. Sci. Paris Série IV, 1:705-718, 2000 .

[26] G. A. Sod. A survey of several finite difference methods for systems of non-linear hyperbolic conservation laws. J. Comput. Phys., 27:1-31, 1978.

[27] J. von Neumann and R. D. Richtmyer. A method for the numerical calculations of hydrodynamical shocks. J. Appl. Phys., 21:232-238, 1950.

[28] P. Whalen. Algebraic limitations on two dimensionnal hydrodynamics simulations. J. Comput. Phys., 124:46-54, 1996.

[29] M. L. Wilkins. Calculation of elastic plastic flow. Methods in Computationnal Physics, 3, 1964.

[30] Y. B. Zel'dovich and Y. P. Raizer. Physics of Shock Waves and High-Temperature Hydrodynamic Phenomena, vol I. Academic Press, 1966. 